SERVIÇO DE PÓS-GRADUAÇÃO DO ICMC-USP

Data de Depósito: 19/02/2004

Assinatura: Af tra Paule jam paio fre /ones

\title{
Invariantes de germes do plano no plano ${ }^{1}$
}

\author{
Mariana Rodrigues da Silveira
}

Orientador: Profa. Dra. Roberta Godoi Wik Atique

Dissertação apresentada ao Instituto de Ciências Matemáticas e de Computação da Universidade de São Paulo, como parte dos requisitos para obtenção do título de Mestre em Ciências - Área: Matemática.

\section{USP - São Carlos}

Fevereiro/2004

\footnotetext{
${ }^{1}$ Este trabalho teve suporte financeiro da Fapesp proc: 01/12532-0
} 


\section{A Comissão Julgadora:}

Profa. Dra. Roberta Godoi Wik Atique

Rowill

Profa. Dra. Maria Aparecida Soares Ruas

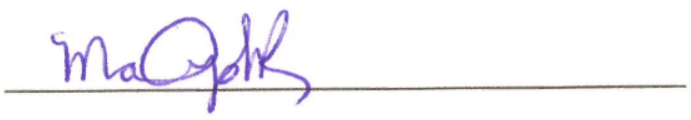

Profa. Dra. Angela Maria Sitta

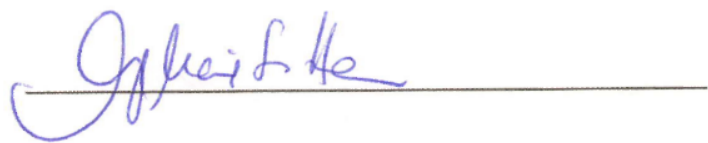


Aos meus pais 


\section{Agradecimentos}

A Deus, meu eterno companheiro, que esteve comigo e me iluminou em todos os momentos, não deixando que eu desanimasse nos momentos difíceis e dividindo comigo os bons momentos.

A Prof. Roberta pelo incentivo, pelas sugestões e críticas, pela paciência, comprenssão, dedicação o confiança.

Aos meus pais João Batista e Maria Hermínia, por me darem a vida, por serem pessoas maravilhosas nas quais me espelhei para criar meus valores e me tormar a pessoa que hoje sou, por me darem todo o apoio moral e financeiro e por me orientarem e me encorajarcm em todos os momentos da minha vida. Minha cterna gratidão!

As minhas irmãs Marina e Joana, aos meus primos, tios e avós, que estiveram ao meu lado me apoiando e incentivando em todos os momentos. Ao meu avô Zezinho, meu exemplo de vida, de bondade, honestidade e força de vontade. Vô, queria que vc estivesse aqui!

Especialmente à minha tia Lúcia por todas as suas orações pelo meu succsso. Obrigada, tia Lu!

Aos meus amigos, aqueles que me acompanharam e me ajudaram de perto e aqueles que torceram por mim de longe, meus mais profundos agradecimentos. A minha turma Alex, Aline, Fábio, Grazielle, Ronaldinho, Flávia, Luci Any, Kolly e Érica pelo companheirismo, pelas conversas, pelos churrascos, pelo apoio e carinho. Aos meus queridos amigos Camila, minha irmãzinha do coração, e Fonnando, meu anjo da guarda. Lo pessoal da minha salinha e aos meus amigos de Paraguaçu. Valeu, gente!

Aos meus professores, que me incentivaram e me oricntaram, especialmente a lres, o Biasi, o Wagner e o Ladeira.

Aos funcionários do ICMC por toda a ajuda e atcnção dispensada.

Lnfim, a todos aqueles que colaboraram de alguma forma para a realização deste trabalho.

Muito Obrigada! 


\section{Resumo}

O objetivo do trabalho é estudar os invariantes de germes de aplicações do plano no plano, que são: o número de cúspides $(c(f))$ e o número de dobras $(d(f))$ que aparecem no discriminante de uma perturbação estável do germe $f$. Além disso, mostramos que $c(f)$ e $d(f)$ são invariantes topológicos. No caso particular em que $f$ é um germe de corank 1 , encontramos fórmulas que simplificam o cálculo de $c(f)$ e $d(f)$. 


\begin{abstract}
In this work we deal with invariants for map germs from the plane to the plane. These invariants are the number of cusps $(c(f))$ and nodes $(d(f))$ that appear in the discriminant of a stable perturbation of the initial germ $f$. We show also that $c(f)$ and $d(f)$ are topological invariants. When $f$ has corank 1 we present more simple formulas for $c(f)$ and $d(f)$.
\end{abstract}




\section{Sumário}

Introdução $\quad$ ii

1 Teoria de Singularidades 1

1.1 Germes e $\mathcal{A}$-equivalência . . . . . . . . . . . . . . . . . . . . 1

1.2 Espaço Tangente . . . . . . . . . . . . . . . . . . . 3

1.3 Determinação Finita e Desdobramentos . . . . . . . . . . . . . . . . . . 9

1.4 Teorema da Preparação . . . . . . . . . . . . . . . . . . 11

1.5 Equivalência de Contato . . . . . . . . . . . . . . . . 13

2 Anéis de Cohen-Macaulay $\quad 15$

2.1 Resultados Básicos de Âlgebra Comutativa . . . . . . . . . . . . . . . . 15

2.2 Dimensão . . . . . . . . . . . . . . . . . . . . . . . 17

2.3 Grade e Anéis de Cohen-Macaulay . . . . . . . . . . . . . . . 18

2.4 Módulos Projetivos e o Teorema de Hilbert-Burch . . . . . . . . . . . . 20

3 Germes de Variedades Analiticas e Ideais de Fitting 23

3.1 Germes de Variedades Analíticas Complexas . . . . . . . . . . . . . 23

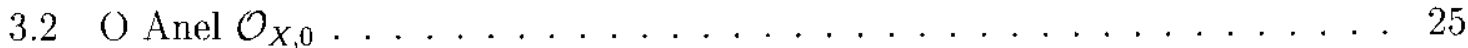

3.3 Normalização . . . . . . . . . . . . . . . . . . . 27

3.4 Intersecção Completa com Singularidade Isolada . . . . . . . . . . . . 28

3.5 Ideais de Fitting . . . . . . . . . . . . . . . . . . . . . 28

4 Cúspides e Nós de Germes de $\mathbb{C}^{2}$ em $\mathbb{C}^{2} \quad 30$

4.10 invariante delta $(\delta) \ldots \ldots \ldots \ldots \ldots \ldots \ldots \ldots$

4.2 Cálculo de $c(f)$ e $d(f) \ldots \ldots \ldots \ldots \ldots$

4.3 Germes de corank 1. . . . . . . . . . . . . . . 47 


\section{Introdução}

Una questão relevante cm teoria de singularidades é o estudo dos invariantes associados a um germe de aplicação holomorfa. O primeiro invariante que surgiu foi o número de Milnor de um germe de uma função analítica $\int:\left(\mathbb{C}^{n}, 0\right) \rightarrow(\mathbb{C}, 0)$. Este invariante pode ser definido de várias maneiras. Algebricamonte é definido por

$$
\mu=\operatorname{dim}_{\mathbb{C}} \frac{\mathcal{O}_{n}}{\left\langle\frac{\partial f}{\partial z_{1}}, \frac{\partial f}{\partial z_{2}}, \ldots, \frac{\partial f}{\partial z_{n}}\right\rangle}
$$

Sua finitude é uma condição necessária e suficiente para a determinação finitá c, além disso, ele aparece associado à geometria da singularidade em duas formas: como números de pontos críticos de Morse em uma deformação estável e como o rank da homologia média da fibra de Milnor de $f$. Na teoria de singularidades de germes de aplicações $f:\left(\mathbb{C}^{n}, 0\right) \rightarrow\left(\mathbb{C}^{p}, 0\right)$ com $p>1$, não se encontra um invariante assim tão completo. Lma razão para isto está no fato que, enquanto para funções aparece apenas um tipo de singularidade estável, ou seja, a singularidade de Morse, para $p>1$ a complexidade da classificação dos germes e multigermes estáveis aumenta com $n$ e $p$.

As singularidades de germes de aplicaçõos do plano no plano foram primeiramente cstudadas por H. Whitney, em 1955 e, posteriormente, por J. Mather em 1970. Os trabalhos de Whitney e Mather versam sobre singularidades estáveis. A classificação dos germes simples de corank 1 (caso real ou complexo) foi obtida por J. Rieger em [21].

Quando um germe finitamente determinado não estável $f:\left(\mathbb{C}^{2}, 0\right) \rightarrow\left(\mathbb{C}^{2}, 0\right)$ é perturbado de modo a se tornar estável, um certo número de cúspides, $c(f)$, e dobras, $d(f)$, aparecem no discriminante, que ć uma curva. Os números $c(f)$ e $d(f)$ refletem a complexidade do germe inicial. Ainda, estes números são invariantes analíticos de $f$, isto é, se $f$ e $g$ são equivalentes por mudanças de coordenadas na fonte e na meta, então o número de cúspides (respectivamente de dobras) de $f$ e $g$ coincidem. Em [4], T. Gaffney e D. Mond apresentam fómulas para calcular estes invariantes. Mais ainda, eles mostram que 
oles são, de fato, invariantes topológicos, o que leva a condiçõos necessárias e suficientes para trivialidade topológica de farmílias de germes $\left(\mathbb{C}^{2}, 0\right) \rightarrow\left(\mathbb{C}^{2}, 0\right)$. Em $\left.\mid 21\right\}$, Rieger dá uma fórmula para estes invariantes em termos da multiplicidade local e dos invariantes $\mu$ e $\delta$ do conjunto dos pontos críticos. Finalmente, Gaffney e Mond, em [j], relacionam a $\mathcal{A}_{e}$-codimensão do germe $f$ com $c(f)$ e $d(f)$ e o número do Tjurina do discriminante.

O objetivo desta dissertação é apresentar as fórmulas que Gaffney e Mond obtiveram para $c(f)$ e $d(f)$. Para o desenvolvimento deste trabalho são necessários conhecimentos da teoria de singularidades e álgebra comutativa.

To capítulo 1 apresentamos alguns tópicos de teoria de singularidades tais como: germes de aplicaçôcs holomorfas, a álgebra $\mathcal{O}_{n}$, o módulo $\mathcal{O}_{n, p}$, A-equivalência, espaço tangente, determinação finita para germes, estabilidade de germes, equivalência de contato e desdobramentos.

O objetivo da capítulo 2 é apresentar os Anéis de Cohen-Macaulay. P’ara tanto, desenvolvemos alguns tópicos de Álgebra Comutativa básicos, definimos dimensão, grade e profundidade de um anel. Neste capítulo também abordamos o Teorema de Hilbert Burch.

No capitulo 3 estudamos germes de variedades analíticas complexas. Definimos o anel $\mathcal{O}_{X, 0}$, variedades de Cohen-Macaulay, normalização e intersecção completa com singularidade isolada. Além disso, abordamos os ideais de fitting e exibimos um algoritmo para calcular tais ideais quando $f$ é um germe de aplicação $\left(\mathbb{C}^{n}, 0\right) \rightarrow\left(\mathbb{C}^{n+1}, 0\right)$.

O capítulo 4 constitue a parte principal desta dissertação. Nele, definimos os invariantes $c(f)$ e $d(f)$, encontramos fórmulas para calculá-los e mostramos que $c(f)$ e $d(f)$ são invariantes topológicos. Além disso, obtemos relações entre estes invariantes e o grau do germe $f$. Finalmente, apresentamos fórmulas mais simples para o cálculo de $c(f)$ e $d(f)$ no caso particular em que o germe $f$ tom corank 1 . 


\section{Capítulo 1}

\section{Teoria de Singularidades}

Neste capítulo abordamos alguns tópicos da teoria de singularidades necessários para o desenvolvimento deste trabalho. Algumas demonstraçós são omitidas As principais reforencias são Cibson |10|, Golubitsky |11|, Martinet |18| e Wall |29|.

\subsection{Germes e $\mathcal{A}$-equivalência}

Scja $S=\left\{x_{1}, \ldots x_{k}\right\} \subset \mathbb{C}^{n}$. Consideremos o conjunto das aplicaŗõos holomorfas definidas numa vizinhança aberta de $S$ em $\mathbb{C}^{n}$ com valores em $\mathbb{C}^{p}$. Introduzimos neste conjunto a seguinte relação de cquivalôncia: dadas $f: U \rightarrow \mathbb{C}^{p}$ e $g: V \rightarrow \mathbb{C}^{p}$ en tal conjunto, dizemos que $f$ e $g$ são equivalentes se existe uma vizinhança aberta $W \subset U \cap V$ de $S$ tal que $\left.f\right|_{w}=\left.g\right|_{w}$.

Definiçāo 1.1.1 O multigerme de uma aplicaçăo holomorfa $f: U \rightarrow \mathbb{C}^{p}$, onde $U$ é uma vizinhança aberta de $S \subset \mathbb{C}^{n}$, ć a classe de equivalência de f́ segundo a relação de equivalência definida acima. Denotumos o multigerme de $f$ por $f:\left(\mathbb{C}^{n}, S\right) \rightarrow\left(\mathbb{N}^{p}, y\right)$. Se $S=\{x\}$, esta classe de equivalencia é chamada de germe, $x$ é chamado de jonle do germe e $y=f(x)$ é chamado de meta do germe.

Dados dois germes $f:\left(\mathbb{C}^{n}, x\right) \rightarrow\left(\mathbb{C}^{p}, y\right)$ e $g:\left(\mathbb{C}^{p}, y\right) \rightarrow\left(\mathbb{C}^{y}, z\right)$ podemos obter o germe $f \circ g:\left(\mathbb{C}^{n}, x\right) \rightarrow\left(\mathbb{C}^{q}, z\right)$ tomando representantes convenientes de $f$ e $g$, diganos $\hat{f}$ e $g$; de forma que $\widehat{g} \circ \widehat{f}$ esteja bem definida. O germe $g \circ f$ é germe de $\widehat{g} \circ \widehat{f}$.

A derivada do germe $f:\left(\mathbb{C}^{n}, x\right) \rightarrow\left(\mathbb{C}^{p}, y\right)$ em $x$ é a derivada em $x$ de qualquer representante de $f$. Notemos que esta definiçăo independe da escolha do representante.

Dizemos que $f:\left(\mathbb{C}^{n}, x\right) \rightarrow\left(\mathbb{C}^{p}, y\right)$ o um germe de difeomorfismo se um de seus representantes, e portanto todos, é um difeomorfismo local em $x$. 
Teorema 1.1.2 (Teorema da Função Inversa para Germes) Consideremos o germe. $f:\left(\mathbb{C}^{n}, x\right) \rightarrow\left(\mathbb{C}^{p}, y\right)$. O germe $f$ ó de difeomorfismo se, e somente se, sua derivada $d_{x} f: \mathbb{C}^{n} \rightarrow \mathbb{C}^{p}$ a um isomorfismo.

Definiçāo 1.1.3 O rank de um germe $f:\left(\mathbb{C}^{n}, x\right) \rightarrow\left(\mathbb{C}^{p}, y\right)$ o o runk da matriz de $d_{x} f$ e E denotado por rank f. O corank de $f$. o qual denotamos por corank fo c definido como sendo $\min \{n, p\}-\operatorname{rank} f$.

Consideremos o germe $f:\left(\mathbb{C}^{n}, x\right) \rightarrow\left(\mathbb{C}^{p}, y\right)$. Dizemos que $f$ é germe de imersão se rank $f=n$ e de submersão se rank $f=p$. O germe $f$ c dito scr singular ou singularidade se nào é submersão e nem imersão, on seja, rank $f<\min \{n, p\}$. Portanto, $f$ é um germe de difeomorfismo se é germe de imersão e de submersão.

Definição 1.1.4 Dois germes $f_{1}:\left(\mathbb{C}^{n}, x_{1}\right) \rightarrow\left(\mathbb{C}^{p} \cdot y_{1}\right) e f_{2}:\left(\mathbb{C}^{n}, x_{2}\right) \rightarrow\left(\mathbb{C}^{p}, y_{2}\right)$ sũo ditos serem A-equivalentes, e denolamos por $f_{1} \sim_{\mathcal{A}} f_{2}$, se existem germes de difcomorfismos $\phi:\left(\mathbb{C}^{n}, x_{1}\right) \rightarrow\left(\mathbb{C}^{n}, x_{2}\right)$ e $:\left(\mathbb{C}^{p}, y_{1}\right) \rightarrow\left(\mathbb{C}^{p}, y_{2}\right)$ para os quais o diagrama

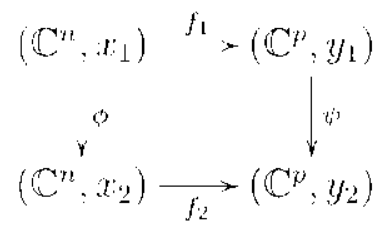

é comutativo, ou seja, $f_{2}=\dot{\psi} \circ f_{1} \circ \phi^{-1}$

Como consequência da Definiçào 1.1.4. temos que todo germe $\left(\mathbb{C}^{n}, x\right) \rightarrow\left(\mathbb{C}^{p}, y\right)$ é $\mathcal{A}$-equivalente a algum germe $\left(\mathbb{C}^{n}, 0\right) \rightarrow\left(\mathbb{C}^{\mu}, 0\right)$.

O conjunto dos germes $\left(\mathbb{C}^{n}, 0\right) \rightarrow\left(\mathbb{C}^{p}, y\right)$ e denotado por $\mathcal{O}_{n, y}$. Analogamente, podemus definir o conjunto dos germes $f:\left(\mathbb{R}^{n}, 0\right) \rightarrow\left(\mathbb{R}^{p}, y\right)$, que denotamos por $\mathcal{E}_{n, 7,}$. Quando $p=1$, denotamos por $\mathcal{O}_{n}$ e $\mathcal{E}_{n}$ respectivamente. O conjunto dos germes de difeomorlismos de $\mathcal{O}_{n, n}$ é um grupo com a operação de composição e é denotado por Diff( $\mathbb{C}^{n}$ ) on $D^{n}$.

Iemos que $\mathcal{O}_{n}$ é un espaço vetorial complexo e possui estrutura de anel local. O ideal maximal de $\mathcal{O}_{n}$ é $\mathcal{M}_{n}=\left\{g \in \mathcal{O}_{n} / g(0)=0\right\}$. Portanto, $\mathcal{O}_{n}$ é uma $\mathbb{C}$-álgebra.

Analogamente, $\mathcal{E}_{n}$ ć um espaço vetorial real cum estrutura de and local e seu ideal maximal é $\mathcal{M}_{n}{ }^{\mathbb{R}} \ldots\left\{g \in \mathcal{E}_{n} / g(0)=0\right\}$. Assim, $\mathcal{E}_{n}$ é uma $\mathbb{R}$-álgebra.

Notenos que $\mathcal{O}_{n, p}$ ém $\mathcal{O}_{n}$-módulo live. De fato, se $f \in \mathcal{O}_{n, p}$ então $f \quad\left(f_{1}, f_{2}, \ldots, f_{p}\right)$, onde $f_{i} \in \mathcal{O}_{n}$ para $i=1,2 \ldots, p$. Logo $\mathcal{O}_{n, p} \simeq \mathcal{O}_{n} \circlearrowleft \mathcal{O}_{n} \odot \cdots \oplus \mathcal{O}_{n}$. Analogamente, $\mathcal{E}_{n, p}$ o um $\mathcal{E}_{n}$-módulo livre.

Denotamos por $J^{k}(n, p)$ o espacso vetorial complexo das aplicacoes $f: \mathbb{C}^{n}, \mathbb{C}^{p}$ onde cada uma das componentes $f_{1}, f_{2}, \ldots, f_{p}$ é um polinômio de grau menor ou igual a ki nas 
coordenadas $x_{1}, x_{2}, \ldots, x_{n}$ com termo constante nulo. Os elementos do $J^{k}(n, p)$ são ditos k-jatos.

Sejam $f: U \subset \mathbb{C}^{n} \rightarrow \mathbb{C}^{n}$ uma aplicaçio holomorfa, onde $U$ é um aberto de $\mathbb{C}^{n}$ e $a \in U$. $\Lambda$ expansão em série de potèncias de $\int(x-a)$ em torno de o c dada por

$$
f(a)+d_{u} f x-\frac{1}{2 !} d_{u}^{2} f x^{2}+\cdots
$$

Definição 1.1.5 Sejam $f \in \mathcal{O}_{n, p} \in h \in \mathbb{Z}, k \geq 1$. O k-jato de $f$ em a, denotado por $j^{k} f(a)$, é definido por

$$
d_{n} f \cdot x+\frac{1}{2 !} d_{a}^{2} f^{\prime} \cdot x^{2}+\cdots+\frac{1}{k !} d d_{n}^{k} f \cdot x^{k}
$$

isto $\hat{e}, j^{k} f(a)$ é a expansăo em série de polências de $f(x+a)-f(a)$ cm torno de 0 truncada no termo de grau $k$, onde f é umr representante do germe.

\subsection{Espaço Tangente}

Definição 1.2.1 Sejam G um grupo : $M$ um conjunto. Uma ação do grupo $G$ no conjunto $M$ é uma aplicasúo $\varphi: G \times M \rightarrow M$ (escrevemos $\varphi(g, x)=$ g.x) salisfazendo

1. $1 . x \therefore x \forall x \in M$, onde 1 ó o elemento neutro de $G$.

g. $(g h) . x=g \cdot(h, x) \forall g, h \in G, x \in M$.

Una ação incluz una relação de equivaloncia em $M$ da seguinte forma: dados $x, y \in M$, dizemos que $x$ é equivalente a $y$ se existe $y \in G$ tal que $y-g . x$. A classe de equivalôncia de $x \in M$ segundo esta relação de equivalência é chamada de órbita de $x$ e é denotada por $G . x=\{g x / g \in G\}$.

A $\mathcal{A}$-equivalência cm $\mathcal{O}_{n, p}$ provém de uma ação. De lato, consideremos o grupo $\mathcal{A}=$ $\operatorname{Diff}\left(\mathbb{C}^{n}\right) \times \operatorname{Diff}\left(\mathbb{C}^{p}\right)$. Este grupo age em $\mathcal{O}_{n, p}$ da seguinte forma

$$
\begin{aligned}
\mathcal{A} \times \mathcal{O}_{n, p} & \rightarrow \mathcal{O}_{n, p} \\
((\phi, i) . f) & \longmapsto \psi \circ f \circ \phi^{-1}
\end{aligned}
$$

Logo, dois germes $f, g \in \mathcal{O}_{n, p}$ são A-equivalentes se, e somente se, $f$ e $g$ estão na mesma órbita segundo a ação acima.

Definição 1.2.2 Um grupo de Lì G á um grupo que é uma variedade diferenciável e a.s aplicaseres

$$
\begin{array}{rlrl}
G \times G & \longrightarrow G & G & \longrightarrow G \\
(x, y) & \longmapsto x y & x & \longmapsto x^{-1}
\end{array}
$$


säo de classe Co.

Definiçāo 1.2.3 Uma açăo de um yrupo de Lie (' numa martedade diferenciánel Mé uma açao $\varphi: G \times M \cdots+M$ que é de classe $C^{-x}$.

No que segue vamos definir uma relaçào de equivalência entre k-jatos de maneira análoga à definida para gemmes.

Definição 1.2.4 Dois $k$-jatos $f, g \in J^{k}(n, p)$ săo ditos serem equivalentes se existem $\phi \in$ $\operatorname{Diff}\left(\mathbb{C}^{n}\right)$ e $\dot{\psi} \in \operatorname{Diff}\left(\mathbb{C}^{\prime \prime}\right)$ tais que $j^{k}\left(z^{\circ} \circ f \circ g^{-1}\right)(0)=j^{k} g(0)$.

A relaça de equivalência definida acima provém de uma ação de um grupo de Lie na variedade $J^{k}(n, p)$. De lato, consideremos o conjunto dos $k$-jatos de elementos de Diff( $\left.\mathbb{C}^{n}\right)$. Este conjunto é um grupo com a seguinte operaçä:

$$
j^{k} h_{1}(0) * j^{k} h_{2}(0)-j^{k}\left(h_{1} \circ h_{2}\right)(0)
$$

Denotames aste grupo por $D_{n}^{k}$. Este grupo é um conjunto aberto no espaço vetorial $J^{k}(n, n)$. Portanto, co una variedade diferenciável e o seu espaço tangente em qualquer ponto é $J^{k}(n, n)$. Logo, $D_{n}^{k}$ é um grupo de Lie. Segue que $\mathcal{A}^{k}=D_{n}^{k} \times D_{p}^{k}$, que é o grrupo dos $k$-jatos de elementos de $\mathcal{A}$, é um grupo de Lie, pois o produto cartesiano de grupos de Lie é um grupo de Lie. O grupo $\mathcal{A}^{k}$ age em $J^{k}(n, p)$ da forma:

$$
\begin{aligned}
\varphi: \mathcal{A}^{k} \times J^{k}(n, p) & \rightarrow J^{k}(n, p) \\
\left(j^{k} \phi(0), j^{k}((0), f)\right. & \longmapsto j^{k}\left(\varphi^{\prime} \circ f \circ \phi^{-1}\right)(0)
\end{aligned}
$$

Fin geral as orbitas de um grupo de Lie muma variedade nato sào subvariodades. No entanto, se supusermos que as órbitas săo subvariedades, o resultado seguinte descreve o espaço tangente a uma órbita.

Proposição 1.2.5 Seja $\varphi: G \times M \rightarrow M$ uma ação de um grupo de Lie $G$ numa variedade M. Suponhamos que as órbitas em M segundo esta ação sejam subvariedades de

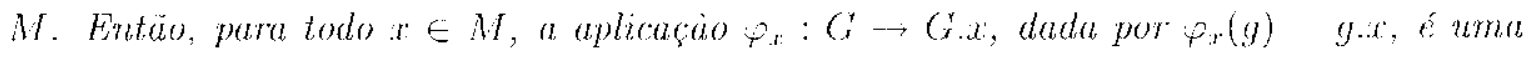
subnersĕo.

Demonstração Dado $x \in M$, mostremos primeiramente que a aplicação $\varphi_{x}$ tem o mesmo rank cm todo elemento de $G$. Para isto, basta mostrar que o rank de $\varphi_{x}$ em qualquer $h \in G$ coincide com o rank de px no elemento nentro 1 de $G$. Seja $\theta: G \rightarrow G$ o difeomorfismo dado por $\theta(g)-h g$ e $: M, M O$ dilcomorfismo dado por $\operatorname{lo}(y)=h . y$. 
Consideremos o diagrama

$$
\begin{aligned}
& G^{p}>G x
\end{aligned}
$$

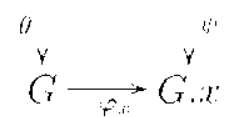

Dà definição de ação, ste diagrama é comutativo. Aplicando a regra da cadeia obtemos o diagrama comutativo

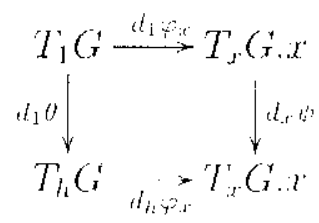

Como 0 o te sâo difeomorfismos, $d_{1} \theta$ o $d_{x}$ s saio isomorfismos e, portanto,

$$
\operatorname{dim} d_{1} \varphi_{x}\left(T_{1}(r)=\operatorname{dim} d_{h} \varphi_{x}\left(T_{h} G\right)\right.
$$

Logo o rank de $\varphi_{x}$ em ho igual ao rank de $\varphi_{x}$ em 1. Basta mostrar agora que $\varphi_{x}$ ó submersão em algum ponto de $G$, mas isto segue do Teorema de Sard (ver [10]).

A seguir vanos obter o espaço tangente à orbita de $f \in J^{k}(n, p)$ segundo a ação do grupo $\mathcal{A}^{k}$. Denotamos tal espaço por $T \mathcal{A}^{k} . f$. Pela proposiçào acima, temos que $T \mathcal{A}^{k} f$ é a inagen da derivada da aplicação $\varphi_{j}$ em $I=\left(I_{n}, I_{p}\right)$, onde $I_{n}$ e $I_{p}$ são as identidades em $\mathbb{C}^{n}$ a $\mathbb{C}^{\prime \prime}$ respectivamente:

$$
\begin{gathered}
T \mathcal{A}^{k} \cdot f=d_{l \varphi_{f}}\left(T_{l}\left(D_{n}^{k} \times D_{p}^{k}\right)\right)=d_{l \varphi f}\left(T_{I_{n}} D_{n}^{k} \times T_{l_{i}} D_{p}^{k}\right) \\
=d_{I \varphi_{f}}\left(T_{l_{n}} D_{n}^{k} \times\{0\}+\{0\} \times T_{l^{\prime}} D_{p}^{k}\right)=d_{I \psi f}\left(J^{k}(n, n) \times\{0\}\right)-d_{l} \varphi_{f}\left(\{0\} \times J^{k}(p, p)\right)
\end{gathered}
$$

Consideremos

$$
\begin{aligned}
& \xi_{1}: D_{n}^{k} \longrightarrow J^{k}(n, p) \\
& j^{k} \phi(0) \longrightarrow j^{k}\left(f \circ \phi^{-1}\right)(0) \\
& \xi_{2}: D_{p}^{k} \longrightarrow j^{k}(n, p) \\
& j^{k} \gamma_{1}(0) \longrightarrow j^{k}(\varphi \circ f)(0)
\end{aligned}
$$

Notemos que $\xi_{1}$ é a composição da aplicação inversão em $D_{n}^{k}$ com a aplicação

$$
\begin{aligned}
\zeta: \Gamma_{n}^{k} & \longrightarrow J^{k}(n \cdot p) \\
j^{k} \phi(0) & \longmapsto j^{k}(f \circ \emptyset)(0)
\end{aligned}
$$


Logo a imagem de $d_{I_{n}} \xi_{1}$ coincide com a imagen cle $d_{I_{n}} \zeta$. Temos que

$$
d_{I \varphi f}\left(J^{k}(n, n) \times\{0\}\right)=d_{l_{n}} \xi_{1}\left(J^{k}(n, n)\right)=d_{I_{n}} \zeta\left(J^{k}(n, n)\right)
$$

De fato, se $u \in J^{k}(n, n)=T_{l_{n}} D_{n}^{k}$, cntäo existe una curva $\lambda:(-\epsilon, \epsilon) \rightarrow D_{n}^{k}$ tal que $\lambda(0)=I_{n}$ e $\lambda^{\prime}(0) \cdots$ u. Seja $\Lambda:(\cdots \epsilon, \epsilon) \rightarrow D_{n}^{k} \times D_{p}^{k}$ dada por $\Lambda(t)=\left(\lambda(l), I_{p}\right)$. Notemos (que $\Lambda(0)-\left(I_{n}, I_{p}\right)=I$ \& $\Lambda^{\prime}(0)=(u, 0)$. Portanto

$$
d_{1} \varphi_{f}(u, 0)=\frac{d(\varphi, \circ \Lambda)}{d l}(0) \ldots \frac{d\left(\xi_{1} \circ \lambda\right)}{d t}(0)=d_{l_{n}} \xi_{1}(u)
$$

Basta cntão calcular $d_{I_{n}} \zeta\left(J^{k}(n, n)\right)$. Seja $g-:\left(g_{1}, \ldots, g_{n}\right) \in J^{k}(n, n)$. Para um valor de $t$ suficientemente pecfueno temos que $I_{n}-$ tgé invertível. assim, para $\varepsilon>0$ suficientemente pecueno temos a curva $\alpha:(-t, \epsilon) \rightarrow D_{n}^{k}$ dada por a $(l)=I_{n}+t g$. Ainda

$$
\zeta \circ \alpha(t)=\zeta\left(I_{n}+l_{g}\right)=j^{h}\left(f \circ\left(I_{n}+l g\right)\right)(0)=j^{k} f\left(x_{1}+t g_{1}, \ldots, x_{n}+\operatorname{tg}\right)(0)
$$

Logo

$$
\begin{gathered}
\frac{d(\zeta \circ(t)}{d t}(0)=\lim _{t \cdot 0} \frac{(\zeta \circ(x)(t)-(\zeta \circ(x)(0)}{t} \\
=\lim _{t \rightarrow 0} \frac{j^{k} f\left(x_{1}-t g_{1}, \ldots x_{n}-l g_{n}\right)(0)-j^{k} f\left(x_{1}, \ldots, x_{n}\right)(0)}{l} \\
=j^{k}\left(\lim _{t \rightarrow 0} \frac{f\left(x_{1}-l g_{1} \ldots \ldots x_{n}+l g_{n}\right)-f\left(x_{1}, \ldots, x_{n}\right)}{t}\right)(0) \\
=j^{k}\left(\left.\frac{\partial f}{\partial t}\left(x_{1}+l g_{1}, \ldots, x_{n}+t g_{n}\right)\right|_{\ell=0}\right)(0) \\
j^{k}\left(\left.\sum_{i=1}^{n} \frac{\partial f}{\partial x_{i}}(x+l g) g_{i}\right|_{t-0}\right)(0)=j^{k}\left(\sum_{i=1}^{n} \frac{\partial f}{\partial x_{i}} g_{i}\right)(0)
\end{gathered}
$$

Analogamente,

$$
d_{l \varphi f}\left(\{0\} \times J^{k}(p, p)\right)=d_{I_{p}} \xi_{2}\left(J^{k}(p, p)\right)
$$

Calculemos então $d_{l_{p}} \varepsilon_{2}\left(J^{k}(p, p)\right)$. Tomando $h \in J^{k}(p, p)$, podemos considerar, assim

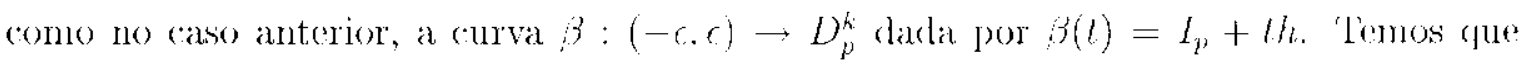
$\xi_{2} \circ \beta(t)=\xi_{2}\left(I_{p}+t h\right)=j^{k}\left(\left(I_{p}+t h\right) \circ f\right)(0)=j^{k}(f+t h \circ f)(0)$.

$$
\begin{aligned}
& d_{h_{1}} \xi_{2}(h)=\frac{d\left(\xi_{2} \circ \beta\right)}{d t}(0)=\lim _{t \rightarrow 0} \frac{\left(\xi_{2} \circ \beta\right)(t)-\left(\xi_{2} \circ \beta\right)(0)}{t} \\
& =\lim _{t \rightarrow 0} \frac{j^{k}(f-t h \circ f)(0)-j^{k}(f)(0)}{t}=j^{k}\left(\lim _{t \cdot 0} \frac{f+t h \circ f \cdots f}{t}\right)(0)=j^{k}(h \circ f)(0)
\end{aligned}
$$


Logo $d_{l_{p}} \xi_{2}\left(J^{k}(p, p)\right)=\left\{j^{k}(h \circ f)(0) / h \in J^{k}(p, p)\right\}$. Segue que o espaço tangente à órbita de $f$ cm $f^{k}(n, p)$ segundo a açäo do grrupo $\mathcal{A}^{k}$ a

$$
T \mathcal{A}^{k} \cdot f=\left\{j^{k}\left(\sum_{i=1}^{n} \frac{\partial f}{\partial x_{i}} g_{h}\right)(0) / g \quad\left(g_{1}, \ldots, g_{n}\right) \in J^{k}(n, n)\right\} \quad\left\{j^{k}(h \circ f)(0) / h \in f^{k}(p, p)\right\}
$$

Consideremos a órbila de um germe segundo a ação do grupo $\mathcal{A}$. Lste grupo não c de Lir e as órbitats nào são subvarieclades. Motivados pelo caso dos jatos definimos:

Definição 1.2.6 Seju $f \in \mathcal{O}_{n, p}$.

1. O espaço tangente à ónbita de f segundo a açäo do grupo $\mathcal{A}$ é

$$
\text { T'A.f }=\left\{\sum_{i=1}^{n} \frac{\partial f}{\partial x_{i}} g_{i} / g=\left(g_{1} \ldots, g_{n}\right) \in \mathcal{M}_{n} \mathcal{O}_{n, n}\right\}+\left\{h \circ f / h \in \mathcal{M}_{p} \mathcal{O}_{p, n}\right\}
$$

2. O espaço langente restendido ó

$$
T \mathcal{A}_{t} . f=\left\{\sum_{i-1}^{n} \frac{\partial f}{\partial x_{i}} g_{i} / g=\left(g_{1} \ldots, g_{n}\right) \in \mathcal{O}_{n, n}\right\}+\left\{h \circ f / h \in \mathcal{O}_{p, p}\right\}
$$

Detinimos a $\mathcal{A}$-codimensão de $f$, ou codimensão de $f$, como sendo a dimensäo de

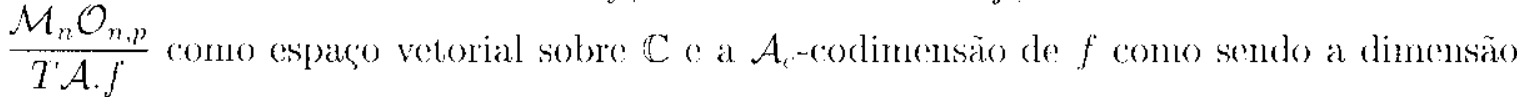
de $\frac{\mathcal{O}_{n, p}}{T \mathcal{A}_{e} \cdot f}$ como espagco vetorial solore $\mathbb{C}$.

Descrevemos a seguir os espaços tangentes da Definição acima usando a linguagem de campos de vetores.

Definição 1.2.7 Seja $M$ uma variedade diferenciável. O fibrado tangente a $M$, denotado por $T M$, é o conjunto $\left\{(x, v) / v \in T_{x} M\right\}$.

Definição 1.2.8 Sejam $U$ um subconjunto aberto de $\mathbb{C}^{n}$ e $f: U \cdots, \mathbb{C}^{p}$ uma aplicaçăo holomorfa. A aplicaçäo tangente, denotada por $T_{f}$, é definida por

$$
\begin{array}{rll}
T_{f}: T \mathbb{C}^{n} & \quad ; & T \mathbb{C}^{n} \\
(x, v) & \cdots \rightarrow & \left(f(x), l_{r} f(v)\right)
\end{array}
$$

Denotamos por $\pi_{n}$ a projeção do fibrado tangente a $\mathbb{C}^{n}$ sobre $\mathbb{C}^{n}$

$$
\begin{array}{r}
\pi_{n}: T \mathbb{C}^{n} \longrightarrow \mathbb{C}^{n} \\
(x, v), \quad x
\end{array}
$$


Definição 1.2.9 Sejam $U$ um subconjunto aberto de $\mathbb{C}^{n}$ e $f: U \rightarrow \mathbb{C}^{p}$ uma aplicaçäo holomorfa. Um campo de vetores ao longo de $f$ é uma aplicaçäo holomorfa $w: U$.., $T \mathbb{C}^{p}$ que satisfaz $\pi_{p} \circ \omega=f$, ou seja, para cada $x \in U, \omega(x)=(f(x), v)$, onde $x$ é um vetor de. $T_{f(x)} \mathbb{C}^{p}$

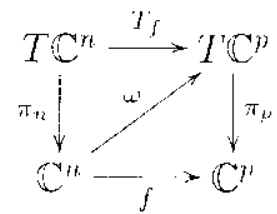

Dada $f \in \mathcal{O}_{n, p}$, denotamos por $\theta(f)$ o conjunto dos germes em 0 dos campos de vetores au longo de $f$.

So $I_{n}$ a a identidade em $\mathbb{C}^{n}$, denotamos por $\theta(n)$ o conjunto $\theta\left(I_{n}\right)$. Notemes que existe uma identificaçăo natural de $T^{\mathbb{C}}$ com $\mathbb{C}^{n} \times \mathbb{C}^{n}$ e de $\theta(f)$ com $\mathcal{O}_{n, p}$.

Os segnintes diagramas são comutativus:

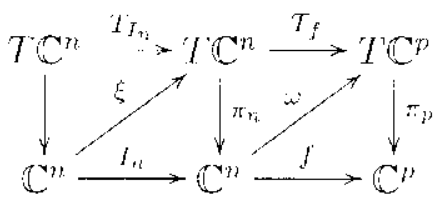

Se $g(x)=(x, \bar{\xi}(x))$ então

$$
T f \circ \xi(x)=T f(x, \bar{\xi}(x))=\left(f(x), d_{x} f(\bar{\xi}(x))\right)
$$

Onde o germe $\xi$ é um elemento de $\theta(n)$ o o germe de $T f \circ \xi$ é um elemento de $\theta(f)$. Definimos então

$$
\begin{aligned}
t f: \theta(n) & \longrightarrow \theta(f) \\
\xi & , T f \circ \xi
\end{aligned}
$$

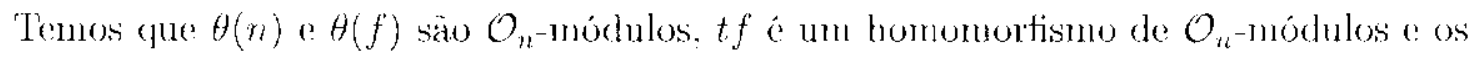
seguintes subeonjuntos de $\theta(f)$ säo submódulos.

$$
\begin{gathered}
\left\{\sum_{i=1}^{n} \frac{\partial j}{\partial x_{i}} g_{i} / g=\left(g_{1}, \ldots, g_{n}\right) \in \mathcal{M}_{n} \mathcal{O}_{n, n}\right\}=\left\{d_{x} f(g) / g \in \mathcal{M}_{n} \theta(n)\right\}=t f\left(\mathcal{M}_{n} \theta(n)\right) \\
\left\{\sum_{i=1}^{n} \frac{\partial f}{\partial x_{i}} g_{i} / g=\left(g_{1}, \ldots, g_{n}\right) \in \mathcal{O}_{n, n}\right\}=\left\{d_{x} f(g) / g \in \theta(n)\right\}=\operatorname{tf}(\theta(n))
\end{gathered}
$$

Cm germe $f \in \mathcal{O}_{n, j}$ induz o homomorfismo de álgobras

$$
\begin{aligned}
& f^{*}: \mathcal{O}_{p}, \quad, \mathcal{O}_{w} \\
& \text { h. } 1 \text { hof }
\end{aligned}
$$


Dado $M$ um $\mathcal{O}_{n}$-módulo, podemos definir em $M$ uma estrutura de $\mathcal{O}_{p}$-módulo via $f^{\star}$. Assim, $\theta(f), t f\left(\mathcal{M}_{n} \theta(n)\right)$ e $\ell f(\theta(n))$ possuem estrutura de $\mathcal{O}_{p}$-nódulo via $f^{*}$. Definimos o homomortismo de $\mathcal{O}_{p}$-módulos (via $f^{*}$ )

$$
\begin{aligned}
\omega f: \theta(p) & \longrightarrow \theta(f) \\
\eta & \longmapsto \eta \circ f
\end{aligned}
$$

Assim,

$$
\begin{gathered}
\left\{h \circ f / h \in \mathcal{M}_{p}, \mathcal{O}_{p, p}\right\}=\left\{\omega f(h) / h \in \mathcal{M}_{p} \theta(p)\right\}=\omega f\left(\mathcal{M}_{p} \theta(p)\right) \\
\left\{h \circ f / h \in \mathcal{O}_{p, p}\right\}-\{\omega f(h) / h \in O(p)\} \cdots \omega f(0(p))
\end{gathered}
$$

Os subconjuntos acina são $\mathcal{O}_{p}$-submódulos de $\theta\left(\int\right)$. Além disso,

$$
\begin{gathered}
\text { TA.f. } t f\left(\mathcal{M}_{n} O(n)\right)+\omega f\left(\mathcal{M}_{p} 0(p)\right) \\
T \mathcal{A}_{c} f=t f(\theta(n))+\omega f(\theta(p))
\end{gathered}
$$

Notemos que T A.f não é um $\mathcal{O}_{n}$-submódulo mas um $\mathcal{O}_{p}$-submódulo via $f^{*}$.

Definição 1.2.10 Seja $f \in \mathcal{O}_{n . p .}$. Dizemos que $f$ é estável se para cadaw $\in O(f)$ eristem $\xi \in \theta(n)$ e $\eta \in \theta(p)$ tais que

$$
w-l f(\xi)+w f(\eta)
$$

ou seju, $f$ é estável se $T \mathcal{A}_{c} . f=\theta(f)$.

\subsection{Determinação Finita e Desdobramentos}

Definição 1.3.1 Dizemos que um germe $f \in \mathcal{O}_{n, p}$ é $k$-determinado, para algum $k \in \mathbb{N}$, se para lodo $g \in \mathcal{O}_{n, p}$ lal que $j^{k} g(0)=j^{k} f(0)$ lemos que g é $\mathcal{A}$-equivalente a $f$. Dizemos que $f$ é finitamente determinado se $f$ é $k$-determinado para algum $k \in \mathbb{N}$.

O teorema seguinte é un resultado de Mather encontrade em [29].

Teorema 1.3.2 Seja $f \in \mathcal{O}_{n, p}$. Fntão säo equivalentes

i) Ogerme fé finitamente determinado,

ii) Existe um inteiro positivo $k$ tal que $\mathcal{M}_{n}^{k} \subset$ I'A.f,

iii) $A \mathcal{A}$-codimensaio de $f$ finitr.

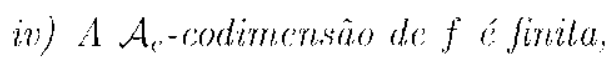


O resultado seguinte é uma caracterização geométrica dos gerrres finitamente deterrminados.

Teorema 1.3.3 Um germé $f \in \mathcal{M}_{n} \mathcal{O}_{n, p}$ é fintamente determinado se, e somente se, exisle uma vizinhança $V$ de $0 \mathrm{em} \mathbb{C}^{n}$ tal que para lodo subconjunto finilo $S \subset V-\{0\}$, 0 multigerme de j em S éstavel.

Seja $f_{0} \in \mathcal{M}_{n} \mathcal{O}_{n, p}$. Un desdobramento a s-parâmetros de $f_{0}$ o $u m$ germe de uma aplicaça holomorfa

$$
\begin{aligned}
F:\left(\mathbb{C}^{s} \times \mathbb{C}^{n}: 0\right) & \longrightarrow\left(\mathbb{C}^{s} \times \mathbb{C}^{p}, 0\right) \\
(u, x) & \longrightarrow(u, f(u, x))
\end{aligned}
$$

satisfazendo $f(0, x)=f_{0}(x)$. O germe $f:\left(\mathbb{C}^{*} \times \mathbb{C}^{n}, 0\right) \rightarrow\left(\mathbb{C}^{*}, 0\right)$ a chamado de deformaçio de $\int_{0}$.

Dois desdobramentos $F, G:\left(\mathbb{C}^{*} \times \mathbb{C}^{n}, 0\right) \rightarrow\left(\mathbb{C}^{s} \times \mathbb{C}^{p}, 0\right)$ de $f_{10}$ são isomorfos se existem germes de difcomorfismos $0:\left(\mathbb{C}^{s} \times \mathbb{C}^{n}, 0\right) \rightarrow\left(\mathbb{C}^{s} \times \mathbb{C}^{n}, 0\right)$ e $t:\left(\mathbb{C}^{s} \times \mathbb{C}^{p}, 0\right) \rightarrow\left(\mathbb{C}^{s} \times \mathbb{C}^{p}, 0\right)$, desdobramentos a s-paranmetros dos germes das identidades em $\mathbb{C}^{n}$ e $\mathbb{C}^{p}$ respectivamente, tais que $G=\dot{\psi} \circ \mathrm{F} \circ \phi^{-1}$.

Sejarm $F$ um desdobramento a s-parámetros de $f_{0} \in \mathcal{M}_{n} \mathcal{O}_{n, p}$ e $h:\left(\mathbb{C}^{t}, 0\right) \rightarrow\left(\mathbb{C}^{s}: 0\right) \mathrm{um}$ germe. O pull-back de $F$ por $h$, denotado por $h^{*} F$, é definido como sendo o desdobramento (le fo a t-parámetros dado por

$$
\begin{aligned}
h^{*} F:\left(\mathbb{C}^{l} \times \mathbb{C}^{n}, 0\right) & \longrightarrow\left(\mathbb{C}^{l} \times \mathbb{C}^{p}, 0\right) \\
(u, x) & \longrightarrow(v, f(h, v), x))
\end{aligned}
$$

Se $G$ é desdobramento a t-paránetros de $f_{0}$, dizemos que $G$ e induzido de $F$ se existe um germe $h:\left(\mathbb{C}^{\prime}, 0\right) \rightarrow\left(\mathbb{C}^{s}, 0\right)$ tal que $G$ c isomorfo a $h^{*} F$. En particular, se $h:\left(\mathbb{C}^{s}, 0\right) \rightarrow$ $\left(\mathbb{C}^{s}, 0\right)$ é um germe de difeomorfimmo, dizenos que $F^{\prime}$ e $G$ são equivalentes.

Dizemos que $F$ e um desdobramento versal de $f_{0}$ se todos os desdobramentos de $f_{0}$ são induzidos de $F$. Quando $F$ é versal com um número mínino de paràmetros dizemos (que $F$ é miniversal.

$O$ desdobramento $F$ e dito ser trivial so o isomorfo ao desdobramento constante $G$ : $\left(\mathbb{C}^{4} \times \mathbb{C}^{n}, 0\right) \rightarrow\left(\mathbb{C}^{*} \times \mathbb{C}^{p}, 0\right), C(u, x)=\left(u, f_{0}(x)\right)$.

Definição 1.3.4 Seja $f_{0} \in \mathcal{M}_{n} \mathcal{O}_{n . p}$. Um desdobramenlo a s-parâmetros de $f_{0}$ $F^{\prime}:\left(\mathbb{C}^{s} \times \mathbb{C}^{n}, 0\right) \rightarrow\left(\mathbb{C}^{s} \times \mathbb{C}^{p}, 0\right)$ e topologicamente trivial se existem $\omega:\left(\mathbb{C}^{s} \times \mathbb{C}^{n}, 0\right) \rightarrow$ $\left(\mathbb{C}^{*} \times \mathbb{C}^{n}, 0\right)$ e bramentos da identidade em $\mathbb{C}^{n}$ e $\mathbb{C}^{p}$ respectivamente, tais que $G=\xi \circ F \circ \sigma^{-1}$, onde $G$ é o desdobramento constante. 
Scja $F:\left(\mathbb{C}^{s} \times \mathbb{C}^{n}, 0\right) \rightarrow\left(\mathbb{C}^{s} \times \mathbb{C}^{p}, 0\right), F(u, x)=(u, f(u, x))$, um desdobramento topologicamente trivial de $f_{0} \in \mathcal{M}_{n} \mathcal{O}_{u, p}$ a s-parametros. Então as aplicaçoes $f_{u}$ : $\left(\mathbb{C}^{\prime \prime}, 0\right) \rightarrow\left(\mathbb{C}^{p}:(0)\right.$. diadas por $f_{i t}(x)=f(u, x)$ sĩo topologiamente equivalentes. De fato, se $\phi(x, y)=(u, \widetilde{\phi}(u, x))$ o $(u, y)=(u, \tilde{v}(u, y))$ são como acima, temos

$$
f_{0} \circ y_{u} \circ f_{u}
$$

onde $\tilde{\phi}(x)=\tilde{\phi}(u, x)$ e $\tilde{u}(y)=\tilde{\phi}(u, y)$.

\subsection{Teorema da Preparação}

Lema 1.4.1 (Lema de Hadamard) Sejam U uma vizinhança conexa de o em $\mathbb{C}^{n} \in$ $f: U \times \mathbb{C}^{q}, \mathbb{C}$ uma funçüo analitica tal que $f(0, y) \cdots$ o para lodo $y \in \mathbb{C}^{q}$. Entăo $f\left(x_{1}, \ldots, x_{n}, y_{1}, \ldots, y_{y}\right)=x_{1} f_{1}(x, y)+\cdots+x_{n} f_{n}(x, y)$ onde $f_{1}, \ldots, f_{n}$ säo funcoses definidas $e m U \times \mathbb{C}^{q}$

Demonstração Pelo Teorema Fundamental do Cálculo temos que

$$
\begin{aligned}
f\left(x_{1}, \ldots, x_{n}, y_{1}, \ldots, y_{4}\right)-\int_{0}^{1} \frac{d f}{d t}\left(t x_{1}, \ldots, t x_{n}, y_{1}, \ldots, y_{q}\right) d t \\
=\int_{0}^{1} \sum_{i=1}^{n} x_{i} \frac{\partial f}{\partial x_{2}}\left(t x_{1}, \ldots, t x_{n}, y_{1}, \ldots, y_{q}\right) d t \\
\quad \sum_{i=1}^{n} x_{i} \int_{0}^{1} \frac{\partial f}{\partial x_{i}}\left(t x_{1}, \ldots, t x_{n}, y_{1}, \ldots, y_{q}\right) d t
\end{aligned}
$$

Comando $f_{i}\left(x_{1}, \ldots, x_{n}, y_{1}, \ldots, y_{q}\right)=\int_{0}^{1} \frac{\partial f}{\partial x_{i}}\left(t x_{1}, \ldots, t x_{n}, y_{1}, \ldots, y_{q}\right) d t$ obtemos o resultado.

Lema 1.4 .2 (Lema de Nakayama) Sejam R um anel comulativo com elemento unidade I e $\mathcal{M}$ um ideal de $R$ com a propricdade que $1+x$ é intertivel em $R$ para todo $x \in \mathcal{M}$. Sejam $M$ um R-módulo e $A$ e $B R$-submódulos com A finitamenle gerado. Se $A \subseteq B+\mathcal{M} . A$ então $A \subseteq B$.

Demonstração Sejam $a_{1}, \ldots, a_{t}$ geradores de $A$. P'or hipótese, podemos encontrar $b_{1}, \ldots, b_{t}$ em $B$ e elenentos $\lambda_{i j}$ em $\mathcal{M}$ tais que para $1 \leq i<t$ temos

$$
a_{i}=b_{i}+\lambda_{i 1} a_{1}+\cdots+\lambda_{i l} a_{i}
$$




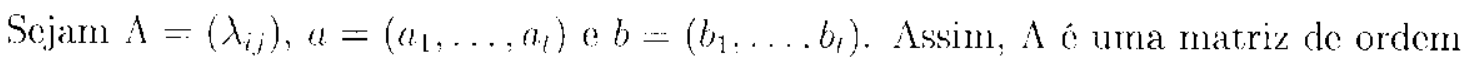
l. cujos elementos estào cm $R$ e a.b $\underbrace{M \times \ldots \times M}_{t \text { vors }}$. Fintäo podenos escrever (1.1) na format

$$
(1-A) a=b
$$

onde $I$ a a matriz identidade de ordem $/$ sobre $R$. Notemes que det $(I-\Lambda)-1-\lambda$, unde $\lambda$ é uma soma de produtos de elementos em $\mathcal{M}$ e, portanto é um elemento de $\mathcal{M}$. Por hipótese, $1-\lambda$ é invertivel em $h$. Portanto a matriz $I-\Lambda$ é invertível. $A$ ssim, podemos resolver o sistema (1.2) para $a_{1}, \ldots$ a $a_{t}$ en termos de $b_{1}, \ldots, b_{t}$. Segue que $a_{1}, \ldots, a_{t} \in B$. P'ortanto $A \subseteq B$.

O resultado seguinte é uma consequência do Lema de Nakidyama e sua demonstração pode ser encontrada enn [11].

Corolário 1.4.3 Sejam $R$ um anel local comutativo com identidade, $M$ seu ideal maximal

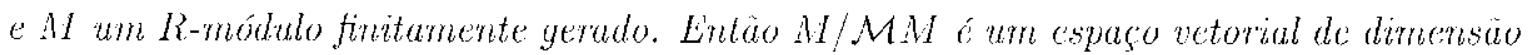

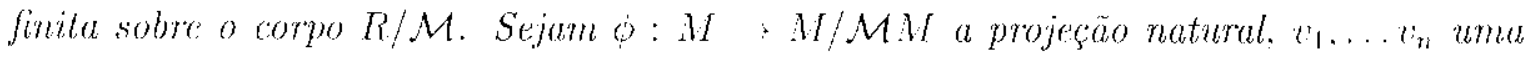
base para o espaço velorial $M / \mathcal{M M}$ e $e_{1}, \ldots e_{n} \in M$ tais que $\phi\left(e_{i}\right)=v_{i}$. Lntäo $e_{1} \ldots e_{n}$ formam un conjunlo de geradores de $M$ sobre $R$.

Para obter uma forma normal para germes ff em $\mathcal{M}_{2} \mathcal{O}_{2,2}$ de corank 1 , o seguinte teorema será utilizado. Sua demonstraçăo pode ser encontrada em [11].

Teorema 1.4.4 (Teorema da Preparação de Weierstrass) Seja f uma funçäo unalatica a valores complexos definida numa vizinhança de () em $\mathbb{C}^{2}$ satisfazendo

1. $f(0, y)=y^{k} g(y)$, onde $(0, y) \in \mathbb{C}^{2}$ e y e uma funça analitica de uma variavel definida numa vizinhança de 0 em $\mathbb{C}$,

2. $g(0) \neq 0$.

Entäo caiste uma funçäo a valores complexos q definida em uma vizinhança de (0 en $\mathbb{C}^{2}$ e funcoges $\lambda_{0}, \ldots, \lambda_{k-1}$ a valores complexos definidas numa vizinhança de 0 em $\mathbb{C}$ hais que

i) $q f(x, y)=y^{k}+\sum_{i-1}^{k-1} \lambda_{i}(x) y^{i}$ para qualquer $(x, y)$ numa vizinhansca de 0 em $\mathbb{C}^{2}$,

ii) $q(0) \neq 0$. 
Teorema 1.4.5 (Teorema da Preparação de Malgrange Generalizado) Sejam $f \in$ $\mathcal{O}_{n, p}$ : $M$ un $\mathcal{O}_{n}$-mó́dulo finitanente gerado. Entäo $M$ é um $\mathcal{O}_{p}$-módulo (via $f^{*}$ ) finitamente gerado se, esomente se. $\frac{M}{f^{*} \mathcal{M}_{p} \cdot M}$ e um espaco vetorial complexo de dimensäo finila.

Demonstração Ver $|11|$.

\subsection{Equivalência de Contato}

Introduzimos nesta seçào una outra relação de equivalência entre germes $\left(\mathbb{C}^{n}, x\right) \rightarrow$ $\left(\mathbb{C}^{p}: y\right)$. Esta relação é chamada de equivalência de contato ou $\mathcal{K}$-equivalência.

Definiçāo 1.5.1 Dois germes $f_{1}:\left(\mathbb{C}^{n}: x_{1}\right) \rightarrow\left(\mathbb{C}^{p}, y_{1}\right)$ e $f_{2}:\left(\mathbb{C}^{n}, x_{2}\right) \rightarrow\left(\mathbb{C}^{n}: y_{2}\right)$ são ditos serem $\mathcal{K}$-cquinalentes, se existem germes de difeomorfismos $h:\left(\mathbb{C}^{n}, x_{1}\right) \rightarrow\left(\mathbb{C}^{n}, x_{2}\right)$ e $H:\left(\mathbb{C}^{n} \times \mathbb{C}^{p},\left(x_{1}, y_{1}\right)\right) \rightarrow\left(\mathbb{C}^{n} \times \mathbb{C}^{p},\left(x_{2}, y_{2}\right)\right)$ para os quats o diagroma

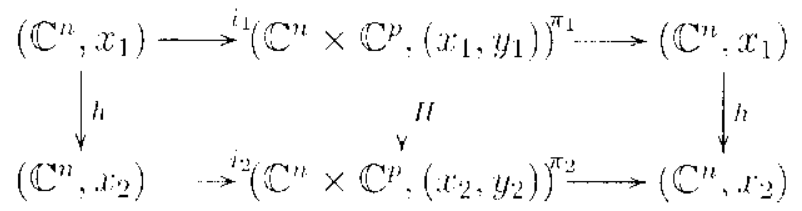

é comulativo, onde $i_{k}$ é o germe em $x_{k}$ da inclusäo $\mathbb{C}^{n} \rightarrow \mathbb{C}^{n} \times \mathbb{C}^{\mu}$ dada por $x \longmapsto\left(x, y_{k}\right)$ e $\pi_{k}$ é o germe em $\left(x_{k}, y_{k}\right)$ da projeçăo $\mathbb{C}^{n} \times \mathbb{C}^{p} \rightarrow \mathbb{C}^{n}$ dada por $(x, y) \longmapsto x, k=1,2$. Em outras palavrhs, $I I$ é dado por

$$
H(x, y)=(h(x), \theta(x, y))
$$

$\operatorname{com} \theta\left(x, y_{1}\right)=y_{2}$. Dizenos que $f_{1}$ e $f_{2}$ säo $\mathcal{K}$-equivalentes se existem $h$ e $H$ como acima lais que

$$
H\left(1, f_{1}\right)=\left(1, f_{2}\right) \circ h
$$

Dizemos que un germe $f^{\prime} \in \mathcal{O}_{n, p}$ in finitamente $\mathcal{K}$-determinado se existe $l \in \mathbb{N}$ satisfarzendo a seguinte condiçäo: para todo $g \in \mathcal{O}_{n, j}$ tal que $j^{l} g(0)$. $j^{l} f(0)$ temos que $g$ é $\mathcal{K}$-equivalente a $f$.

Se dois germes $f_{1}:\left(\mathbb{C}^{n}, x_{1}\right) \rightarrow\left(\mathbb{C}^{p}, y_{1}\right)$ e $f_{2}:\left(\mathbb{C}^{n}, x_{2}\right) \rightarrow\left(\mathbb{C}^{p}, y_{2}\right)$ sidu A-equivalentes. entäu também săo $\mathcal{K}$-equivalentes. De fato, suponhamos que existam germes do difeomorfismos $\emptyset:\left(\mathbb{C}^{n}, x_{1}\right) \rightarrow\left(\mathbb{C}^{n}, x_{2}\right)$ e $y:\left(\mathbb{C}^{p}, y_{1}\right) \rightarrow\left(\mathbb{C}^{p}, y_{2}\right)$ tais que

$$
f_{2} \circ 0=f_{1}
$$


Consideremos $H:\left(\mathbb{C}^{n} \times \mathbb{C}^{p},\left(x_{1}, y_{1}\right)\right) \rightarrow\left(\mathbb{C}^{n} \times \mathbb{C}^{\prime \prime},\left(x_{2}, y_{2}\right)\right)$ dado por $H(x, y)=(h(x), \theta(x, y))$, onde $h:\left(\mathbb{C}^{n}, x_{1}\right) \rightarrow\left(\mathbb{C}^{n}, x_{2}\right)$ o dado por $h(x)-\varphi(x)$ e $\theta:\left(\mathbb{C}^{n} \times \mathbb{C}^{p},\left(x_{1}, y_{1}\right)\right) \rightarrow\left(\mathbb{C}^{p}, y_{2}\right)$ c dado por $\theta(x, y)=\psi(y)$. Segue que

$\left.H \circ\left(1, f_{1}\right)(x)=\left(h(x), \theta\left(x, f_{1}(x)\right)\right)=\left(\phi(x), \varphi \circ f_{1}(x)\right)\right)=\left(\phi(x), f_{2} \circ \phi(x)\right)=\left(1, f_{2}\right) \circ h(x)$

Portanto $f_{1}$ e $f_{2}$ são $\mathcal{K}$-equivalentes. No cutanto, dois germes $\mathcal{K}$-equivalentes não são necessariamente $\mathcal{A}$-equivalentes.

Definição 1.5.2 Sejam $f_{1}$ e $f_{2}$ dois germes em $\mathcal{M}_{n} \mathcal{O}_{n . p}$. Dizemos que $f_{1}$ e $f_{2}$ säo $\mathcal{C}$ equivalentes se săo $\mathcal{K}$-equivalentes via $(H, h)$, onde h é o germe da identidade na origen.

Noternos que $f_{1}$ e $f_{2}$ sào $\mathcal{K}$-equivalentes se, e somente se, existe mu germe do difeomorfismo $h:\left(\mathbb{C}^{n}, 0\right) \rightarrow\left(\mathbb{C}^{n}: 0\right)$ tal que $f_{1} \circ h$ o $f_{2}$ säo $\mathcal{C}$-equivalentes.

Analogamente, dizemos que um germe $f \in \mathcal{M}_{n} \mathcal{O}_{n, p}$ e finitannente $\mathcal{C}$-determinado so existe $l \in \mathbb{N}$ satisfazendo a seguinte condição: para todo $g \in \mathcal{M}_{n} \mathcal{O}_{n, p}$ tal que $j^{\prime} g(0)=$ $j^{l} f(0)$ temos que $g$ é $\mathcal{C}$-equivalente a $f$.

O resultado seguinte é a Proposiçào 2.4 de $\mid 29]$

Proposição 1.5.3 Seja $\int \in \mathcal{O}_{n, p}$. Se $n \leq p e \int$ é finilamente $\mathcal{K}$-determinado entăo $f \hat{e}$ finitumente $\mathcal{C}$-determinado. 


\section{Capítulo 2}

\section{Anéis de Cohen-Macaulay}

\subsection{Resultados Básicos de Álgebra Comutativa}

Yesta seçào abordanos alguns resultados básicos de Álgebra Comutativa omitindo smas demonstraçoes. Tais resultados e suas demonstraçoes concontram-se em |1|: |19| e |20|.

Assumimos nesta seção que $R$ é um anel comutativo.

Definiçōes 2.1.1 Seja I um ideal de $R$.

1. O conjunto de todos os elementos $x \in R$ tais que alyuma potência de $x$ está em I é um ideal de $R$ chamado de ideal radical de $I$ e denotado por $\sqrt{I}$. Dizemos que $I$ é um ideal radical se $\sqrt{I}=I$.

2. Um elemento $x$ de $R$ é dito ser nilpotente se existe uma potência de $x$ que é igual a zero. O conjunto dos elementos nilpotentes de $R$ é denotado por nil $(R)$.

3. Dizemos que I é primário se $I \neq R$ ese

$$
x y \in I \text { entäo } x \in I \text { ou } y^{n} \in I \text { para algum } n>0
$$

O conjunto dos ideais primos de $R$ é chamado spectro de $R$ é denotado por spec( $R$ ).

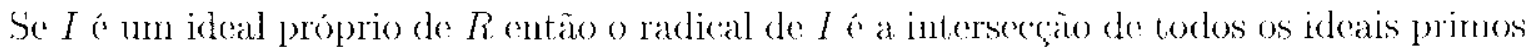
de $R$ clue contém $I$. Todo idleal primo é primário.

Se $R \neq 0$ então spec: $(R)$ tem elementos maximais e minimais e todo ideal primo de $R$ está contido em pelo menos um idcal primo naximal e contém pelo menos um ideal primo minimal.

Uma decomposição primária de um ideal $I$ de $R$ é uma expressäo de $I$ como uma 
intersecção finita de ideais primários

$$
I=\bigcap_{j=1}^{n} I_{j}
$$

Uma decomposição primária ó dita ser minimal se os ideais $\sqrt{I_{j}}$ são dois a dois distintos e

$$
I_{j} \not \bigcap_{k \neq j} I_{k}
$$

$1 \leq j \leq n$. Qualquer decomposiçào primária porde ser reduzida a uma decomposiçào primária minimal.

Nem todo ideal possui decomposiçäo printária e; quando a decomposição primária existe, nem sempre cla é única. Yo entanto, o seguinte resultado de |1, Corolário 4.11, pg 54 mostra que, sob certas condições, quando tal decomposição existe, então ela é única.

Teorema 2.1.2 Srjum I um ideal com decomposiçào primária minimal

$$
I=\bigcap_{i-1}^{n} I_{i}
$$

As componentes primárias $I_{2}$ de I tais que $\sqrt{I_{i}}$ co um primo minimal de I süo unicamente determinadas por I. Assim, se I tem uma decomposição primária minimal onde os radicais das componentes primárias süo primos minimais, entäo tal decomposiçáo á a anica com estas propriededer.

Definição 2.1.3 O anel $R$ é dito ser Noetheriano se para toda sequência ascendente de uldeais de $R$

$$
I_{1} \subseteq I_{2} \subseteq \ldots \subseteq \cdots
$$

criste $n$ tal que $I_{k}=I_{k+1}$ para lodo $k \geq n$.

Una formulação equivalente para a delinição acina é que um anel $R$ í Coetheriano se, e somente se, todo ideal do $R$ e finitamente gerado.

Como $\mathcal{O}_{n}$ ó isomorfo a $\mathbb{C}\left[X_{1}, \ldots, X_{n}\right.$, segue do Teorema da Base de Hilbert (ver $\left.\mid 1\right]$ ) que $\mathcal{O}_{n}$ é um anel Noetheriano.

O resultado seguinte, bem como sua demonstraçäo, podem ser encontrados ('nn [1]. Este resultado será utilizado no capítulo sceguinte.

Teorema 2.1.4 Em un anel Noetheriano todo ideal tem uma decomposiçüo primária. 
Definição 2.1.5 Seja $M$ um R-módulo. Dizemos que $M$ é Notheriano se para toda sequênuia ascendente de submódulos de $M$

$$
M_{1} \subseteq M_{2} \subseteq \ldots \subseteq \ldots
$$

eriste $n$ tal que $M_{k} \quad M_{k+1}$ parte todo $k \geq n$.

Un Ri-módulo $M$ é Noetheriano se e somente se, todo submodulo de $M$ é finitanente serado.

Se $R$ é um and Noethoriano o $M$ é um $R$-módulo finitamente gerado entäo $M e$ Noetheriano.

\subsection{Dimensão}

Nesta seção introduzimos o conceito de dimensäo de um módulo finitamente gerado, necessário para a definição de módulos de Cohen-Macaulay. Apresentamos também alguns resultados importantes omitindo as demonstrações. 'Tais resultados, bem como suas demonstrações, se encontran em Matsumura |19].

Consideremos $R$ un anel comutativo, $R \neq 0$.

Definição 2.2.1 Uma sequência de $n+1$ ideais primos da forma

$$
p_{0} \supset p_{1} \supset \ldots \supset p_{n}
$$

é chamada cadeia prima de comprimento $n$. Se $p \in$ sped $R$ ). o supremo de lodos os comprimentos das caderas primas com $p_{0}=p$ é chamado de altura de p e r demotado por $h t(p)$. Se I é um ideal próprio de $R$, definimos a altura de $I$ como sendo o infimo das alturas dos ideats primos de $R$ que contém $I$, ou seja,

$$
h t(I)=\inf \{h t(p) / p \supseteq I\}
$$

So ht $(p)$ - 0 então p é um primo minimal de $R$.

Definição 2.2.2 $A$ dimensão de $R$, lambém chamada de dimensão de Krull de $R$, é definida como semdo o supremo das alluras de lodos os ideais primos de $R$, ou seja,

$$
\operatorname{dim}_{k, r u l l} R \ldots \sup \{h(p) / p \in \operatorname{spec}(R)\}
$$

Também denotamos a dimensän de Krull de $R$ por dim $R$. 
Se $R$ é um domínio de ideais principais então $\operatorname{dim} R=1$.

Notemos que se a dimensão de $R$ é finita então é igual ao comprimento da mais longa cadeia prima em $R$.

Definição 2.2.3 Seja $M \neq 0$ um R-módulo. Definimos a dimensăo de $M$ como sendo

$$
\operatorname{dim} M=\operatorname{dim}(R / A m n(M))
$$

onde. Amn(M) $\quad\{a \in R / a M=0\}$. Congrnesonulmente, so $M=0$, dizenos que $\operatorname{dim} M=-1$.

() resultado seguinte o de grande importancia, pois tem como consequêneia imediatia que todo anel local Voctheriano tem dimensão finita. Sua demonstração é bastante extensa o pode ser encontradia cm $|19|$.

Teorema 2.2.4 Suponhamos que R seja Noetheriano e tenha um numero finito de ideais maxhais. Entäo a dimensäo de R. é finita.

Teorema 2.2.5 Suponhamos $R$ Noetheriano e seja $I=\left\langle a_{1}, \ldots, a_{r}\right\rangle$ um ideal de $R$ gerado por $r$ elcmentos. Se pé um ideal primo minimal de $\{p \in \operatorname{spec}(R) / p \supseteq I\}$, entäo $h(p) \leq r$. Em particular ha $(I)<r$.

\subsection{Grade e Anéis de Cohen-Macaulay}

As principais referencias para esta seção são $|2|$ e $|19|$.

Definição 2.3.1 Seja $M$ um R-mó́dulo. Una sequêneta $x_{1} \ldots, x_{n}$ de elementos de $R$ c chamada scquencia M regulat, ou M-sequencia, se $x_{i}$ nào a um divisor de zero cm $\frac{M}{r_{1} M+\cdots+x_{,} M}$ para $i=1, \ldots, n, \frac{M}{x_{1} M \cdots \cdots, x_{n, M}} \neq 0$.

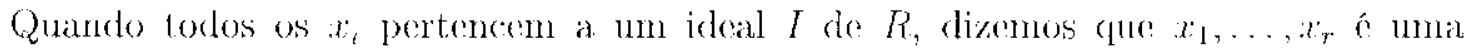
sequencia $M$-regular em $I$.

Definiçāo 2.3.2 Uma sequêneia $M$-regular $a_{1}, \ldots, x_{n}$ om um ideal $I$ de $R$ é maximal (respectivamente maximal em I) se $x_{1}, \ldots, x_{n-1}$ nìo é uma sequência $M$-regular para qualquer $x_{n+1} \in R$ (respectivamente $x_{n+1} \in I$ ).

Consideremos as sequências exatas curtas de $R$-módulos cue comogam cm $A$ e torminam em $B$

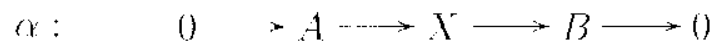




$$
\alpha^{\prime}: \quad 0 \cdot \cdots A \longrightarrow X^{\prime} \longrightarrow B \longrightarrow 0
$$

Dizemos que $\alpha$ é Yoneda equivalente a $\alpha^{\prime}$ se existe um homomorfismo $f: X \rightarrow X^{\prime}$ tal que o) segunte diagrama é comutativo

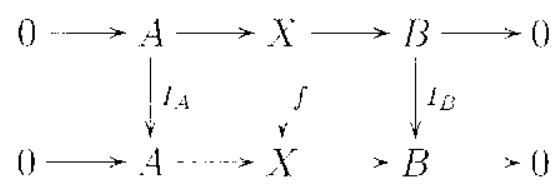

É possível mostrar que al equivalência Yoneda é uma relaçäo de cquivalência. Definimos Ext ${ }_{R}^{\prime}(B, A)$ como senclo o conjunto das classes de equivalencia das sequencias exalas curtas scgundo esta relação.

Consideremos agora as sequências exatas de $R$-módulos fue comegam em $A$ e terminam enr $B$ com $n$ módulus intemediários

$$
\begin{aligned}
& a: \quad 0 \cdots>A \quad>X_{1} \rightarrow>X_{2} \rightarrow>\cdots \rightarrow X_{n} \longrightarrow B \longrightarrow 0 \\
& \alpha^{\prime}: \quad 0 \longrightarrow A \longrightarrow X_{1}^{\prime} \longrightarrow X_{2}^{\prime} \longrightarrow \cdots \longrightarrow X_{n}^{\prime} \longrightarrow B \longrightarrow 0
\end{aligned}
$$

Dizemos que $\alpha$ e $\alpha^{\prime}$ são equivalentes se existem homomorfismos $f_{i}: X_{i} \rightarrow X_{i}^{\prime}, i=1, \ldots, n$, tais que o seguinte diagrama é comutativo

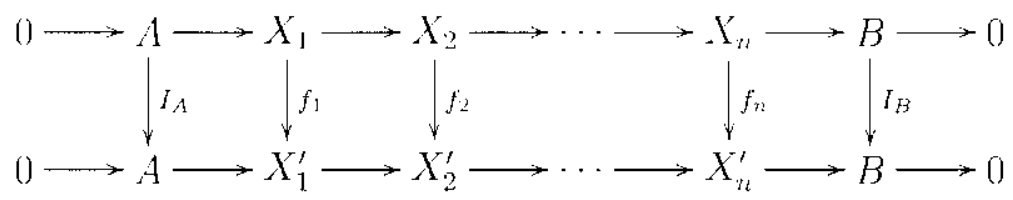

Esta relaçăo não é de equivalência, pois não é simétrica. Vo entanto, pordemos definir uma relaça de equivalencia a partir desta. Definimos entáo Ext $t_{R}^{\prime \prime}(B, A)$ como sendo o conjunto das classes de equivalencia das sequencias exatals cue comegan em $A$ e terminam em $B$ com $n$ módulos intermediários.

Teorema 2.3.3 /2, Teorema 1.9.5/ Suponhamos R Noetheriano e M um R-módulo finitamenle gerudo. Seja 1 um ideal de $R$ com $I M \neq M$. Entäo todas as sequências $M$-regulares maximais em 1 lem o mesmo comprimento n dado por

$$
n=\min \left\{i / \operatorname{Ext}_{R 2}^{i}(R / T, M) \neq 0\right\}
$$

Definição 2.3.4 Suponlanos $R$ Noetheriano $e M$ wm R-módulo finitamente: gerado. Seja I um ideal tal que $I M \neq M$. O grade de I cm $M$, denotado por grade $(I, M)$, ó 
o comprimento de todas as sequencias $M$-regulares maximais em $I$, ou seju,

$$
\text { grade }(T, M)=\min \left\{i / \operatorname{Lxt}_{R}(R / I, M) \neq 0\right\}
$$

Mostra-se que $I M=M$ se e somente se, grade $(I, M)=\infty$.

Definição 2.3.5 Suponhamos $R$ Noetheriano e $M \neq 0$ um $R$-módulo fintamente gerado. Entäo o grade de $M$ ć dado por

$$
\operatorname{grade} M=\min \left\{i / \operatorname{Ext}_{R}^{i}(M, R) \neq 0\right\}
$$

Se $M=0$ definimos grade $M \quad \infty$.

Se $I$ o um ideal de $R$ contäo grade $(R / I)$ grade $(I, R)$

Suponhamos $R$ local Noetheriano com ideal maximal $\mathcal{M}$ e $M$ um $R$-módulo finitamente gerado. Neste caso, o grade de $\mathcal{M}$ em $M$ é chamado de profundidade de $M$ e é denotado por depthM. Logo.

$$
\operatorname{depth} M=\min \left\{i / \operatorname{Ext}_{R}^{i}(R / \mathcal{M}, M) \neq 0\right\}
$$

O teorema seguinte á num resultado utilizado na definiçäo de anel de Cohen-Macaulay. Este resultado está demonstrado em |2|.

Proposição 2.3.6 Suponhamos $R$ local Noetheriano com ideal maximal $\mathcal{M}$ e $M$ um $R$ módulo finitumente gerado. Enläo

1. depth $M=\infty$ se, somente se, $M=0$.

2. Se $M \neq 0$ enträo depth $M<\operatorname{dim} M$.

Sejam $R$ um anel local Voetheriano. $\mathcal{M}$ sen ideal maximal e $M$ um $R$-módulo finitamente gerado. Vimos acima que depth $M \leq \operatorname{dim} M$ se $M \neq 0$.

Definição 2.3.7 Dizemos que $M$ á um módulo de. Cohen-Macaulay se $M$-.. 0 ou se $\operatorname{depth}(M)=\operatorname{dim} M . S e$ é ám R-módulo de Cohen-Macaulay, dizemos que $R$ é um anel de Cohen-Macualay.

\subsection{Módulos Projetivos e o Teorema de Hilbert-Burch}

As referências utilizadas nesta seçäo são $[2|| 3 \mid$, e $\mid 17]$.

Seja $R$ um anel comutativo. 
Definição 2.4.1 Dizemos que uma sequência exala de R-módulos

$$
0 \quad-H^{\prime} \stackrel{\leftrightarrow}{\longrightarrow} M \stackrel{\beta}{\longrightarrow} M^{\prime \prime} \longrightarrow 0
$$

cinde se $\beta$ lem inversa a direita, ou seja, existe um homomorfiono $\beta^{\prime}: M^{\prime \prime} \rightarrow M$ lal que $\beta \circ \beta^{\prime}=I_{M \prime \prime}$

O seguinte resultado, encontrado em |3|. será utilizado na aplicaçio do 'leorema de Hilbert-Burch.

Teorema 2.4.2 Se uma sequência exata de R-módulos

$$
0 \quad-M^{\prime}+M^{4}>M^{\prime \prime} \rightarrow 0
$$

cinde entäo $M^{\prime} \oplus M^{\prime \prime} \simeq M$

Definição 2.4.3 Dizenos que um R-múdulo $P$ éprojetino se dado umn epinorfismo de $R-m o ́ d u l o s ~ \sigma: B \rightarrow C$, cada aplicaçäo $\curvearrowright: P \rightarrow C$ tem um levantamento $B: P \rightarrow B$ tal que $\sigma \beta=\gamma$.

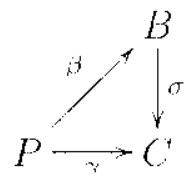

Proposição 2.4.4 /17, Proposiçäo 5.5/ Um R-módulo P'éprojetivo se, o somente se, é

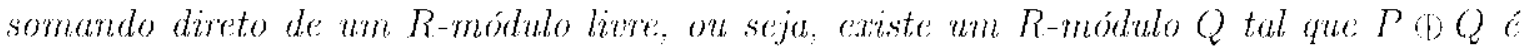
um R-módulo livre.

Teorema 2.4.5 Um R-módulo $P$ éprojetivo se, e somente se, $F_{x} t_{R}^{1}(P, G)=0$ para todo R-módulo G.

Definição 2.4.6 Seja C um R-módulo. Uma resoluçäo de $C$ é uma sequência exata de R-módulos e homomorfismos

$$
\longrightarrow X_{n} \stackrel{\delta}{\longrightarrow} X_{n-1} \stackrel{\delta}{\longrightarrow} \cdots \longrightarrow X_{1} \stackrel{\delta}{-}>X_{0} \stackrel{\bullet}{\longrightarrow} C \longrightarrow 0
$$

O teorema a seguir é um importante resultado cuja demonstraçäo se encontra em |2|.

Teorema 2.4.7 (Teorema de IIilbert-Burch) Srjum $R$ um anel local Noetheriano, $\varphi: R^{n} \rightarrow R^{n+1}$ uma aplicacào $R$-linear com matriz $M$ e $I_{n}(M)$ o ideal gerado pelos menores de ondem $n$ de $M$. Se grade $\left(R / I_{n}(M)\right) \geq 2$ entio $l_{n}(M)$ ten resoluça

$$
0 \longrightarrow R^{n} \stackrel{\hat{r}}{\longrightarrow} R^{n+1} \longrightarrow I_{n}(M) \quad \rightarrow 0
$$


O resultado seguinte é uma aplicação do teorema anterior para o ancl $\mathcal{O}_{2}$ e será utilizado no capítulos 4.

Corolário 2.4.8 Seja $\varphi: \mathcal{O}_{2}{ }^{\prime \prime} \rightarrow \mathcal{O}_{2}{ }^{n+1}$ uma aplicaçäo $\mathcal{O}_{2}$-linear com matriz $M$ e $I_{n}(M)$ o ideal gerado pelos menores de ordem $n$ de $M$. Se $\mathcal{O}_{2} / I_{n}(M)$ a um espaco vetorial de dimensia finita entüo a sequencia

$$
0 \longrightarrow \mathcal{O}_{2}{ }^{n}-\stackrel{r}{\rightarrow} \rightarrow \mathcal{O}_{2}{ }^{n+1} \quad>I_{n}(M) \quad>0
$$

é exala.

Demonstração Pelo teorema acima basta mostrar que grade $\left(\mathcal{O}_{2} / T_{n}(M)\right) \geq 2$. Consideremos a sequência cxata

$$
0 \quad \cdots I_{n}(M) \stackrel{i}{i} \rightarrow \mathrm{O}_{2}, \cdots{ }^{\pi} \rightarrow \underset{I_{n}}{\mathrm{O}_{2}}(M) \rightarrow 0
$$

onde $i$ c a aplicagào inclusào de $I_{n}(M)$ em $\mathcal{O}_{2}$ e $\pi$ c a projecão natural.

Mostremos primeiramente que a secpuencia (2.1) cinde. Sejam $m=\operatorname{dim}_{\mathrm{C}} \mathcal{O}_{2} / I_{n}(M)$ e $\left\{\bar{v}_{1}, \ldots, \bar{v}_{m}\right\}$ una base para $\mathcal{O}_{2} / I_{n}(M)$. Assim, dado $\bar{v} \in \mathcal{O}_{2} / I_{n}(M)$, tomos que $\bar{v}=$ $\alpha_{1} \bar{\nu}_{1}+\cdots+\alpha_{m} v_{m}$, onde $\alpha_{1}, \ldots, \alpha_{m} \in \mathbb{C}$ são mincamento determinados. Consideremos entião a aplicaşào linmar

$$
\begin{array}{rll}
\theta: \mathcal{O}_{2} / l_{n}(M) & \cdots & \mathcal{O}_{2} \\
\vartheta & \mapsto \alpha_{1} \eta_{1}+\ldots+\alpha_{m} v_{m}
\end{array}
$$

Como $\pi \circ \theta=1 d_{O_{2} / I_{n}(M)}$, rntão a sequencia 2.1 cinde.

Segue do Teorema 2.4 .2 que $\mathcal{O}_{2} \simeq I_{n}(M) \mathcal{O}_{2} / I_{n}(M)$. Cono $\mathcal{O}_{2}$ é um $\mathcal{O}_{2}$-módulo livre, pela Proposição 2.4 .4 temos que $\mathcal{O}_{2} / I_{n}(M)$ o mm $\mathcal{O}_{2}$-módulo projetivo e pelo Teorema 2.4 .5 lemos antiano que?

$$
\operatorname{Fixt}_{O_{2}}^{1}\left(\mathcal{O}_{2} / I_{n}(M), \mathcal{O}_{2}\right)=0
$$

Portanto

grade $\left(\mathcal{O}_{2} / I_{n}(M)\right)=\operatorname{grade}\left(I_{n}(M), \mathcal{O}_{2}\right)=\min \left\{i / \operatorname{Lxt}_{\mathcal{O}_{2}}^{i}\left(\mathcal{O}_{2} / I_{n}(M), \mathcal{O}_{2}\right) \neq 0\right\} \geq 2$ 


\section{Capítulo 3}

\section{Germes de Variedades Analíticas e Ideais de Fitting}

\subsection{Germes de Variedades Analíticas Complexas}

Vesta secăo introduzimos o concepto de germe de variedarle andítica complexa, germe de variedade de Cohen-Macaulay e apresentamos alguns resultados sobre vais germes. As

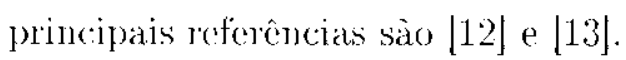

Definição 3.1.1 Uma subvariedade analítica complexa de um aberto $U \subset \mathbb{C}^{n}$ é um subconjunto $X$ de $U$ lal aue, em alguma vizinhança aberta $V$ de cada ponto de $U, X$ bo conjunto dos zeros de um número finito de funcöes analiticas definidas em $V$.

Neste trabalho, estamos interessados na estrutura de nma subvariedade muna vizinhança de un ponto fixade. Vannos considerar este ponte como sendo a origem. Consideremos os pares $\left(X_{r}, U_{n}\right)$, onde $U_{r}$ e uma vizinhança aberta da origem em $\mathbb{C}^{n}$ o $X_{n}$ ć uma subvariedade analítica de $U_{\alpha}$. Dois pares $\left(X_{\alpha}, V_{\alpha}\right)$ e $\left(X_{\beta}, U_{\beta}\right)$ säo equivalentes se existe

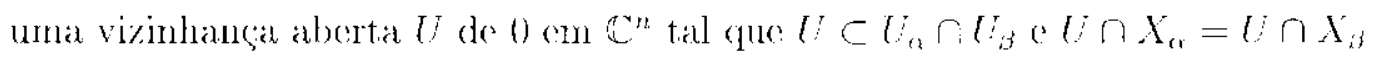

Definição 3.1.2 Un germe de uma subvariedade analítica complexa na origem om $\mathbb{C}^{\prime \prime}$ é

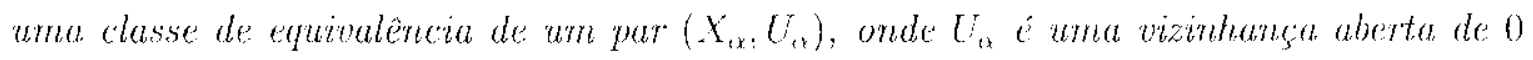
em $\mathbb{C}^{n}$ e $X_{6}$ é una suborariedade analática complexa de $U_{n}$.

Sejam $X_{1}$ e $X_{2}$ germes de subvariedades analíticas complexas em $\mathbb{C}^{n}$ e $\mathbb{C}^{p}$ respectivamente. Una aplicacào contínua $\varphi: X_{1} \rightarrow X_{2}$ é um germe na origem de uma aplicação contínua $\varphi$ definida num representante de $X_{1}$ com valores em um representante de $X_{2}$ e $\varphi(0) \quad 0$. Una aplicação contínua $\varphi: X_{1} \rightarrow X_{2}$ o dita ser mana aplicaça analítica complexa se os germes $Y_{1}$ e $X_{2}$ podem ser represcrntades por subvarjedades analíticas 
complexas de vizinhancas abertas da origem em $\mathbb{C}^{\prime \prime}$ e $\mathbb{C}^{p}$ respectivanonte, para as quais existe uma aplicaçäe analítica complexa $d: U_{1} \rightarrow U_{2}$ tal que $\Leftrightarrow\left(X_{1}\right) \subseteq X_{2}, \phi(0)=0$ o $\left.\varphi \quad \phi\right|_{X_{1}}$.

Ainda, estamos interessados nas propriedades des germes de subvariedades que independem de nudanças de coordenadas. Introduzimos entäo o conceito de germe de variedade analítica complexa como sendo uma conveniente classe de equivalencia de germes de subvariedades.

Definição 3.1.3 Sejum $X_{1}$ e $X_{2}$ genmes de subqatiedudes anulaticas completus. Dizemos que $X_{1}$ e $X_{2}$ säo equivalentes se existem aplicacous analiticas $\varphi: X_{1} \rightarrow X_{2}$ e $: X_{2} \rightarrow X_{1}$

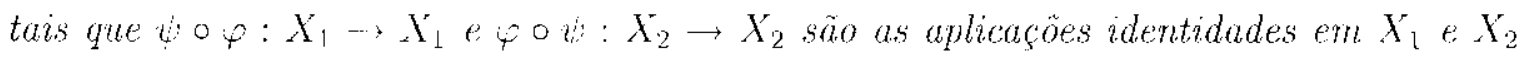
respectivamenté. Uma classe de equivalência é chamada de germe de variedadt analíticu complexa.

Lm germe de variedade analítica complexa sempre pode ser representado por $1 \mathrm{~m}$ germe de subvariedade andítica complexa en algum espaco vetorial complexo.

Dado $X$ um germe de subvariedade analítica complexa em $\mathbb{C}^{n}$, existe um ideal do anel local $\mathcal{O}$, consistindo dos germes de funçoes analíticas que se anulam em $X$. Esse ideal é chanado de ideal da subvariedade $X$ e e denotado por $I(X)$. Mais precisamente. $f \in I(X)$ se existe uma subvariedade $\vec{X}$ de um aberto $U$ que ó um representante de $X$ e una função analítica $\widetilde{f}: U \rightarrow \mathbb{C}$ que é um representante de $f$ tal que $\tilde{f}: \tilde{X} \equiv 0$.

Reciprocamente, dado un ideal $J$ de $\mathcal{O}_{n}$, existem $f_{1}, \ldots, f$, em $\mathcal{O}_{n}$ que geran $J$, pois $\mathcal{O}_{n}$ é Noetheriano. Sejann $\ell$ uma vizinhança aberta da origem e $\widetilde{f}_{1}, \ldots, \widetilde{f}_{r}$ representantes de $f_{1}, \ldots, f_{r}$ definidos em $U$. O subconjunto

$$
\left\{z \in U / \tilde{f}_{1}(z)=\ldots=\tilde{f}_{H}(z)=0\right\}
$$

define un germe de subvariedade analítica complexa que denotamos por $V(J)$.

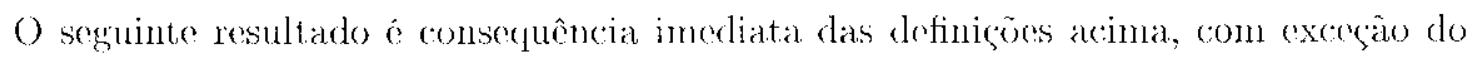
item (iv), que ć o conhecido T'eorema dos Zeros de Hilbert.

Proposição 3.1.4 Sujum $X, X_{1}$ e $X_{2}$ germes de subvariedades analiticas em $\mathbb{C}^{n}$ e. J, J e $J_{2}$ ideais de $\mathcal{O}_{n}$.

i) Se $X_{1} \subseteq X_{2}$, enläo $I\left(X_{1}\right) \supseteq I\left(X_{2}\right)$;

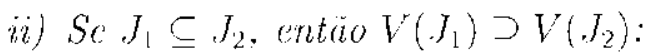

iii) $\mathrm{X}=V(I(X))$;

iv) $\sqrt{J}-I(V(J))$; 
v) $X_{1}=X_{2}$ st. somente se. $I\left(X_{1}\right)=I\left(X_{2}\right)$;

vi) $V\left(J_{1}+J_{2}\right)=V\left(J_{1}\right) \cap V\left(J_{2}\right)$ :

wii) $V\left(J_{1} \cap J_{2}\right)-V\left(J_{1}\right) \cup V\left(J_{2}\right)$.

Lim germe $X$ de uma subvariedade analítica ('m $\mathbb{C}^{n}$ e dito ser redutível se pode ser escrito como $X=X_{1} \cup X_{2}$, onde $X_{1}$ e $X_{2}$ sãe germes de subvariedades analíticas em $\mathbb{C}^{n}$ contidas propriamente en $X$. O germe $X$ é dito ser irredutível se näo é redutível.

Temos que $X$ ó irredutível se, e somente se, $I(X)$ e um ideal primo de $\mathcal{O}_{n}$ (ver $[14$, Toorema 13, pg 89). Além disso, toda subvariedade analítica complexa pode ser escrita como uma união finita de germes de subvariedades analíticas complexas irredutíveis. So a decomposição for tal que nenhum fator pode ser omitido, então ela é única e cada germe de subvariedade irredutível ó chamada de componente irredutível.

Sejann $X$ um germe de subvariedade analítica complexa e $I(X) \subseteq \mathcal{O}_{n}$. Como $\mathcal{O}_{n}$ é Noctheriano, $I(X)$ tem una decomposição primária:

$$
\left.I(X)=y_{1} \frown \ldots\right\urcorner y
$$

onde $q_{1}, \ldots, q_{r}$ sào ideais primários de $\mathcal{O}_{n}$. Logo

$$
X-V(I(X))-V\left(q_{1} \Gamma \ldots \Gamma q_{r}\right)-V\left(q_{1}\right) \cup \ldots \cup V\left(q_{r}\right)
$$

Consideremos $p_{i} \cdot \sqrt{q_{i}}, i=1, \ldots, n$. Como cada $q_{i}$ é primário, $p_{i}$ ó um ideal primo, $i=1, \ldots r$. Sabemos que $V\left(p_{i}\right)=V\left(q_{i}\right) \cdot \log 0 X=\cup_{i-1}^{r} V\left(p_{i}\right)$. Ainda $I\left(V\left(p_{i}\right)\right)-p_{i}$, que é prino, portanto cada $V\left(p_{i}\right)$ é irredutível. Lliminando os germes $V\left(p_{i}\right)$ tais que $V\left(p_{i}\right) \subseteq V\left(p_{j}\right)$ para algum $j \neq i$, obtemos entán a decomposicia em fatores irredutíveis de $X$ e cada $V\left(p_{i}\right)$ a uma componente irredutível de $X$.

\subsection{Anel $\mathcal{O}_{X, 0}$}

As principais referências para esta seção são [12] e [13|.

Seja $X$ un germe de subvaricdade analítica em $\mathbb{C}^{n}$. O anel dos germes de funçöes analíticas em $X$, denotado por $\mathcal{O}_{X, 0}$, é definido por

$$
\mathcal{O}_{X, i}=\frac{\mathcal{O}_{n}}{I(X)}
$$

Dado um germe $f \in \mathcal{O}_{n}$, seja $f$ um represcentante de $f$ definido numa vizinhança da origem. A restrição de $\tilde{f}$ a $X$ ó uma funçấo definicla numa vizinlança da origen contida. 
em $X$. 'Temos portanto um homomorfismo de $\mathcal{O}_{n}$ no anel dos germes de funçóes definidas em X. O núcleo deste homomorfismo o $I(X)$. Assim, $\mathcal{O}_{X, 0}$ pode ser identificado com o anel dos germes de funçoes analíticas definidas em $X$. Além disso, $\mathcal{O}_{X .0}$ a um and local Noetheriano.

() conjunto dos anéis $\mathcal{O}_{x, x}, x \in X$. forma um feixe de ancis que a denotado por $\mathcal{O}_{X}$. Informalmente $\mathcal{O}_{X}$ a um subeonjunto do conjunto dos germes de funçós contínuas definidas en $X$. Se $\pi: X \rightarrow Y$ ó una aplicação analítica entre gemes de subvariedades analíticas entäo $\pi$ definc um feixe de anéis $\pi_{*} \mathcal{O}_{X}$. Seus elementos podem ser pensados como germes do funçoes contímuas definidas em abertos da forma $\pi^{-1}(U)$, onde $U \subset Y$ é

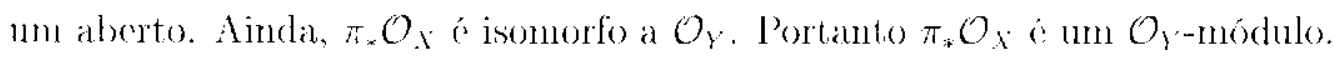

Sejam $X_{1}$ e $X_{2}$ germes de subvariedades analíticas complexas. Una aplicação analítica complexa $\varphi: X_{1} \rightarrow X_{2}$ induz um homomorlismo $\varphi^{*}: \mathcal{O}_{X_{2}, 0} \rightarrow \mathcal{O}_{X_{1}, 0}$.

Seja $X$ um germe de variedade complexa analítica irredutível. Definimos a dimensão de $X$ como sendo a dimensão de lirull do anel $\mathcal{O}_{X, 1}$. So $X$ é um genme de variedade analítica complexa qualquer en $\mathbb{C}^{n}$, cutão $X$ se escreve como mona união finita $\left.X=\right\lrcorner X_{i}$, onde cada $X_{i}$ é una variedade analítica complexa irredutível. Neste caso a dimensão de $X$ o dada por $\operatorname{dim} X \ldots \max _{i}\left\{\operatorname{dim} X_{i}\right\}$. O germe $X$ e dito ser equidimensional se dim $X$ dim $X_{i}$ parat todas as componentes $X_{i}$. A codimensão de $X$ é definida como sendo $n-\operatorname{dim} X$.

Definição 3.2.1 Seja X um germe de uma variedade analitica complexa. Dizemos que. $X$ é Cohen-Macaulay se o anel $\mathcal{O}_{X, 0}$ é um anel de Cohen-Macaulay, ou seja,

$$
\operatorname{depth} \mathcal{O}_{X, 0}=\operatorname{dim} \mathcal{O}_{X, 0}
$$

Definição 3.2.2 Una aplicaçüo analitica complexa $\chi: X_{1} \rightarrow X_{2}$ enble dois germes

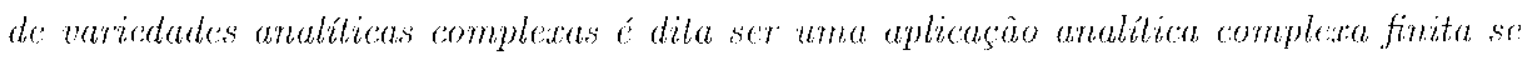
$\varphi^{-1}(0)=0$.

O resultardo seguinte é o Teorema $A_{1}$ de $|23|$.

Teorema 3.2.3 Sejam $X$ e $S$ gernes de variedades anuliticas complexas, $\pi: X \rightarrow S$

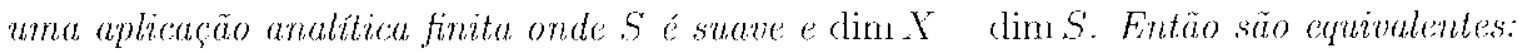

- X é Cohen-Macaulay.

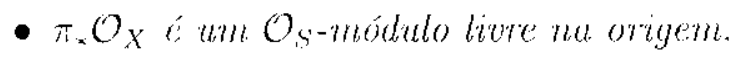

O resultado soguinte ó Teorema $A_{2}$ de [23]. 
Teorema 3.2.4 Sejam $\varphi$ uma matriz de ordem $r \times s$ som entradas em $\mathcal{O}_{n}$ e $Y_{k}(\varphi)$ o ideal gerado pelos menores de ordem $h$ de $\varphi$. Se $\left(0<k \leq s \leq r\right.$ entäo codim $V\left(I_{k}(\varphi)\right) \leq$ $(r-k-1)$. Se a igualdade vale, entäo $I_{k}(\varphi)$ define um germe de variedade do CohenMacaulay.

Teorema 3.2.5 Sega $\varphi: X_{1} \rightarrow X_{2}$ uma aplicacio analitica finita entre dois germes de variedades analuatas complexas onde $X_{1}$ é uma variedade de Cohen-Maraulay, $X_{2}$ é irredutivel e $\operatorname{dim} X_{1}=\operatorname{dim} X_{2} . S e \hat{\varphi}_{*} \mathcal{O}_{X_{1}}$ é um $\mathcal{O}_{X_{2}}$ módulo livre na origem entáo cada ponto num representante de $X_{2}$ possui

$$
\operatorname{dim}_{\odot} \frac{\mathcal{O}_{X_{1}, 0}}{\psi^{*}\left(\mathcal{M}_{X_{2}, 0}\right) \mathcal{O}_{X_{0}, 0}}
$$

pré imagens em $X_{1}$ contadas com multiplicidade, onde $\mathcal{M}_{\left.X_{2}, 1\right)}$ é o ideal matimal de $\mathcal{O}_{X_{2}, 0}$ e a dimensão é de espaco vetorial complexo.

Demonstraçāo Scgue de [3, Corolário 18.11] (ver comentário após o Corolário) que: toda variedade de Cohen-Macaulay é equidinensional. Assim, $X_{1}$ é equidimensionat. Se $X_{1}$ é equidimensional, $X_{2}$ é irredutível e $\operatorname{dim} X_{i}-\operatorname{dim} X_{2}$, então para todo ponto $p$ num representante de $X_{2}$ a cardinalidade de o ${ }^{l}(p)$ e constante, digamos, igual a $r$ (ver comentário na pg $25 \mathrm{~d}(13 \mid)$. Como $\varphi_{*} \mathcal{O}_{X_{1}}$ ó un $\mathcal{O}_{X_{2}}$-módulo livre na origem, segue do [13, Teorema $7, \mathrm{p}$, 25$]$ que

$$
r \ldots \operatorname{dim} c \frac{\mathcal{O}_{X_{1}, 0}}{\varphi^{*}\left(\mathcal{M}_{X_{2}, 0}\right) \mathcal{O}_{X_{1,0}}}
$$

\subsection{Normalização}

As principais referêneias para esta seça são |12| e |13|.

Definição 3.3.1 Seja $X$ um germe de variedade analitica complera. Dizemus que $X$ ć normal se o anel $\mathcal{O}_{X, 1}$ é igual ao seu fecho integral $\mathcal{O}_{X, 0}$.

Seja $X$ um germe de variedade analítica complexa irredutível. Lntäo o anel local $\mathcal{O}_{X, 0}$ é um domínio integral. Os elementos do corpo de fraçöes deste domínio integral săo chamados de germes de funçôes meromorfats em $X$. Assinn, unn germe de funçäo meromorfa $f$ é un germe da forma $h / g$, onde $h \in \mathcal{O}_{x, 0}$ e g $\subset \mathcal{O}_{X .0}-\{0\}$. Un germe de: aplicação é dito ser germe de aplicação meromorfa se cada una de suas componentes a un germe de função meromorfa. 
Uma normalização de $X$ ó um germe de variedade analítica complexa normal $\bar{X}$ e um germe de aplicaçào bimeromorfa $f:(\bar{X}, 0) \rightarrow(X, 0)$.

Suponhanos agora que $X$ seja redutível e seja $X=X_{1} \cup \ldots \cup X$, a decomposição de $X$ em suas componentes irredutíveis. Zma normalizacão $X$ de $X$ e definida como sendo a união disjunta de normalizaçoóes $\bar{X}_{i}$ das suas componentes.

\subsection{Intersecção Completa com Singularidade Isolada}

Definição 3.4.1 Sejam X um germe de variedade analitica complexa em $\mathbb{C}^{N}$ de dimensão ne I un ideal que define X. Dixemos que I define uma interseçäo completa en 0 se I admile $N-n$ geradores $\int_{1}, \ldots, \int_{N \cdots n}$ em $\mathcal{O}_{N}$.

Sejam $X$ uma variedade analítica em $\mathbb{C}^{N}$ de dimensio $n$ o $I(X)$ um ideal que define $X$. Suponhamos que $X$ seja uma interseção completa. Consideremos o caso em que $X$ tem uma singularidade isolada em 0 . Isto significa que se $f_{1}, \ldots, f_{N-n}$ é um conjunto de geradores de $I$, então existe uma vizinhança $V$ da origem em $C^{N}$ tal que $f_{1}, \ldots, f_{N}$ "convergem e para todo $y+0$ no conjunto dos zeros connuns de $f_{1} \ldots, f_{N-n}$, $d f_{1}(y), \ldots, d f_{N-n}(y)$ são lincarmente independentes. Teste caso dizomos que $X$ com sua C-álgebra local $\mathcal{O}_{N} / I$ é uma interseção completa com singularidade isolada ou, abreviadamente, ICIS. Frequentemente usamos expressón tais como

$$
\left(f_{1}, \ldots, f_{N-n}\right):\left(\mathbb{C}^{N}, 0\right) \rightarrow\left(\mathbb{C}^{N-n}, 0\right)
$$

$\left(\right.$ ou $I \subseteq \mathcal{O}_{N}$, ou $\left.\mathcal{O}_{N} / I\right)$ define um ICIS.

Definição 3.4.2 Seja $X$ uma interseçăo completa com singularidade isolada en 0 de dimensão n e $I=\left\langle f_{1}, \ldots, f_{k}\right\rangle$ urn ideal que define $X$. Definimos o número de Milnor de $X$ como sendo

$$
\begin{aligned}
& \sum_{i=1}^{k}(-1)^{k-i} \operatorname{dimc} \mathcal{O}_{n+k} /\left(\left\{\frac{\partial\left(f_{1}, \ldots, f_{k}\right)}{\partial\left(x_{j_{1}}, \ldots, x_{j_{k}}\right)}: 1 \leq j_{1}<\ldots<j_{k} \leq n-k\right\}, f_{1}, \ldots, f_{k}\right) \mathcal{O}_{n+k} \\
& \text { e denotamos por } \mu(X) \text { ou } \mu\left(f_{1}, \ldots, f_{k}\right) .
\end{aligned}
$$

\subsection{Ideais de Fitting}

Nesta seção definimos ideais do Fitting de um módulo $M$ e apresentamos um método para calcular tais ideais quando $M=\mathcal{O}_{n}$. As principais referencias são |23] e [22]. 
Definiçāo 3.5.1 Sejam $R$ um anel comutativo com unidade e M um R-módulo. Consideremos a sequênuia exata

$$
R^{p} \stackrel{\lambda}{\lambda} R^{q} \stackrel{\alpha}{\longrightarrow} M \longrightarrow 0
$$

Definimos o k-ésimo ideal de Fitting de $M$ como sendo o ideal em $R$ gerado pelos menores de ordem $(q-k)$ da matriz $\lambda$, onde $q-p \leq k<q$. Se $k \geq q$ definimos $\mathcal{F}_{k}(M)=R$ e se $k<q-p \operatorname{definimos} \mathcal{F}_{k}(M)-0$.

A definição de $\mathcal{F}_{k}(M)$ independe da escolha da sequência cxata (ver $[16,4$.D $\mid$ ).

Seja $f:\left(\mathbb{C}^{n}, 0\right) \rightarrow\left(\mathbb{C}^{n \cdot 1}, 0\right)$ um germe de aplicação holomorfa finitamente determinado. Para calcular o k-ésimo ideal de litcing de $f_{*} \mathcal{O}_{n}$ construímos uma secpuencia exata de $\mathcal{O}_{n+1}$-módulos

$$
\mathcal{O}_{n+1}^{p} \stackrel{\lambda}{\rightarrow} \rightarrow \mathcal{O}_{n+1}^{q} \stackrel{a}{\longrightarrow} f_{*} \mathcal{O}_{n} \longrightarrow 0
$$

onde o homomorfismo a leva os elementos da base de $\mathcal{O}_{r+1}^{q}$ nos geradores $g_{1}, \ldots, g_{l}$ de $f_{*} \mathcal{O}_{n}$ como $\mathcal{O}_{n-1}$-módulo e as colunas de $\lambda$ săo as relaçöes entre os $g_{i}$ com coeficientes em $\mathcal{O}_{n+1}$. Portanto $\mathcal{F}_{k}=\mathcal{F}_{k}\left(f_{*} \mathcal{O}_{n}\right)$ co o ideal de $\mathcal{O}_{n+1}$ gerado pelos menores de ordem $(q-k)$ de $\lambda$.

Segue do Corolário 1.4.3 que so as classes de $g_{1}, \ldots, g_{t}$ em $\frac{f_{*} \mathcal{O}_{n}}{\mathcal{M}_{n+1} f_{*} \mathcal{O}_{n}}$ geram $\frac{f_{*} \mathcal{O}_{n}}{\mathcal{M}_{n+1} f_{*} \mathcal{O}_{n}}$ como espaço vetorial sobre $\mathbb{C}$ então $g_{1}, \ldots g t$ geram $f_{*} \mathcal{O}_{n}$ como $\mathcal{O}_{n+1}$-módulo. Assim, para deceminar os geradores de $f_{*} \mathcal{O}_{n}$, basta determinar umal base de $\frac{f_{*} \mathcal{O}_{n}}{\mathcal{M}_{n+1} f_{*} \mathcal{O}_{n}}$. Se $g_{1}, \ldots, g l$

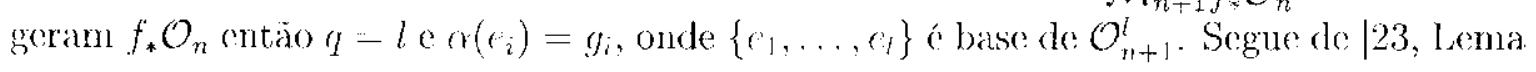
2.1) que existe uma sequencia exata como (3.2) onde $\lambda$ ć injetiva e, neste caso $p=: q$. 


\section{Capítulo 4}

\section{Cúspides e Nós de Germes de $\mathbb{C}^{2}$ em $\mathbb{C}^{2}$}

Em 1955 Whitncy (|30]) obteve formas normais para gemes estaveis de $\mathbb{C}^{2}$ em $\mathbb{C}^{2}$, que săo:

1. $(x, y) \rightarrow(x, y)$ (imersão)

2. $(x, y) \rightarrow\left(x, y^{2}\right)($ dobra $a)$

3. $(x, y) \rightarrow\left(x, x y+y^{3}\right)$ (cúspide de Whitney)

So considerarmos também multigermes, devemos acrescentar o bigerme

4. $\left\{\begin{array}{l}(x, y) \rightarrow\left(x, y^{2}\right) \\ \left(x^{\prime}, y^{\prime}\right) \rightarrow\left(x^{\prime 2}, y^{\prime}\right)\end{array}\right.$ (dobra dupla)

Definição 4.0.2 Seja $f \in \mathcal{M}_{2} \mathcal{O}_{2,2}$. Chamamos de conjunto singular de $f$, e denotamos por $S(f)$, o conjunto

$$
S(f):=\left\{x \in\left(\mathbb{C}^{2}, 0\right) / \operatorname{det}\left[l_{x} f\right]=0\right\}
$$

$O$ conjunto $\Delta(f)=\int(S(f))$ é chamado de discriminanle de $f$.

Seja $f \in \mathcal{M}_{2} \mathcal{O}_{2,2}$ um germe estável. Então $f$ é cquivalente a um gerne da forma (1), (2), (3) oll (4).

i) Se $f(x, y)=(x, y)$, critro $S(f)=\Delta(f)=\emptyset$.

ii) Suponhamos $f(x: y)=\left(x, y^{2}\right)$. Entăo

$$
\begin{gathered}
S(f) \cdots\left\{(x, y) \in\left(\mathbb{C}^{2}, 0\right) / y=0\right\} \\
\Delta(f)=\left\{f(x, 0) /(x, 0) \in\left(\mathbb{C}^{2}, 0\right)\right\}=\{(x, 0) / x \in(\mathbb{C}, 0)\}
\end{gathered}
$$


iii) Suponhamos $f(x, y)=\left(x, x y+y^{3}\right)$. Então

$$
\begin{gathered}
S(f) \quad\left\{(x, y) \in\left(\mathbb{C}^{2}, 0\right) / x+3 y^{2}=0\right\} \\
\Delta(f)=\left\{f\left(-3 y^{2}: y\right) / y \in(\mathbb{C}, 0)\right\}=\left\{\left(-3 y^{2},-2 y^{3}\right) / y \in(\mathbb{C}, 0)\right\} \\
=\left\{(x, y) \in\left(\mathbb{C}^{2}: 0\right) / 4 x^{3}+27 y^{2}=0\right\}
\end{gathered}
$$

que ce a cúspide ordinária.

iv) Suponhamos que $f$ é o bigerne $\left\{\begin{array}{lll}(x, y) & \rightarrow & \left(x, y^{2}\right) \\ \left(x^{\prime}, y^{\prime}\right) & \rightarrow & \left(x^{2} \cdot y^{\prime}\right)\end{array}\right.$. Fntão

$$
\begin{gathered}
S(f)=\left\{(x, y) \in\left(\mathbb{C}^{2}, 0\right) / y=0\right\} \cup\left\{\left(x^{\prime}, y^{\prime}\right) \in\left(\mathbb{C}^{2}, 0\right) / x^{\prime}=0\right\} \\
\Delta(f)=\{(x, 0) / x \in(\mathbb{C}, 0)\} \cup\left\{\left(0, y^{\prime}\right) / y^{\prime} \in(\mathbb{C}, 0)\right\}-\left\{(x, y) \in\left(\mathbb{C}^{2}, 0\right) / x y-0\right\}
\end{gathered}
$$

que ć unn nó simples.

Assim, se $f \in \mathcal{M}_{2} \mathcal{O}_{2.2}$ é un germe estável, as singularidades do $\Delta(f)$ correspondem a pontos (respectivamente pares de pontos) en $S(f)$ onde $f$ o um germe (respectivamente um bigerme) do tipo (3) (respectivanente (1)). Quando $f$ é do tipo (3), temos uma cúspide ordinária em $\Delta(f)$ e quando $f$ é do tipo (4), temos un nó em $\Delta(f)$. Se $f$ é como em (1) ou (2), mião $\Delta(f)$ é suave.

Quando un germe näo estável $f \in \mathcal{M}_{2} \mathcal{O}_{2,2}$ e perturbado de modo que se torne estável, um número de cúspides e nós aparece em seu discriminante. Denotamos o número de císpides por off) e o número de nós por $d(f)$. Os números e $f$ ) e d(f) refletem a complexidade do germe inicial. Neste capítulo, apresentamos fómulas para calcular e(f) e $d\left(\int\right)$.

\subsection{O invariante delta $(\delta)$}

Definiçāo 4.1.1 Um germe de uma variedade analatica complexa é dilo ser uma curva unalitica se é equidimensional e tem dimensào 1.

Seja $X$ um germe de curva analítica complexa en $\mathbb{C}^{n}$. Então $\mathcal{O}_{X, 0}-\frac{\mathcal{O}_{u}}{I(X)}$ tem dimensăo de Krull igual a 1 . Ainda, $\mathcal{O}_{n}$ é Noetheriano, $\log 0 I(X) \subseteq \mathcal{O}_{n}$ tem una decomposição primárià.

Definiçāo 4.1.2 Dizernos que um anel é reduzido ste séu único elemento nilpotente é o. 1sto é equivalente a dizer que $\langle 0\rangle$ é uma intersecçuo de ideais primos. 
Definição 4.1.3 Dizemos que uma curva analalica $X$ é reduzida se o anel $\mathcal{O}_{X, 1)}$ é um anel reduzido.

Seja $X$ uma curva analítica reduzida. Suponhamos que $X=\cup X_{i}$ seja sua decomposição em componentes irredutíveis. Para cadia $i$ s scjar $p_{i}$ un ideal primo de $\mathcal{O}_{n}$ tal que $V\left(p_{i}\right)=X_{i}$. Como $X$ é equidimensional, $\operatorname{dim} X-\operatorname{dim} X_{i}$ para todo $i$. Ainda, como cada $p_{i}$ é primo, $I\left(X_{i}\right)=\sqrt{p_{i}}=p_{i}$ para todo $i$. Portanto

$$
\operatorname{dim}_{\text {Krall }} \frac{\mathcal{O}_{n}}{p_{i}}=\operatorname{dim} X_{i}=1
$$

Consideremos agora o caso particular em que $X$ é uma curva plana. Neste caso, tomos o seguinte resultado de [12].

Teorema 4.1.4 Seja $X$ um germe de uma subvariedade analítica em $\mathbb{C}^{n}$. O germe $X$ é equidimensional de dimensäo $n-1$ se, e somente se, $I(X)$ é um ideal principal.

Assim, se Xé uma curva plana, $l(X)$ c um ideal principal. Seja $f \in \mathcal{O}_{n}$ tal que $I(X)=$ $\left\langle\int\right\rangle$. Como $\mathcal{O}_{n}$ é um domínio de fatoraçino única existem $f_{1}, \ldots, f_{n} \in \mathcal{O}_{n}$ irredutíveis tais que $f=f_{1}^{n_{1}} \ldots f_{r}^{n_{r}}$. Fintĩo

$$
\langle f\rangle-\left\langle\int_{1}^{n_{1}}\right\rangle \cap \ldots \cap\left\langle\int_{r}^{n_{r}}\right\rangle
$$

Como cada $f_{i}$ é irredutível entăo $\left\langle f_{i}^{n_{n}}\right\rangle$ é primário. De fato, sejam $g, h \in \mathcal{O}_{n}$ tais que $g h \in\left\langle f_{i}^{n_{i}}\right\rangle$ og $\notin\left\langle f_{i}^{n_{i}}\right\rangle$. Lntão $\int_{i}^{n_{i}}$ divide $g h$ e não divide $g$. Como $f_{i}$ é irredutível, então $f_{i}$ divide $h$. Portanto $h^{n_{2}} \in\left\langle f_{i}^{n_{2}}\right\rangle$, o que mostran que $\left\langle f_{i}^{n_{i}}\right\rangle$ o um ideal primário. Alóm disso, $\sqrt{\left\langle f_{i}^{n_{i}}\right\rangle}=\left\langle f_{i}\right\rangle$. Assim, a expressão (1.1) c uma decomposição primária de $f$.

Segue tamborm do fato de $f_{i}$ ser irredutivel que $\left\langle f_{i}\right\rangle$ é um primo mirimal para $i=$ $1, \ldots, r$.

Se $X$ é reduzida, $I(X)=\langle f\rangle$ é uma interseceão de ideais primos (que também são principais):

$$
\left.\langle f\rangle=\left\langle g_{1}\right\rangle\right\urcorner \ldots \cap\left\langle g_{s}\right\rangle
$$

A equação (4.2) é uma decomposição primária onde cada componente é um primo minimal. Scgue da unicidade da decomposição primária (Teorema 2.1.2) que $r=s$ e $\left\langle f_{i}^{n_{i}}\right\rangle=\left\langle g_{i}\right\rangle$, $i \ldots 1, \ldots, r$. Portanto $n_{i}-1, i-1, \ldots, r$.

lemos então que $p_{i}=\left\langle\int_{i}\right\rangle, i=1 \ldots r$ e

$$
\operatorname{dim} X_{i}=: \operatorname{dim} \text { krult }_{n} \frac{\mathcal{O}_{n}}{\left\langle f_{i}\right\rangle}
$$


Assim, uma curva analítica plana reduzida é dada por $f=f_{1} \ldots f_{r}$, onde $f_{i} \in \mathcal{O}_{n}$ é irredutível, $i=1, \ldots, r$.

Definição 4.1.5 S'eja $X$ um germe de urna curva analitica plana redazida. Entäo o

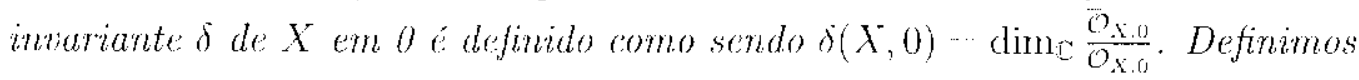

$$
\delta(X) \cdots \sum_{x \in X} \delta(X, x)
$$

Observação 4.1.6 Segue do $|27,3.2|$ que $\frac{\bar{O} x, 0}{\mathcal{O}_{x, 0}}$ e um espaço de dimensão finita e que a soma do lado direito na definiçào acima é finica, pois $\delta$ a não nulo ajemas nos pontos singulares. Assim, $\delta(X)$ o sempre finito.

Seja $X$ una curva analítica plana reduzida. Para calcular o invarianto $\delta(X, 0)$ usamos a fómula de Milnor, tendo en vista que o cálculo segundo a definição acima é difícil. Esta fórmula envolve o número de ramos de $X$ e o número de Milnor de $X$. O número de Milnor de $f \in \mathcal{O}_{n}$, denotado por $\mu(f)$, on simplesmente $\mu$, é a dimensão do espaço vetorial complexo

$$
\frac{\mathcal{O}_{n}}{\left\langle f_{x:}^{\prime} f_{y}\right\rangle}
$$

onde $f_{r}$ e $f_{y}$ sĩo as derivadas parciais de $f$. Como $I(X) \quad\langle f\rangle$ para algum $f \in \mathcal{O}_{n}$, o número de Milnor de $X$ é definido como sendo o número de Minor de f. A fórmula de Milnor (ver |21|) é dada por

$$
\mu-2 \delta-r+1
$$

onde $r$ é o número de ramos de $X$.

Observações 4.1.7 1 . Se $X$ é uma curva plana regular, entào $r=1$. De fato, segne do Teorema da Funça Implícita que, num ponto regular', uma curva plana é 1 m gráfico. Além disso, numa curva plana regular $\mu$ 0. Aplicando a fórmula de Milnor temos que $\delta=0$.

2. A fórmula de Milnor também mosira que $\delta$ é invariante por mudança de coordenadas.

\subsection{Cálculo de $c(f)$ e $d(f)$}

Lima consequência do Teorema 1.3 .3 co o seguinte resultado.

Proposição 4.2.1 Um germe $f \in \mathcal{M}_{2} \mathcal{O}_{2.2}$ é finitamente determinado se, e somente se, 1. $S(f)$ é uma curva reduzida. 
2. $f: S(f) \rightarrow \Delta(f)$ é bijetora

Demonstração Suponhamos f finitamente determinadà.

1. Seja $x_{0} \in S(f)-\{0\}$. Segue do Teorema 1.3 .3 que o germe de $f$ em $x_{0}$ é estável. Logo $S(f)$ é uma variedade diferenciável de dimensão 1 (ver [11]). Portanto $S(f)$ é uma curva reduzida. De fato, como $h=$ det $d_{x} f$ define $S(f)$, temos que $x_{0}$ é um valor regular de $h$, ou seja, $\frac{\partial h}{\partial x}\left(x_{0}\right) \neq 0$ on $\frac{\partial h}{\partial y}\left(x_{0}\right) \neq 0$. Logo, he $\frac{\partial h}{\partial x}$ (ou he $\frac{\partial h}{\partial y}$ ) năo tem raiz em comum e, portanto, $S(f)$ e recluzida.

2. Seja $x \in S(f)-\{0\}$. Então o germe de $\left.f\right|_{S(f)}$ em $x$ năo tem singularidade (ver [11]). Logo é um germe de imersäo e, portanto, injetor. Segue de [27] que $\Delta(f)$ também é uma curva reduzida.

Seja $f \in \mathcal{M}_{2} \mathrm{O}_{2,2}$ um gerne finitamente determinado. Entiăo existe un desdobramento a um parametro $F:\left(\mathbb{Q}^{2} \times \mathbb{C}, 0\right) \rightarrow\left(\mathbb{C}^{2} \times \mathbb{C}, 0\right), F(x, y, l)=(f l(x, y): l)$, tal que $f_{l} \dot{e}$ estável para todo $t \neq 0$. A aplicação $f_{t}$ e chamada de perturbação estável de $f$. Assim, $\left\{f_{t}, t \in(\mathbb{C}, 0)\right\}$ o uma família de germes em $\mathcal{M}_{2} \mathcal{O}_{2,2}$ com $f_{0}=f$ e $f_{t}$ estável para $t \neq 0$. Logo, para $t \neq 0, f_{t}$ é equivalente a um dos germes (1), (2), (3) ou (4) apresentados na introdução deste capítulo.

Teorema 4.2.2 Sejam $f \in \mathcal{M}_{2} \mathcal{O}_{2,2}$ um germe finitamente determinado e $F:\left(\mathbb{C}^{2} \times\right.$ $\mathbb{C}, 0) \rightarrow\left(\mathbb{C}^{2} \times \mathbb{C}, 0\right), F(x, y, t)=\left(f_{i}(x, y), t\right)$, tal que $f_{t}$ é estánel para todo $t \neq 0$. Entáo, para $t \neq 0, S\left(f_{t}\right)$ é suave $e$, portanto, $S(F)$ tem uma singularidade isolada em $(0,0)$.

\section{Demonstração}

Temos que $S\left(f_{t}\right)$ è suave para todo $t f 0$.

Além disso,

$$
\begin{gathered}
S(F)=\left\{(x, y, t) \in\left(\mathbb{C}^{3}: 0\right), / \operatorname{det}\left[d_{(x, y, n} F\right] \ldots 0\right\} \\
-\cdots\left((x, y, t) \in\left(\mathbb{C}^{3}, 0\right), / \operatorname{det}\left[d_{(x, y)} f_{t}\right]=0\right\} \\
=\left\{(x, y, t) \in\left(\mathbb{C}^{3}, 0\right), /(x, y) \subset S(f,)\right\}
\end{gathered}
$$

Portanto, $S(F)$ tem uma singularidade isolada em $(0,0)$.

Sejam $f \in \mathcal{M}_{2} \mathcal{O}_{2.2}$ unn germe finitamente determinado e $F:\left(\mathbb{C}^{2} \times \mathbb{C}, 0\right) \rightarrow\left(\mathbb{C}^{2} \times \mathbb{C}, 0\right)$, $F(x, y, t)=\left(f_{t}(x, y), t\right)$, tal que $f_{t}$ c estável para todo $t \neq 0$. Como $S(F)$ tem uma singularidade isolada om (0, (0), segue de [13. Corolário 1 do Teorema 15, pg 97] que $S\left(F^{\prime}\right)$ é normal e de $\mid 7$, Teorema $0.8(1) \mid$ gue $S(F)$ é uma nomalização de $\Delta\left(F^{\prime}\right)$. 
l'ixemos $t \in(\mathbb{C}, 0)$. Sejam

$$
\begin{aligned}
& S(F)_{t} \quad\left\{(x, y) \in\left(\mathbb{C}^{2}, 0\right) /(x, y, t) \in S(F)\right\} \\
& \Delta(F)_{t}=\left\{(x, y) \in\left(\mathbb{C}^{2} .0\right) /(x, y, t) \in \Delta(F)\right\}
\end{aligned}
$$

Notemes que $\operatorname{det}\left[d_{(x, y, t)} F\right]=0$ se. e somente se, det $\left[d_{(x, y)} f_{t}\right]=0, \log \left(S(F)_{t}=S\left(f_{\ell}\right)\right.$. Assim, se

$$
\begin{aligned}
\pi_{s}: S(F) & \longrightarrow \mathbb{C} \\
(x, y, t) & \longrightarrow t
\end{aligned}
$$

então $\pi_{s}^{-1}(l)=S(F)_{l}=S\left(f_{l}\right)$. Aindla, $\Delta(F)_{l}=\left\{f_{l}(x, y) /(x, y) \in S\left(f_{l}\right)\right\}=\Delta\left(f_{l}\right)$. Logo, se

$$
\begin{aligned}
\pi_{\Delta}: \Delta(F) & \longrightarrow \mathbb{C} \\
(x, y, t) & \longmapsto t
\end{aligned}
$$

então $\pi_{\Delta}^{1}(t)-\Delta(F)_{t} \cdots \Delta\left(f_{t}\right)$.

Pela Proposição 3.3 [27| temos que?

$$
\delta\left(\Delta\left(f_{1}\right)\right)=\delta\left(\Delta\left(f_{0}\right)\right)-\delta\left(S\left(f_{0}\right)\right)
$$

Teorema 4.2.3 Sejam $f \in \mathcal{M}_{2} \mathrm{O}_{2,2}$ um germe estável e finitamente determinado e $\Delta(f)$ seu discriminante. Enlão $\delta(\Delta(f),(x, y))=0$ ou 1 para todo $(x, y) \in \Delta(f)$.

Demonstração Vimos na Proposição 4.2.1 que $\Delta(f)$ é uma curva reshuzida. Scja $\left(x_{1}, y_{1}\right) \in \Delta(f)$. Então $\left(x_{1}, y_{1}\right)=f\left(x_{0}, y_{0}\right)$ para algum $\left(x_{0}, y_{0}\right) \in S(f)$. Segne do Toorema 1.3 .3 que o germe de $f$ em $\left(x_{0}, y_{0}\right)$, que denotamos também por $f$, éstável, logo e equivalente a um germe da forma (1), (2), (3) ou (1).

Se $f$ é da forma (1) ou (2) cntão $\Delta(f)$ é regular e, portanto, $\delta=0$.

Suponhanos $f$ equivalente ao germe da forma (3). Neste caso $\Delta(f)$ tem equação $4 x^{3}+27 y^{2}-\ldots$ o. Seja $g(x, y)-4 x^{3}+27 y^{2}$. Entrão

$$
\mu(g)=\operatorname{dim} \frac{\mathcal{O}_{2}}{\left\langle\frac{\partial g}{\partial x} \frac{\partial g}{\partial y}\right\rangle}=\operatorname{din} \frac{\mathcal{O}_{2}}{\left\langle r^{2}, y\right\rangle}=2
$$

Comor $r \cdot 1$ e $\mu-2$ segue gue

$$
\delta=\frac{\mu+r-1}{2}=1
$$

Suponhamos $f$ equivalente ao germe da forma (1). Então $\Delta(f)$ tem equação $g(x, y)=-$ $x y=0$. Lintão

$$
\mu(g)=\operatorname{dim} c \frac{\mathcal{O}_{2}}{\left\langle\frac{\partial g}{\partial x}, \frac{\partial g}{\partial y}\right\rangle}=\operatorname{dim}_{\mathbb{C}} \frac{\mathcal{O}_{2}}{\langle x, y\rangle}=1
$$


Como $r=2$ e $\mu=1$ segue que

$$
\delta-\frac{\mu+r-1}{2}=1
$$

Como $\delta\left(\Delta\left(f_{i}\right)\right)=\sum_{(x, y) \in \Delta\left(f_{t}\right)} \delta\left(\Delta\left(f_{t}\right),(x, y)\right)$ entíno o lado escuerdo de (4.3) consiste das somas das contribuiçoons locais das singularidades de $\Delta\left(f_{t}\right)$ que são cúspides on nós. Cada cúspide contribui com 1 e cada nó contribui con 1.

As singularidades de $\Delta\left(f_{t}\right)$ correspondem a pontos (respectivamente pares de pontos) em $S\left(f_{t}\right)$ onde $f_{t}$ é un germe (respectivamente um bigerme) do tipo (3) (respectivamente (4)).

Se $c(f)$ é o núnero de cúspides e $d(f)$ é o número de nós em $\Delta\left(f_{t}\right)$, que tendem a 0 quando $t$ tencle a 0 , então temos por $(\cdot 1,3)$

$$
c(f)+d(f)=\delta\left(\Delta\left(f_{t}\right)\right)=\delta\left(\Delta\left(f_{0}\right)\right)-\delta\left(S\left(f_{0}\right)\right)
$$

Como conscyuencia da Proposição 4.2.1, de (1.1) e da ()bservação 4.1 .6 temos o seguinte resultado.

Proposição 4.2.4 Um germe $\int \in \mathcal{M}_{2} \mathrm{O}_{2,2}$ é finitamente determinado se, e somente se, $c(f)<\infty e d(f)<\infty$.

$A$ seguir, apresentamos un resultado de [23] que rolaciona e $(f)$ e $d(f)$ com ideais de Fitting. Sejam $X$ um germe de uma variedade $n$-dimensional, $X_{1}, \ldots, X_{m}$ as componentes irredutíveis de $X$ e $f:(X, 0) \rightarrow\left(\mathbb{C}^{2}, 0\right)$ analítica e finita. Segue de $[27,5.2 .10]$ que $f\left(X_{i}\right)$ é variedades. Temos então a decomposição em componentes irredutíveis

$$
f\left(X_{i}\right)=V\left(q_{i_{1}}\right) \cup \ldots \cup V\left(q_{i,}\right)
$$

como na seção 3.1.

Chamanos de imagem reduzida de $X_{i}$ por $f$ à variedade reduzida

$$
\tilde{X}_{i}=V\left(\sqrt{q_{i_{1}}}\right) \cup \ldots \cup V\left(\sqrt{q_{i}}\right)
$$

Teorema 4.2.5 Sejam $(X, 0)$ um germe de uma curva reduzida ef $f:(X, 0) \rightarrow\left(\mathbb{C}^{2}: 0\right)$ finita e injetiva. Se $\mathcal{F}_{1}=\mathcal{F}_{1}\left(f_{*} \mathcal{O}_{S(N)}\right)$ é o primeiro ideal de Fitting do $\mathcal{O}_{2}$-módulo $f_{*} \mathcal{O}_{S(f)}$, entio

$$
c(f)+d(f)-\operatorname{diml} \mathcal{O}_{2} / \mathcal{F}_{1}
$$

Demonstração Ver $\mid 23$, Teo 3.6|. 
Exemplo 4.2.6 Consideremos $f \in \mathcal{M}_{2} \mathcal{O}_{2,2}$ dadia por $f(x, y)=\left(x, y^{4}+x y\right)$. Temos que $f$ e um germe finitamente deterninado. Cono $S(f)$ tem dimensäo 1, ntilizamos a receita apresentada no capítulo 2 para calcular o ideal de Fitting de $f_{*} \mathcal{O}_{\text {sifj) }}$.emos

$$
\begin{aligned}
& S(f) \quad\left\{(x, y) \in\left(\mathbb{C}^{2}, 0\right) / 4 y^{3}+x=0\right\}=\left\{\left(-4 y^{3}, y\right) / y \in(\mathbb{C}, 0)\right\} \\
& f s_{S(f)}:(S(f), 0) \rightarrow\left(\mathbb{C}^{2}, 0\right) \\
&\left(-4 y^{3}, y\right) \longrightarrow\left(-4 y^{3},-3 y^{3}\right)
\end{aligned}
$$

$\log ()$

$$
\frac{f_{*} \mathcal{O}_{S(J)}}{\mathcal{M}_{2} f_{*} \mathcal{O}_{S(f)}} \simeq \frac{\mathcal{O}_{1}}{\left\langle-1 y^{3} \cdot-3 y^{4}\right\rangle} \simeq \mathbb{C}\left\{1, y, y^{2}\right\}
$$

Segue cutào do Corolírio 1.1 .3 que $\mathcal{O}_{S(f)}$ e gerado por $1, y, y^{2}$ sobre $\mathcal{O}_{22}$. Encontremos agora as relaçós entre $1, y e y^{2}$. Considerando $U=-4 y^{3}$ e $V=-3 y^{4}$ obtemos as relaçoes

$$
\begin{gathered}
U^{2} \cdot 1-0 . y+16 / 3 V \cdot y^{2}=0 \\
-4 V .1+3 U \cdot y+0 . y^{2} \quad 0 \\
0.1-4 V . y+3 U \cdot y^{2}=0
\end{gathered}
$$

$\log (0$

$$
\lambda=\left(\begin{array}{ccc}
U^{2} & 0 & 16 / 3 V \\
-4 V & 3 U & 0 \\
0 & \cdots 1 V & 3 U
\end{array}\right)
$$

c, portanto,

$$
\mathcal{F}_{1}\left(f_{*} \mathcal{O}_{S(f)}\right)=\left\langle U^{2}, V^{2}, U V, U^{3}, U^{2} V\right\rangle
$$

Segue que

$$
c(f)+d(f)=\operatorname{dim} \mathcal{O}_{2} /\left\langle U^{2}, V^{2}, U V, U^{3}, U^{2} V\right\rangle-3
$$

Denotiamos por $\Sigma_{f}^{i}$ o conjunto

$$
\left\{(x, y) \subset\left(\mathbb{C}^{2}, 0\right) / \operatorname{dim} \operatorname{ker} d(x, y) f=i\right\}
$$

e por $\sum_{f}^{i, 3}$ o conjunto

$$
\left\{(x, y) \in \Sigma_{f}^{i} /\left.\operatorname{dim} \operatorname{ker} d_{(x, y)} f\right|_{:_{j}^{i}}=j\right\}
$$

Proposição 4.2.7 Seja $f \subset \mathcal{M}_{2} \mathcal{O}_{2,2}$ lal que $\Sigma_{j}^{1.1} \quad\{0\}$. Entüo

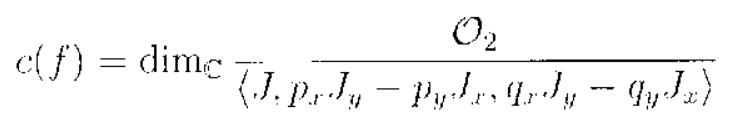

onde p e q sũo as componenles de $f, J$ é o determinante jacobiano de $f$ e os subscritos 
indicam derivadas parciais.

Demonstração (O ideal no denominador de (4.5) co o ideal $I_{2}\left(M_{f}\right)$ gerado pelos menores de ordem 2 da matri\%

$$
M_{f}=\left(\begin{array}{cc}
p_{x} & p_{y} \\
q_{x} & q_{y} \\
J_{x} & J_{y}
\end{array}\right)
$$

Temos que $V\left(I_{2}\left(M_{f}\right)\right)=\sum_{f}^{1,1}$. De fato, sejam $f: U \rightarrow \mathbb{C}^{2}$ um representante do germe $f$, ondo $U$ é uma virinhança aberta de zero em $\mathbb{C}^{2}, e\left(x_{0}, y_{0}\right) \in V\left(I_{2}\left(M_{f}\right)\right)$. Entào

$$
\begin{aligned}
& J\left(x_{0}, y_{0}\right)=\left(p_{x} q_{y}-p_{y} q_{x}\right)\left(x_{0}, y_{0}\right)=0 \\
& \left(p_{x} J_{y}-p_{y} J_{x}\right)\left(x_{0}, y_{0}\right)=0 \\
& \left(q_{x} J_{y}-q_{y} J_{x}\right)\left(x_{0}, y_{0}\right)=0
\end{aligned}
$$

Como $0 \in \Sigma_{f}^{1}$, então dim Innd $f=1$ e, portanto, uma das funç̧öes $p_{x}$ ou $p_{y}$ on $q_{x}$ ou $q_{y}$ não so anula em 0. Fazendo mudanças de coordenadas na fonte e na meta se necessário, podemos supor que $p_{x}(0) \neq 0$. Encolhendo $U$ se necessário, podemos também supor que $p_{x}(x, y) \neq 0$ para todo $(x, y) \in U$. Segue que para todo $(x, y) \in U$ tal que $J(x, y)=0$ temos $(x, y) \in \Sigma_{f}^{1}$. Logo

$$
\Sigma_{f}^{1}=\{(x, y) \in U / J(x, y)=0\}
$$

Assim $\left(x_{0}, y_{0}\right) \in \Sigma_{f}^{1}$. Calculemos então $d_{\left(x_{0}, y_{0}\right)} f f_{x_{f}}$. Temos que

$$
\left.d_{\left(x_{0}, y_{0}\right)} f\right|_{\Sigma_{f}^{-1}}=\left.\left(d_{\left(x_{1}, y_{1}\right)} f\right)\right|_{\left.T_{\left(x_{0}, y_{0}\right)}\right) P_{f}^{1}}: T_{\left(x_{0}: y_{0}\right)} \Sigma_{f}^{1} \rightarrow d_{\left(x_{0}, y_{0}\right)} f\left(T_{\left(x_{0}, y_{0}\right)} \Sigma_{j}^{1}\right)
$$

é dada por

$$
\left.d_{\left(x_{0}, y_{0}\right)} f\right|_{\Sigma_{f}^{1}}(u, v)=d_{\left(x_{n}, y_{1}\right)} f(u, v)=\left(p_{x}\left(x_{0}, y_{0}\right) u+p_{y}\left(x_{0}, y_{0}\right) v, q_{x}\left(x_{0}, y_{0}\right) u+q_{y}\left(x_{0}, y_{0}\right) v\right)
$$

Se $(u, v)$ é um vetor langente a $\Sigma_{f}^{1}$ em $\left(x_{0}, y_{0}\right)$ (nitão $(u, v)$ pertence ao núcleo da derivada de $J$, isto é, $d_{\left(x_{0}, y_{(j)}\right)} J\left(u, v^{\prime}\right)=0$. Nas

$$
d_{\left(x_{i}, y_{0}\right)} J(u, v)=J_{u}\left(x_{0}, y_{0}\right) u+J_{y}\left(x_{0}, y_{0}\right) u
$$

$\log 0, J_{x}\left(x_{0}, y_{0}\right) u+J_{y}\left(x_{0}, y_{0}\right) u=0$. Fintão

$$
\begin{gathered}
\left.\operatorname{ker} d_{\left(x_{0}, y_{0}\right)} f\right|_{\Sigma_{f}}\left\{(u, v) / J_{x}\left(x_{0}, y_{0}\right) u \cdot J_{y}\left(x_{0}, y_{0}\right) v-p_{x}\left(x_{0}, y_{0}\right) u+p_{y}\left(x_{0}, y_{0}\right) v\right. \\
\left.=y_{x}\left(x_{0}, y_{0}\right) u+q_{y}\left(x_{0}, y_{0}\right) v \cdots 0\right\}-\left\{(u, v) / M_{f}\left(x_{1}, y_{0}\right)(u, v)-0\right\}
\end{gathered}
$$


Por (4.6) temos que o rank de $M_{f}\left(x_{0}, y_{0}\right)$ e 1 , logo dim ker $\left.d_{\left(x_{0}, y_{0}\right)} f\right|_{\Sigma_{f}^{1}}=1$. Segue que $\left(x_{0}, y_{0}\right) \in \Sigma_{f}^{1,1}$. Como $\Sigma_{f}^{1,1}=\{0\}$, é imediato que $\Sigma_{f}^{1,1} \subset V\left(I_{2}\left(M_{f}\right)\right)$.

Consideremos agoral

$$
\begin{aligned}
F:\left(\mathbb{C}^{3}, 0\right) & \longrightarrow\left(\mathbb{C}^{3}, 0\right) \\
(x, y, u) & \longrightarrow(p(x, y, u), q(x, y, u), u)=(f,(x, y), u)
\end{aligned}
$$

uma deformaça a 1-parametro de $f$, onde $f_{u}$ o estável para todo $u \neq 0$. Sejam

$$
M_{k}=\left(\begin{array}{cc}
p_{x}(x, y, u) & p_{y}(x, y, u) \\
\psi_{x}(x, y, u) & q_{y}(x, y, u) \\
J_{x}(x, y, u) & J_{y}(x, y, u)
\end{array}\right)
$$

a $X=V\left(I_{2}\left(M_{F}\right)\right)$. Temos que $(x, y, u) \in X$ se, e somente se, $(x, y) \in \Sigma_{f}^{1,1}$. Fixemos $u \neq-\left(\right.$ ) Como $f_{u}$ ć estável, então $f_{u}$ ć uma imersão, uma dobra, uma císpide de Whitney on uma dobra dupla. Logo, $\sum_{f_{u}}^{1,1}$ o vazio, exceto quando $f_{u}$ é a cúspide de Whitncy e, neste caso, $\Sigma_{f_{n}}^{1, l}=\{(0,0)\}$.

Segue que $\Sigma_{f_{u}}^{1.1}$ tem dimensão 0 e, portanto, $X=\cup_{u} \sum_{f_{u}}^{l, 1}$ tem dimensão igual a 0 ou 1, ou seja, a codimensăo de $X$ é maior ou igual a 2. Pelo Teorema 3.2.4 temos que a codinensão de $X$ é igual a 2 e $X$ é uma variedade de Cohen-Macaulay.

Consideremos a projeccão lia base do desdobramento

$$
\begin{aligned}
\pi:(X, 0) & \longrightarrow(\mathbb{C}, 0) \\
(x, y, u) & \longmapsto u
\end{aligned}
$$

Temos que

$$
\pi^{-1}(0)=\{(x, y, 0) \in(X, 0)\}=\left\{(x, y, 0) /(x, y) \in \mathcal{\Sigma}_{j}^{1,1}\right\}=\{(0,0,0)\}
$$

Segue do Teorema 3.2 .3 que $\pi_{*} \mathcal{O}_{X}$ é um $\mathcal{O}_{c}$-módulo livre na origem. Segue (ntizo do Teorema 3.2 .5 que cada ponto $u$ em $(\mathbb{C}, 0)$ tem

$$
\operatorname{dim}_{C} \mathcal{O}_{X, 0} / \pi^{*}\left(\mathcal{M}_{\subset, 0}\right) \mathcal{O}_{X, 0}
$$

pré-indagens en $X$ contadas com multiplicidade.

Mostremos que

$$
\operatorname{dim}_{\mathbb{C}} \frac{\mathcal{O}_{X, 0}}{\pi^{*}\left(\mathcal{M}_{\mathbb{0}, 0}\right) \mathcal{O}_{X .0}}=\operatorname{dim}_{\mathbb{C}} \frac{\mathcal{O}_{2}}{I_{2}\left(M_{J}\right)}
$$

Sabemos que

$$
\mathcal{O}_{X 00} \simeq \frac{\mathcal{O}_{3}}{I_{2}\left(M_{r}\right)}
$$


Dada a aplicaça inclusão

$$
\begin{aligned}
& i: \mathbb{C}^{2} \longrightarrow \mathbb{C}^{3} \\
& (x, y) \longmapsto(x: y, 0)
\end{aligned}
$$

Temos o homomorfismo induzido

$$
\begin{aligned}
k^{*}: \mathrm{O}_{3} & \longrightarrow \mathrm{O}_{2} \\
k & \longmapsto k \circ i
\end{aligned}
$$

Dado $f \in I_{2}\left(M_{F}\right)$,

$$
i^{*}(f)(x, y)=f \circ i(x, y)=f(x, y, 0) \in I_{2}\left(M_{f}\right)
$$

Logo, $i^{*}\left(I_{2}\left(M_{F}\right)\right)<I_{2}\left(M_{f}\right)$. Assim $i^{*}$ induz um homomorfismo sobrejetor

$$
i^{*}: \frac{\mathcal{O}_{3}}{I_{2}\left(M_{F}\right)} \rightarrow \frac{\mathcal{O}_{2}}{I_{2}\left(M_{f}\right)}
$$

que chamaremos também de $i^{x}$. 'lemos que

$$
\operatorname{ker} i^{*}=\left\{\bar{g} \in \underset{I_{2}\left(M_{j}\right)}{\mathcal{O}_{3}} / i^{*}(g) \in I_{2}\left(M_{j}\right)\right\}
$$

Dado $g \in \mathrm{ker} i^{*}$, temos $g(x, y, 0) \in I_{2}\left(M_{f}\right)$ para todo $(x, y) \in\left(\mathbb{C}^{2}, 0\right)$. Então, $g \in \widetilde{y}+$ $I_{2}\left(M_{F}\right)$, onde $\tilde{g}(x, y, 0)=0$ para todo $(x, y) \in\left(\mathbb{C}^{2}, 0\right)$ e, portanto, $g \in \pi^{*}\left(\mathcal{M}_{\mathbb{C}, 0}\right) \mathcal{O}_{3} / I_{2}\left(M_{F}\right)$. Reciprocamente, dado $\bar{g} \in \pi^{*}\left(\mathcal{M}_{\mathrm{C}, 0}\right) \mathcal{O}_{3} / I_{2}\left(M_{F}\right)$, temos $\bar{g}=\sum_{i} \pi^{*}\left(h_{i}\right) \bar{g}_{i}$ com $h_{i} \in \mathcal{M}_{\mathbb{C}, 0} \mathrm{e}$ $\bar{g}_{i} \in \mathcal{O}_{3} / I_{2}\left(M_{F^{*}}\right)$. Como $i^{*}\left(\pi^{*}\left(h_{i}\right)\right)=0$ segue que $i^{*}(\bar{g}) \cdots 0$, isto $\dot{a}, \bar{g} \in \mathrm{ker} i^{\times}$. Conchúmos entăo que ker $i^{*}=\pi^{*}\left(\mathcal{M}_{2,0}\right) \mathcal{O}_{3} / I_{2}\left(M_{*}\right)$. Assim,

$$
\frac{\mathcal{O}_{X, 0}}{\pi^{\times}\left(\mathcal{M}_{, 0}\right) \mathcal{O}_{X, 0}} \simeq \frac{\mathcal{O}_{2}}{I_{2}\left(M_{f}\right)}
$$

c, portanto,

$$
\operatorname{dim}_{\mathbb{0}} \frac{\mathcal{O}_{X, 0}}{\pi^{*}\left(\mathcal{M}_{C, 0}\right) O_{X, 0}}=\operatorname{dim}_{\mathbb{C}} \frac{\mathcal{O}_{2}}{I_{2}\left(M_{f}\right)}
$$

So $u \neq 0$

$$
\pi^{-1}(u)=\left\{(x, y, u) /(x, y) \in \Sigma_{f u}^{1,1}\right\}
$$

onde $f_{u}$ ć um germe estável e a cardinalidade de $\pi^{-1}(u)=\operatorname{dim}_{\mathbb{C}} \mathcal{O}_{2} / I_{2}\left(M_{f}\right) . \operatorname{Mas}^{-1}(u)$ corresponde aos pontos de cúspide de $f_{u}$. Portanto, as pré-imagens são todas simples. De 
fato, se $f(x, y)=\left(x, y^{3}+x y\right)$ entào

$$
M_{f}=\left(\begin{array}{cc}
1 & 0 \\
y & 3 y^{2}+x \\
1 & 6 y
\end{array}\right)
$$

0

$$
\operatorname{dim}_{L} \frac{\mathcal{O}_{2}}{I_{2}\left(M_{f}\right)}=\operatorname{dim}_{\mathbb{C}} \frac{\mathcal{O}_{2}}{\left\langle 3 y^{2}-x, 6 y^{2}-3 y^{2}-x, 6 y\right\rangle}-\operatorname{dim}_{\Upsilon} \frac{\mathcal{O}_{2}}{\langle x, y\rangle}-1
$$

Purtanto $f_{u}$ tem dinn $\frac{O_{2}}{I_{2}\left(M_{f}\right)}$ cúspides.

O valor de d(f) pode agora ser calculado usando (4.4) e a fórmula obtida na proposição acima. Segne da fómula de Milnor e de (4.4) que

$$
(f)+d(f)=\frac{\mu(\Delta(f))-\mu(S(f))}{2}
$$

Lema 4.2.8 Sejam $f \in \mathcal{M}_{2} \mathcal{O}_{2.2}$ e

$$
M_{j}=\left(\begin{array}{ll}
p_{x} & p_{y} \\
q_{x} & q_{y} \\
J_{x} & J_{y}
\end{array}\right)
$$

onde $p$ eq säo us componentes de $f, J$ é o detemmante jacobiano de $f$ e os subscritos indicam derivadas parciais. Seja $I_{2}\left(M_{f}\right)$ o ideal gerado pelos menores de ordem 2 da matriz $M_{f}$. Enläo a sequência

$$
0 \longrightarrow \frac{\mathcal{O}_{2}}{\left\langle p_{x}, p_{y y}\right\rangle} \stackrel{q_{x} J_{y,} g_{y} J_{x:}}{\longrightarrow} \frac{\mathcal{O}_{2}}{\left\langle J_{1} p_{x} J_{y}-p_{y} J_{x}\right\rangle} \longrightarrow \frac{\mathcal{O}_{2}}{I_{2}\left(M_{f}\right)} \longrightarrow 0
$$

exata.

Demonstração A sequência é exata em $\frac{\mathcal{O}_{2}}{I_{2}\left(M_{f}\right)}$, pois $\phi$ é a projeção canônica e, portanto sobrejetiva.

Consideremos a sequência

$$
0 \cdots>\mathcal{O}_{2}^{2} \stackrel{M_{f}}{\longrightarrow} \mathcal{O}_{2}^{3} \stackrel{\Delta}{\rightarrow} \mathcal{O}_{2} \quad>\frac{\mathcal{O}_{2}}{I_{2}\left(M_{f}\right)} \quad \rightarrow 0
$$

onde $\Delta: \mathcal{O}_{2}^{3}-, \mathcal{O}_{2}$ é dada por

$$
\Delta\left(f_{1}, f_{2}, f_{3}\right)=f_{1}\left(q_{x}, J_{y}-q_{y} J_{x}\right)-f_{2}\left(p_{x}, J_{y}-p_{y}, J_{x}\right)+f_{3} J
$$


Como $f$ é finitamente determinada, dime $\frac{\mathcal{O}_{2}}{I_{2}\left(M_{f}\right)}=c(f)<\infty$. Segue do Corolário 2.4 .2 que (4.9) é exata em $\mathcal{O}_{2}^{3}$. Togo, a aplicação $q_{x} J_{y}-q_{y} J_{x}$ é injetiva. De fato, seja $g \in \mathcal{O}_{2}$ lal que $g\left(q_{x} J_{y}-q_{y} J_{x}\right) \in\left\langle J_{:} p_{x} J_{y}-p_{y} J_{x}\right\rangle$. Fntão existem $\alpha$ e $\beta \in \mathcal{O}_{2}$ tais que

$$
g\left(q_{x} J_{y} \cdot q_{y} J_{x}\right)+\alpha\left(p_{x} J_{y}-p_{y} J_{x}\right)+\beta J \quad 0
$$

ou scjal, $\Delta(y, \quad \alpha, \beta)=0 . \operatorname{Logo}(g,-\alpha, \beta) \in \operatorname{ker} \Delta=\operatorname{Im} M_{f}$. Segue que existem $\gamma^{\prime}$ ? $\eta \in \mathcal{O}_{2}$ tais que

$$
(g, \alpha, \beta)=M_{f}(\gamma, \eta)=\eta\left(p_{x}, q_{x}, J_{x}\right)+\eta\left(p_{y}, q_{y}, J_{y}\right)
$$

oll seja, $g=\gamma p_{x}+\eta p_{y} \in\left\langle p_{x}, p_{y}\right\rangle$. Portanto, (4.8) é exata em $\mathcal{O}_{2} /\left\langle p_{x}, p_{y}\right\rangle$.

Basta mostrar agora que (1.8) o exata em $\mathcal{O}_{2} /\left\langle J, p_{x} J_{y}-p_{y} J_{x}\right\rangle$. Temos que

$$
\operatorname{ker} \phi-\frac{I_{2}\left(M_{f}\right)}{\left\langle J_{,} p_{x} J_{y}-p_{y}, J_{x}\right\rangle}=\left(q_{x} J_{y}-q_{y} J_{x}\right)\left(\left\langle J_{,} p_{x} J_{y} \sim p_{y}, J_{x}\right\rangle\right)
$$

$\log \operatorname{ker} \phi \subset \operatorname{In}\left(q_{x} J_{y}-q_{y} J_{x}\right)$. Reciprocamente, se $\bar{g} \in \operatorname{Im}\left(q_{x} J_{y}-q_{y} J_{d x}\right)$ entào $\bar{g}$ o da forma

$$
h\left(q_{x} J_{y}-q_{y} J_{x}\right)+\left\langle J_{i} p_{x} J_{y}-p_{y} J_{x}\right\rangle
$$

para algum $h \in \mathcal{O}_{2}$. Logo,

$$
\phi(\bar{g})=h\left(q_{y} J_{y} \cdots q_{y} J_{x}\right)+I_{2}\left(M_{j}\right) \in I_{2}\left(M_{j}\right)
$$

ou seja, $\bar{q} \in \operatorname{her} \phi$. Portanto 1.8 é exata em $\mathcal{O}_{2} /\left\langle J, p_{x} J_{y}-p_{y} J_{x}\right\rangle$.

Corolário 4.2.9 Seja $f \in \mathcal{M}_{2} \mathrm{O}_{2,2}$ um germe finitamenté determinado. Entäo

$$
c(f)=\mu(S(f))+m(f)-2
$$

onde $m(f)=\operatorname{dim}_{0} \frac{\mathrm{O}_{2}}{\left\langle f_{1}, f_{2}\right\rangle}$ é grau da aplicacào $f=\left(f_{1}, f_{2}\right)$.

Demonstraçāo Mostremos primeiramente que podernos escolher coordenadas de modo que $p^{-1}(0) \cap S(f)-\{0\}$. Temos que $f$ ó $k$-determinada para algum $k$. Entäo, para todo $g \in \mathcal{M}_{2} \mathcal{O}_{2,2}$ tal que $j^{k} g(0)=j^{k} f(0)$ temos que $f$ o $\mathcal{A}$-equivalente a $g$. Portanto $f$ é $\mathcal{K}$-equivalente a $g$. Segue daí que $f$ é $\mathcal{K}$-determinada. Pela Proposição 1.5 .3 temos entäo que $f$ é $\mathcal{C}$-determinala. P'or $[29$, pg 193], $m(f)<\infty$. Segue do 'Teorema 3.2.j que $f^{1}(0)$ tem um número finito de pontos e, portanto, $f^{-1}(0) \Gamma S(f)$ tem um número finito de pontos. Observemos que $S(f) \cdot p^{-1}(0)$ e $q^{-1}(0)$ são curvas em $\mathbb{C}^{2}$. Se $p^{-1}(0)$ 
coincide com $S(f)$ numa vizinhança da origem, então $q^{1}(0)$ não pode coincidir com $S(f)$ nesta vizinhança, pois $f^{1}(0) \cap S(f)$ tem um número finito de pontos. A menos de una mudança de coordenadas podemos entào assumir que $p^{-1}(0) \cap S(f)=\{0\}$.

Consideremos o ideal $I \quad\langle J, p\rangle \subset \mathcal{O}_{2}$, onde $J(x, y)$ é o determinante jacoliano de $f$ em $(x, y)$. A variedade analítica definida por $I$ ó dada por

$$
\left\{(x, y) \in \mathbb{C}^{2} / p(x, y)=0 \text { e } J(x, y)=0\right\}=\left\{(x, y) \in p^{1}(0) \cap S(f)\right\}=\{0\}
$$

Portanto, I define um conjunto analítico de codimensão 2 e tem dois geradores. Como 0 ć singularidade isolada, cntão $I$ define um ICIS. O ideal $\langle J\rangle$ tem 1 gerador e define a curva $S(f)$, que tem dimensão $1 . \log$ $\langle J\rangle$ também define um ICIS.

Segue de $[16,5.11$ (a) $\mid$ que

$$
\mu(J, p)+\mu(J)=\operatorname{dim} \frac{\mathcal{O}_{2}}{\left\langle J \cdot p_{x} J_{y}-p_{y} J_{x}\right\rangle}
$$

onde $\mu$ o o mumero de Milnor do ICIS definido por $I$. Votemes que $\mu(J)=\mu(S(f))$. Como o ICIS definido por $I$ ó 0-dimensional, segue de $|16,5.12|$ que

$$
\mu(J, p)=\operatorname{dini}: \frac{\mathcal{O}_{2}}{\langle J, p\rangle}-1
$$

Assim

$$
\operatorname{dim}_{\mathbb{C}} \frac{\mathcal{O}_{2}}{\left\langle J, p_{x} J_{y}-p_{y} J_{x}\right\rangle}=\mu(S(f))+\operatorname{dim} \frac{\mathcal{O}_{2}}{\left\langle J_{,} p\right\rangle}-1
$$

Vimos que o conjunto $f^{-1}(0)$ possui um número finito de pontos. Logo podemos tomar $f^{-1}(0)=\{0\}$, on seja, $p^{-1}(0) \cap q^{-1}(0)=\{0\}$. Assim, $\langle p, q\rangle$ tem dois geradores e define um conjunto de codimensão 2. Segue daí que $\langle p, q\rangle$ define um ICIS. O ideal $\langle p\rangle$ tem 1 gerador e define uma variedade de codimensão 1. Portanto também define um ICIS. Aplicando $[16,5.11$ (a)] obtemos

$$
\operatorname{dim}_{\mathbb{C}} \frac{\mathcal{O}_{2}}{\left\langle J_{, p\rangle}\right\rangle}=\mu(p)+\mu(p, q)
$$

e por $|16, \check{\jmath} .12|$

$$
\mu(p, q)-\operatorname{dim} \frac{\mathcal{O}_{2}}{\langle p, q\rangle}=m(f)-1
$$

Logo

$$
\lim _{\mathfrak{r}} \frac{\mathcal{O}_{2}}{\langle J, p\rangle}=\mu(p)+n(f)-1
$$

Portanto

$$
\operatorname{dim}_{\mathbb{C}} \frac{\mathcal{O}_{2}}{\left\langle J_{,} p_{x} J_{y}-p_{y} J_{x}\right\rangle}=\mu(p)+\mu(S(f))+m(f)-2
$$


Segue do Lema 4.2 .8 que

$$
\begin{gathered}
\operatorname{dim} \operatorname{Im}\left(q_{x} J_{y} \quad q_{y} J_{x:}\right)=\operatorname{dim} \operatorname{ker} \phi \\
\operatorname{dim} \operatorname{Im}\left(q_{x} J_{y}-q_{y} J_{x}\right) \cdot \operatorname{dim} \frac{\mathcal{O}_{2}}{\left\langle p_{x}, p_{y y}\right\rangle} \\
\operatorname{dim} \operatorname{Im} \phi-\operatorname{dim} \frac{\mathcal{O}_{2}}{I_{2}\left(M_{f}\right)}
\end{gathered}
$$

ou seja,

$$
\operatorname{dim}_{\mathbb{C}} \frac{\mathcal{O}_{2}}{\left\langle p_{x} \cdot p_{y}\right\rangle}-\operatorname{dim}_{\odot} \frac{\mathcal{O}_{2}}{\left\langle J_{, p_{x}} J_{y}-p_{y}, J_{x}\right\rangle}-c(f)
$$

Logo

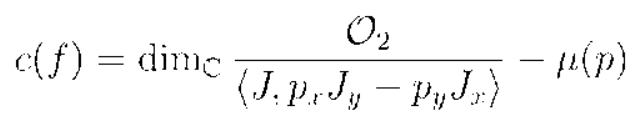

Substituindo a expressão acima cm (4.10) obtemos o resultado.

A seguir mostramos a invariancia analítica de of $f)$ o $d(f)$, ou seja, se dois germes $f$ e g săo $\mathcal{A}$-equivalentes, então $r(f)=r(g)$ e $d(f)=d(g)$. Trata-se de um caso particular do Teorema 1.2.11.

Proposição 4.2.10 Seja $f \in \mathcal{M}_{2} \mathcal{O}_{2,2}$ um germe finitamente determinado. Os números $c(f)$ ed(f) săo invarianles analíticos.

Demonstração Seja $g \in \mathcal{M}_{2} \mathcal{O}_{2,2}$ tal que $f \sim_{4} g$, ou seja, existem germes de difeomorfismos $\phi . \psi^{\prime}:\left(\mathbb{C}^{2}, 0\right) \rightarrow\left(\mathbb{C}^{2}, 0\right)$ tais que $f=\psi \circ g \circ \phi$. Temos que

$$
\left.\left.\operatorname{det} d_{x} f\right]=\operatorname{det}_{i} d_{g \circ \phi(x)} \psi^{i}\right] \operatorname{det}\left[d_{\phi(x)} g\right] \operatorname{det}\left[d_{x} \dot{\phi}\right.
$$

Como $d_{g \cup \varphi(x)} \dot{\psi}$ e $d_{x} \phi$ são isomorlismos, entào det $\left.d_{\phi \phi(x)} g\right]-0$ se, e somente se, $\operatorname{det}\left[d_{x} f\right]=0$. Segle que

$$
\left.\phi(S(f))=\left\{\phi(x) \in\left(\mathbb{C}^{2}, 0\right) / \operatorname{det}\left[d_{\phi(x)} g\right]=0\right\}=\left\{y \in\left(\mathbb{C}^{2}, 0\right) / \operatorname{det} d_{y} g\right]=0\right\}=S(g)
$$

Ainda,

$$
\psi(\Delta(g))=\psi(g(S(g)))=\psi \circ g \circ \phi(S(f))=f(S(f))=\Delta(f)
$$

Como o número de Milnor é um invariante analítico então

$$
\mu(S(f))=\mu(S(g))
$$


c

$$
\mu(\Delta(f)) \cdots \mu(\Delta(g))
$$

Mostremos agora que $m(f)=m(g)$. Para isto, basta mostrar que $I_{f}=\left\langle f_{1}, f_{2}\right\rangle$ o $I_{g}\left\langle g_{1}, g_{2}\right\rangle$ säo isomorfos, onde $f_{1} \cdot f_{2} e g_{1} \cdot g_{2}$ sào as funçoos coordenadas respectivanente de $f$ eg. De fato, se $I_{f} \simeq I_{g}$ temos

$$
m(f)=\operatorname{dim}_{C} \frac{\mathcal{O}_{2}}{l_{f}}-\lim _{\mathbb{C}} \frac{\mathcal{O}_{2}}{I_{g}} \ldots m(g)
$$

Mostremos primeiramente no caso $\mathrm{cm}$ que $\phi=I d \mathrm{c}^{n}$. Se $\psi_{1}, \psi_{2}$ são as funções coordenadas de $\psi$ então

$$
f-\left(f_{1}, f_{2}\right)=\left(\psi_{1} \circ g, \psi_{2} \circ g\right)
$$

Como $\psi_{i}(0,0)=0, i=1,2$, segue do Lemia 1.4.1 que

$$
\psi_{i}(x, y)=\alpha_{1 i}(x, y) x+\alpha_{2 i}(x, y) y
$$

onde $\alpha_{j i} \in \mathcal{O}_{22}, i, j-1,2 . \operatorname{Logo}$

$$
f_{i}(x, y)=\psi_{i}(g(x, y))=\alpha_{1 i}(y(x, y)) g_{1}(x, y)+\alpha_{2_{i}}(x, y) g_{2}(x, y)
$$

ou seja, $f_{i} \in I_{g}, i=1,2$. Portanto $I_{f} \subseteq I_{g}$. Por sinctria, $I_{f}=I_{g}$. Consideremos agora $\phi$ um difeomorfismo qualduer. Segue do caso anterior que $I_{f}=I_{g c a j}$. Consideremos o isomorfismo induzido por $\theta$

$$
\begin{aligned}
\phi^{*}: \mathcal{O}_{2} & \longrightarrow \mathcal{O}_{2} \\
k & \longmapsto k \circ f
\end{aligned}
$$

Temos que $\phi^{*}\left(I_{g}\right)=I_{g \circ \phi}$. De fato, seja $\ddot{g} \in I_{g}$. Entĩo

$$
\widetilde{g}(x, y)=\beta_{1}(x, y) g_{1}(x, y)+\beta_{2}(x, y) g_{2}(x, y)
$$

onde $\beta_{1}, \beta_{2} \in \mathcal{O}_{2} \mathrm{e}$

$$
\begin{gathered}
\phi^{*}(\widetilde{g}(x, y)) \cdots \phi^{*}\left(\beta_{1}(x, y)\right) \phi^{*}\left(g_{1}(x, y)\right)+\phi^{*}\left(\beta_{2}(x, y)\right) \phi^{*}\left(g_{2}(x, y)\right) \\
-\left(\beta_{1} \circ \phi\right)(x, y)\left(g_{1} \circ \phi\right)(x, y)-\left(\beta_{2} \circ \phi\right)(x, y)\left(g_{2} \circ \phi\right)(x, y)
\end{gathered}
$$

ou seja, $\phi^{*} \circ \tilde{g} \in I_{g \circ \phi}$. $\operatorname{Logo} \phi^{*}\left(I_{g}\right) \subseteq I_{g \circ \phi}$. Reciprocimente, seja $\tilde{g} \in I_{g \circ \dot{\varphi}}$. Então

$$
\dddot{y}=\gamma_{1} g_{1} \circ o+\gamma_{2} g_{2} \circ \phi
$$


onde $\gamma_{1}, \gamma_{2} \in \mathcal{O}_{2}$. Como $\phi^{*}$ é isomorfismo, existem $\beta_{1}, \beta_{2} \in \mathcal{O}_{2}$ tais que $\phi^{*}\left(\beta_{1}\right)=\gamma_{1}$ e $\phi^{*}\left(\beta_{2}\right)=\gamma_{2} . \log 0$

$$
\widetilde{g}=\phi^{*}\left(\beta_{1}\right) \phi^{*}\left(g_{1}\right)+\phi^{*}\left(\beta_{2}\right) \wp^{*}\left(g_{2}\right)=\phi^{*}\left(\beta_{1} g_{1}+\beta_{2} g_{2}\right)
$$

on seja, $\ddot{g} \in \hat{q}^{*}\left(I_{g}\right)$. Portanto $I_{f}-I_{g o c} \simeq I_{i g}$.

Segue do Corolário 4.2 .9 que

$$
c(f)=\mu(S(f))+m(f)-2=\mu(S(g))+m(g)-2=r(g)
$$

(?)

$$
d(f)=\frac{1}{2}(\mu(\Delta(f))-\mu(S(f)))-c(f) \quad \frac{1}{2}(\mu(\Delta(g))-\mu(S(g)))-c(g)=d(g)
$$

Teorema 4.2.11 Seja $f \in \mathcal{M}_{2} \mathrm{O}_{2,2}$ um geme finitamente determinado. Os números (f) ed(f) säo invariantes topológicos.

Demonstraçāo Sejal $g \in \mathcal{M}_{2} \mathcal{O}_{2,2}$ tal que existem germess de homeomorfismos $\phi, \psi\left(\mathbb{C}^{2}, 0\right) \rightarrow\left(\mathbb{C}^{2}, 0\right)$, onde $f=0 g \circ 0$. Pela Proposição 4.2 .1 temos gue $S(f)$ c $S(g)$ são curvas. Segue do leorema 1.3 .3 que existe una vizinhança $U$ da origem tal que, se $y \in U \cap S(f)$, o germe de $f$ em $y$ é estável. Portanto, o germe de $f$ em $y$ é uma dobra ou una cúspide de Whitney. $\Lambda$ cúspide de Whitney corresponde a uma cúspide em $S(f)$. Como cúspides ocorrem isoladamente, podemos encolher $U$ de forma que o germe de $f$ em y seja uma dobra. O mesmo ocorre para $g$. Como $\phi$ é homeomorfismo, $\phi$ leva dobra em dobra. $\log \phi(S(f))=S(g)$. Analoganente $\psi(\Delta(g)) \quad . \Delta(f)$. Ainda, segue do $|28|$ rue o múmero de Milnor de uma hipersuperfície singular é um invariante topológico, logo

$$
\mu(S(f))=\mu(S(g)) \quad \mu(\Delta(f))=\mu(\Delta(g))
$$

Notenos que $\mathbb{C}$ e um anel de Cohen- Nacaulay, pois dim $\mathbb{C}=-\operatorname{depth} \mathbb{C}=0$. Pelo Teorema 3.2.j temos cntão que $m(f)$ é igual à cardinalidade de $f^{-1}\left(v_{0}\right)$ para $v_{0}$ suficientemente próximo da origem. $\log \%, m(f)=m(g)$.

O resultado segue do Corolário 4.2 .9 e de (1.7).

Corolário 4.2.12 Seja $F:\left(\mathbb{C}^{2} \times \mathbb{C}, 0\right) \rightarrow\left(\mathbb{C}^{2} \times \mathbb{C}, 0\right), F(x, t)=\left(f_{l}(x), t\right)$, um desdobramento de um germe de aplicaça a holomorfa finitamente determinado $f=f_{0}$. Suponhamos que $f_{t}$ é instável em 0 para todo t. Endăo $F$ é um desdobramento topologicamente trivial se, e somente se, $c\left(f_{l}\right)$ e $d\left(f_{t}\right)$ säo constantes. 
Demonstração Se $F$ é um desdobramento topologicamente trivial, então todas as $f_{t}$ săo topologicamente equivalentes. Segue de Corolário 4.2 .11 que $c\left(f_{t}\right)$ e $d\left(f_{t}\right)$ săo constinintes.

Reciprocamente, suponhanos que $\left(f_{t}\right)$ e $d\left(f_{i}\right)$ sejam constantes. Como $d\left(f_{t}\right)$ é constante, segue do Corolário 4.2 .9 que $\mu\left(S\left(f_{l}\right)\right)$ é constante. Por $(4.7), c\left(f_{t}\right)$ e $d\left(f_{l}\right)$ constantes implicam (que $\mu\left(\Delta\left(f_{1}\right)\right)$ é constante. () resultado segue de $[6,9.9$.

\subsection{Germes de corank 1}

Nesta seção obtemos uma fórmula que simplifica o cálculo de $c(f)$ a $d(f)$ quando $f$ tem corank 1. A idéia central é considerar $f$ como um desdobramento a urn parâmetro de um germe de função do tipo $A_{k}$ e. conno tal, induzida do desdobranento versal de $A_{k}$.

Nesta segão fo um germe em $\mathcal{M}_{2} \mathcal{O}_{2: 2}$ de corank 1. No resultado seguinte obtemos uma pré forma normal para tais germes.

Teorema 4.3.1 Seja $f=\left(f_{1}, f_{2}\right):\left(\mathbb{C}^{2} .0\right) \rightarrow\left(\mathbb{C}^{2}, 0\right)$ um germe finitamente determinado de corank 1. Lintâo f é $\mathcal{A}$-equivalente a um germe da forma

$$
\left(x, y^{n}+\sum_{i=1}^{n-2} a_{i}(x) y^{i}\right)
$$

onde $n-\operatorname{dim} \frac{\mathcal{O}_{2}}{\left\langle f_{1}, f_{2}\right\rangle}$ é o grau de $f$.

Demonstração Como $f$ tem corank 1, existe uma submatriz de ordem 1 da matrì de $d_{0} f$ cujo determinante é não nuko. Fazendo mudancass de coordenadas na fonte e na meta se necessário, podemos supor que $\frac{\partial f_{1}}{\partial x}(0) \neq 0$. Consideremos então o germe $\phi:\left(\mathbb{C}^{2}, 0\right) \rightarrow\left(\mathbb{C}^{2}, 0\right)$, dado por $\phi(x, y)=\left(f_{1}(x, y), y\right)$. A matriz de $d_{0} \phi \dot{e}$

$$
\left(\begin{array}{cc}
\frac{\partial f_{1}}{\partial x}(0) & \frac{\partial f_{1}}{\partial y}(0) \\
0 & 1
\end{array}\right)
$$

Logo, $d_{0} \phi \dot{e}$ isomorfismo e, portanto, $\phi$ é germe de difeomortismo. Podemos então considerar o germe $\phi \quad\left(\varphi_{1}(x, y), \varphi_{2}(x, y)\right)$. Temos:

$$
(x, y)=\phi \circ \phi^{-1}(x, y)=\left(f_{1}\left(\phi^{-1}(x, y)\right), \phi_{2}(x, y)\right)
$$

Portanto

$$
f \circ \phi^{-1}(x, y)=f\left(\phi^{-1}(x, y)\right)=\left(f_{1}\left(\phi^{-1}(x, y)\right), f_{2}\left(\phi^{-1}(x, y)\right)\right)=\left(x, f_{2}\left(\phi^{-1}(x, y)\right)\right)
$$


Segue que. a menos de mudança de coordenadas na fonte, $f \circ$ da forma $f(x, y)=$ $\left(x, \int_{2}(x, y)\right)$.

Como $\int$ ó finitamente determinada temos que $f_{2}(0, y) \neq 0$. Assim, $f_{2}(0, y)=y^{l} g(y)$ com $g(0) \neq 0$. Como

$$
n-\operatorname{dim}_{-} \frac{\mathcal{O}_{2}}{\left\langle\alpha, f_{2}(0, y)\right\rangle}
$$

segue quel $=n$.

Como conseguência do Teorema 1.4.4 podemos escrever

$$
f(x, y) \cdot\left(x,\left(\alpha_{0}(x)-\cdots+\alpha_{n} 1(x) y^{n} 1+y^{n}\right) u\right)
$$

onde $u \in \mathcal{O}_{2}$ e um elemento invertível.

Consideremos o seguinte germe de difeomorfismo

$$
\begin{aligned}
H:\left(\mathbb{C}^{2}, 0\right) & \longrightarrow\left(\mathbb{C}^{2}, 0\right) \\
(X, Y) & \longmapsto\left(X, u^{-1} Y \cdots \alpha_{0}(X)\right)
\end{aligned}
$$

Assim, $\varphi \circ f(x, y)=\left(x, \alpha_{1}(x) y-\cdots+\alpha_{n-1}(x) y^{n}{ }^{1}+y^{n}\right)$.

Considerando agora o germe de difeomorfismo

$$
\begin{aligned}
\varphi:\left(\mathbb{C}^{2}, 0\right) & \longrightarrow\left(\mathbb{C}^{2}, 0\right) \\
(x, y) & \longrightarrow\left(x, y-\frac{\alpha_{n-1}(x)}{n}\right)
\end{aligned}
$$

temos

$$
\begin{gathered}
f \circ \varphi(x, y)=f\left(x, y \quad \begin{array}{c}
\alpha_{n-1}(x) \\
n
\end{array}\right)=\left(x, \alpha_{1}(x)\left(y-\frac{\alpha_{n-1}(x)}{n}\right)+\alpha_{2}(x)\left(y-\frac{\alpha_{n} 1(x)}{n}\right)^{2}+\cdots\right. \\
\left.+\alpha_{n-1}(x)\left(y-\frac{\alpha_{n-1}(x)}{n}\right)^{n-1}+\left(y-\frac{\alpha_{n-1}(x)}{n}\right)^{n}\right)=\left(x, a_{0}(x)+a_{n-2}(x) y+\cdots+a_{1}(x) y^{n}{ }^{2}+y^{n}\right)
\end{gathered}
$$

Finalmente, após mudança de coordemadas na meta $\int$ é $\mathcal{A}$-equivalente a

$$
(x, y))_{1} \quad,\left(x, a_{n-2}(x) y-\cdots+a_{1}(x) y^{\prime \prime 2}+y^{n}\right)
$$

Se $m(f)=k+1$, segue do teorema acima que, a menos de mudança de coordenadas na fonte e na meta podenos escrever

$$
f(x, y) \cdots\left(x, y^{k+1}+a_{1}(x) y^{k-1}+\cdots+a_{k-1}(x) y\right)
$$

Os coeficientes $u_{i}(x)$ do $y^{k-i}$ determinam a aplicação 


$$
\begin{aligned}
a_{j}: \mathbb{C} & \longrightarrow \mathbb{C}^{k-1} \\
x & \vdots \cdots, \quad\left(a_{1}(x), \ldots, a_{k-1}(x)\right)
\end{aligned}
$$

Podemos ver $f$ como um desdobramento a 1 parámetro de uma singularidade do tipo $A_{k}$

$$
\begin{aligned}
f:(\mathbb{C} \times \mathbb{C}, 0) & \longrightarrow(\mathbb{C} \times \mathbb{C}, 0) \\
(x, y) & \longmapsto(x, y(x, y))
\end{aligned}
$$

onde $g(x, y)=y^{k+1}+a_{1}(x) y^{k-1}+\cdots+a_{k-1}(x) y e g(x, 0)=y^{k-1}$

A aplicação $a_{f}=\left(a_{1}, \ldots, a_{k} 1\right)$ pode ser vista como uma aplicação da base do dessdobramento $f$ na base do desdobramento versal $F$ de $A_{k}$ :

$$
\begin{aligned}
F^{\prime}:\left(\mathbb{C}^{k-1} \times \mathbb{C}, 0\right) & \longrightarrow\left(\mathbb{C}^{k-1} \times \mathbb{C}^{\mathfrak{n}}, 0\right) \\
\left(u_{1}, \ldots, u_{k-1}, y\right) & \longmapsto\left(u_{1}, \ldots, u_{k-1}, y^{k+1}+u_{1} y^{k-1}+\cdots+u_{k-1} y\right)=\left(u, g_{u}(y)\right)
\end{aligned}
$$

Definimos agora os conjuntos de bifurcação $B, B_{C}$ e $B_{D}$ na base do desdobramento $F$ :

$$
\begin{gathered}
B_{C^{\prime}}=\left\{u \in \mathbb{C}^{k-1} / g_{u} \text { tem um ponto crítico degenerado }\right\} \\
B_{b)}=\left\{u \in \mathbb{C}^{k-1} / g_{u} \text { tem dois pontos críticos com o mesmo valor crítico }\right\} \\
B=\left\{u \in \mathbb{C}^{k-1} / g_{u} \text { nào é estável }\right\}
\end{gathered}
$$

Segue de [11, Proposição 2.2] que $B \ldots B_{C} \cup B_{D}$.

Sejam $\Delta_{\text {sing }}$ o conjunto dos pontos singulares de $\Delta\left(F^{\prime}\right)$ e $\pi: \mathbb{C}^{k} 1 \times \mathbb{C} \rightarrow \mathbb{C}^{k-1}$ a projeção na base do desdobramento $F$. Segue de $[23, \$ 6]$ que

$$
\begin{gathered}
B_{C}=\left\{u \in\left(\mathbb{C}^{k-1}, 0\right) / \exists y \in(\mathbb{C}, 0) \text { tal que } \frac{\partial g_{u}}{\partial y}(y)=0 e \frac{\partial^{2} g_{u}}{\partial y^{2}}(y)=0\right\} \\
B=\pi\left(\Delta_{\text {sing }}\right)
\end{gathered}
$$

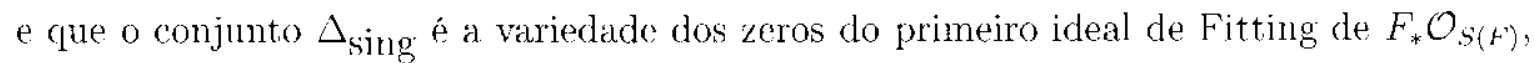
isto é, $\Delta_{\text {sing }}=V\left(\mathcal{F}_{1}\left(F_{*} \mathcal{O}_{S\left(K^{\prime}\right)}\right)\right)$. Dessa forma calculamos $B$. O conjunto $B_{D}$ pode então ser calculado dividindo a equação de $B$ pela de $B_{C}$.

Exemplos 4.3.2 1. Consideremos $f(x, y)=\left(x, y^{4}+a_{1}(x) y^{2}+a_{2}(x) y\right)$. Entäo

$$
F\left(u_{1}, u_{2}, y\right)-\left(u_{1}, u_{2}, y^{4}+u_{1} y^{2}+u_{2}, y\right)=\left(u, g_{u}(y)\right)
$$


Temos

$$
\begin{aligned}
S(F)= & \left.\left\{(u, y) \in\left(\mathbb{C}^{3}: 0\right) / d u_{1} \mid d_{(u, y)} F\right]=0\right\} \\
& \left\{(u, y) \in\left(\mathbb{C}^{3}: 0\right) / 4 y^{3}+2 u_{1} y+u_{2}=0\right\} \\
= & \left\{(u, y) \in\left(\mathbb{C}^{3}, 0\right) / u_{2}=-4 y^{3}-2 u_{1} y\right\} \\
\Delta(F)= & \left\{F\left(u_{1}, u_{2}, y\right) /\left(u_{1}, u_{2}, y\right) \in S(F)\right\} \\
= & \left\{\left(u_{1},-4 y^{3}-2 u_{1} y-u_{1} y,-3 y^{4}-u_{1} y^{2}\right)\right\}
\end{aligned}
$$

Temos que

$$
B_{c}:=\left\{u \in\left(\mathbb{C}^{2}, 0\right) / \exists y \subset(\mathbb{C}, 0) \text { tal que } 4 y^{3}+2 u_{1} y+u_{2}=0 \Leftrightarrow 12 y \cdot 2 u_{1}=0\right\}
$$

ou seja, $B_{C}$ e o discriminante do $4 y^{3}: 2 u_{1} y+u_{2}$. Com o anxílio do programa Maple calculamos este discriminante e, portanto, a equação de $B_{C}$ :

$$
8 u_{1}^{3}+27 u_{2}^{2}=0
$$

Queremes agora encontrar a equaçăo de B. Para isso, é necessário calcular primeiramente o primeiro ideal de Fitting de $F_{*} \mathcal{O}_{S(F)}$. Temos

$$
\begin{aligned}
\left.F\right|_{S\left(w^{\prime}\right)}: S(F) & \longrightarrow\left(\mathbb{C}^{3}, 0\right) \\
\left(u_{1},-4 y^{3}-2 u_{1} y, y\right) & \longmapsto\left(u_{1},-4 y^{3}-2 u_{1} y,-3 y^{4}-u_{1} y^{2}\right)
\end{aligned}
$$

Logo

$$
\frac{F_{*} \mathcal{O}_{S(F)}}{\mathcal{M}_{3} F_{*} \mathcal{O}_{S\left(F^{2}\right)}} \simeq \frac{\mathcal{O}_{2}}{\left\langle u_{1},-1 y^{3}-2 u_{1} y,-3 y^{4}-u_{1} y^{2}\right\rangle} \simeq \mathbb{C}\left\{1, y, y^{2}\right\}
$$

Sejan $U-u_{1}, V=-4 y^{3}-2 u_{1} y$ e $V--3 y^{4}-u_{1} y^{2}$. Com o auxílio do programa Maple encontramos as relaçós milre $1, y$ e $y^{2}$

$$
\begin{gathered}
4 W \cdot 1-3 V \cdot y-2 U \cdot y^{2}=0 \\
\left(\frac{3}{4} V^{2}: \frac{8}{3} U W\right) \cdot 1+0 . y+\left(4 W-\frac{1}{3} U^{2}\right) \cdot y^{2}-0 \\
16 W^{2} \cdot 1-V U^{2} \cdot y+\left(-9 V^{2} \cdot 16 U W-2 U^{3}\right) \cdot y^{2}=0
\end{gathered}
$$

obtendo assim a matriz

$$
\Lambda=\left(\begin{array}{ccc}
4 W & -3 V & \cdots 2 U \\
\frac{3}{4} V^{2}+\frac{8}{3} U W & 0 & 4 W \cdots \frac{1}{3} U^{2} \\
16 W^{2} & -V U^{2} & -9 V^{2}-16 U W-2 U^{3}
\end{array}\right)
$$

$O$ primeiro ideal de Fitting de $F_{*} \mathcal{O}_{S(F)}$ é o ideal gerado pelos menores de or- 
dem 2 de $\Lambda$. Tambóm com o auxílio do Maple, calculamos a equação de $\Delta_{\text {sing }}$-$V\left(\mathcal{F}_{1}\left(F_{*} \mathcal{O}_{S(F)}\right)\right)$, donde segue a equacião de $B=\pi\left(\Delta_{\text {sing }}\right)$

$$
u_{2}\left(8 u_{1}^{3}+27 u_{2}^{2}\right)=0
$$

Portanto al equação de $B_{l}$ é

$$
u_{2}=0
$$

2. Considerenos agora $f(x, y)=\left(x, y^{5}+a_{1}(x) y^{3}+a_{2}(x) y^{2}+a_{3}(x) y\right)$.

$$
F\left(u_{1}, u_{2}, u_{3}, y\right)=\left(u_{1}, u_{2}, u_{3}, y^{\bar{y}}+u_{1} y^{3}+u_{2} y^{2}+u_{3} y\right)=\left(u, g_{u}(y)\right)
$$

Temos

$$
\begin{aligned}
S(F)= & \left\{(u, y) \in\left(\mathbb{C}^{4}, 0\right) / \operatorname{det}\left[d_{(u, y)} F\right] \quad 0\right\} \\
= & \left\{(u, y) \in\left(\mathbb{C}^{4}, 0\right) / 5 y^{1}+3 u_{1} y^{2}+2 u_{2} y+u_{3}=0\right\} \\
= & \left\{(u, y) \in\left(\mathbb{C}^{4}, 0\right) / u_{3}=-5 y^{1}-3 u_{1} y^{2}-2 u_{2} y\right\} \\
\Delta(F)= & \left\{F\left(u_{1}, u_{2}, u_{3,} y\right) /\left(u_{1}, u_{2}, u_{3}, y\right) \in S(F)\right\} \\
= & \left\{\left(u_{1}, u_{2},-5 y^{1}-3 u_{1} y^{2}-2 u_{2} y,-4 y^{5}-2 u_{1} y^{3}-u_{2} y^{2}\right)\right\}
\end{aligned}
$$

Temos que

$B_{C}=\left\{u \in\left(\mathbb{C}^{3}, 0\right) / \exists y \in(\mathbb{C}, 0)\right.$ tal $(1 u c) 5 y^{4}+3 u_{1} y^{2}-2 u_{2} y+u_{3}=0$ e $\left.20 y^{3}+6 u_{1} y+2 u_{2}=0\right\}$ ou seja, $B_{C}$ é o discriminante de $5 y^{1}$ । $3 u_{1} y^{2}+2 u_{2} y+u_{3}$. Com o auxílio do programa Mapple, emcontramos a equação de $B_{C}$

$$
-81 u_{1}^{4} u_{3}-360 u_{1}^{2} u_{3}^{2}-400 u_{3}^{3}+135 u_{2}^{1}+27 u_{1}^{3} u_{2}^{2}-5 \cdot 10 u_{1} u_{2}^{2} u_{3}-0
$$

Ainda, $\left.F\right|_{S(F)}: S(F) \rightarrow\left(\mathbb{C}^{4}, 0\right)$ o dada por

$$
\left.F\right|_{s\left(F_{1}\right)}\left(u_{1}, u_{2},-5 y^{1}-3 u_{1} y^{2}-2 u_{2} y, y\right)=\left(u_{1}, u_{2},-5 y^{4}-3 u_{1} y^{2}-2 u_{2} y,-4 y^{5}-2 u_{1} y^{3}-u_{2} y^{2}\right)
$$

$\log ()$

$\frac{F_{\times} \mathcal{O}_{S(F)}}{\mathcal{M}_{1} F_{\times} \mathcal{O}_{S(F)}} \simeq \frac{\mathcal{O}_{3}}{\left\langle u_{1}, u_{2}:-5 y^{1}\right.} \cdot \frac{u_{1} y^{2}-2 u_{2} y,-4 y^{5}}{\left.-2 u_{1} y^{3}-u_{2} y^{2}\right\rangle} \simeq \mathbb{C}\left\{1, y, y^{2}, y^{3}\right\}$

Sejam $U=u_{1}, V=-5 y^{4}-3 u_{1} y^{2}-2 u_{2} y e W=\cdots 4 y^{5}-2 u_{1} y^{3}-u_{2} y^{2}$. Com o auxílio 
do programa Maple encontramos as relações entre $1, y, y^{2}$ e $y^{3}$

$$
\begin{gathered}
-5 Z \cdot 1+4 W \cdot y+3 V \cdot y^{2}+2 U \cdot y^{3}=0 \\
\frac{2}{5} U W \cdot 1+\left(-5 Z-\frac{4}{5} U V\right) \cdot y+\left(4 W-\frac{6}{5} U^{2}\right) \cdot y^{2}+3 V \cdot y^{3}=0 \\
\left(-\frac{1}{2} Z U-\frac{3}{5} V W\right) \cdot 1-\frac{6}{5} V^{2} \cdot y+\left(5 Z-\frac{23}{10} U V\right) \cdot y^{2}+\left(4 W-U^{2}\right) \cdot y^{3}=0 \\
\left(4 W^{2}+\frac{55}{4} V Z-\frac{6}{5} U^{2} W\right) \cdot 1-\frac{12}{5} U^{2} V y+\left(14 U W-\frac{9}{4} V^{2} \quad \frac{18}{5} U^{3}\right) \cdot y^{2}+\left(25 Z+\frac{15}{2} U V\right) \cdot y^{3}=0
\end{gathered}
$$

obtenclo assim a matri\%

$$
\Lambda=\left(\begin{array}{cccc}
-5 Z & 4 W & 3 V & 2 U \\
-\frac{2}{5} U W & -5 Z-\frac{1}{5} U V & 4 W-\frac{6}{5} U^{2} & 3 V \\
\frac{1}{2} Z U-\frac{3}{5} V W & -\frac{6}{5} V^{2} & -5 Z-\frac{23}{10} U V & 4 W-U^{2} \\
4 W^{2}+\frac{55}{4} V Z-\frac{6}{5} U^{2} W & -\frac{12}{5} U^{2} V & 14 U W \cdot \frac{9}{4} V^{2}-\frac{18}{5} U^{3} & 25 Z+\frac{15}{2} U V
\end{array}\right)
$$

O primeiro ideal de Fitting de $F_{\times} \mathcal{O}_{S(f)}$ é o ideal gerado pelos menores de ordem 3 de $\Lambda$. 'Iambém com o auxílio do Maple, calculamos a equação de $\Delta_{\text {sing }}=$ $V\left(\mathcal{F}_{1}\left(F_{*} \mathcal{O}_{S(F)}\right)\right)$, donde segue a equação de $B=\pi\left(\Delta_{\text {sing }}\right)$ e, portanto, a equação de $\left.B_{i}\right)$ :

$$
\frac{16}{25} u_{1}^{6}-\frac{224}{25} u_{1}^{4} u_{3}+\frac{1040}{25} u_{1}^{2} u_{3}^{2}-64 u_{3}^{3}+\frac{88}{25} u_{1}^{3} u_{2}^{2} \quad \frac{6336}{125} u_{1} u_{2}^{2} u_{3}-\frac{27}{5} u_{2}^{2}=0
$$

Suponhamos que $f$ seja como em (4.11). Segue de |11] que $f$ tem uma cúspide em $(x, y)$ se e somente se,

$$
\frac{\partial g_{a f}(x)}{\partial y}(y) \quad \frac{\partial^{2} g_{a_{f}(x)}}{\partial y^{2}}(y)=0 \quad \text { o } \quad \frac{\partial^{3} g_{u_{f}(x)}}{\partial y^{3}}(y) \neq 0
$$

Mas isto acontece se, o somente se, $y$ é uma singularidade do tipo $A_{2}$ de $g_{a_{f}(x)}$. Ainda, se $f$ tem uma dobra dupla em $\left(x, y_{1}\right)$ e $\left(x, y_{2}\right)$ entäo

$$
\begin{aligned}
& \frac{\partial g_{a_{f}(x)}}{\partial y}\left(y_{1}\right)=0 \quad \text { e } \quad \stackrel{\partial^{2} g_{a_{f}(x)}\left(y_{1}\right) \neq 0}{\partial y^{2}} \\
& \frac{\partial g_{u_{f}(x)}}{\partial y}\left(y_{2}\right)=0 \quad \text { o } \quad \frac{\partial^{2} g_{\alpha_{f}(x)}}{\partial y^{2}}\left(y_{2}\right) \neq 0
\end{aligned}
$$

ou seja, $y_{1}$ e $y_{2}$ são singularidades do tipo $A_{1}$ de $g_{a_{f}(x)}$ e $g_{a_{f}(x)}\left(y_{1}\right)=g_{a_{f}(x)}\left(y_{2}\right)$.

Reciprocamente, se $y_{1}$ e $y_{2}$ são singularidades do tipo $A_{1}$ de $g_{a_{f}(x)}$ e $g_{a_{f}(x)}\left(y_{1}\right)=$ $g_{a f}(x)\left(y_{2}\right)$, então cada ramo do bigeme de $f$ em $\left\{\left(x, y_{1}\right),\left(x, y_{2}\right)\right\}$ é man dobra. logo, $f$ tem uma dobra dupla em $\left\{\left(x, y_{1}\right),\left(x, y_{2}\right)\right\}$ se, o somente se, $y_{1}$ e $y_{2}$ são singularidades do 
tipo $A_{1}$ de $y_{a_{f}(x)}$ e $g_{a_{f}(x)}\left(y_{1}\right)=g_{a_{f}(x)}\left(y_{2}\right)$.

No primeiro caso $a_{f}(x) \in B_{C}$ e no segundo $a_{f}(x) \in B_{D}$. Segue daí que se a concontra $B$ somente em seus pontos regulares e. nestes pontos, transversalmente, entâo $f$ é estável o neste caso

$$
\begin{aligned}
& c(f)=\text { número de vezes que } a_{f} \text { encontra } B_{C} \\
& d(f)=\text { número de vezes que } a_{f} \text { encontra } B_{I}
\end{aligned}
$$

Dizemos que um subconjunto do $\mathbb{C}^{n}$ a um conjunto algábrico se satisfaz um número finito de equaçòes polinomiais. Segue que um subconjunto algébrico de Cé mma mião finita de pontos.

Teorema 4.3.3 Se fe como en (4.11) enläo

$$
c(f)=v\left(b_{c} \circ a_{f}\right) \quad d(f)=v\left(b_{D} \circ a_{f}\right)
$$

onde $b_{C}$ e $b_{D}$ são as equaços reduzidas de $B_{C}$ e $B_{D}$ respectivamente, e vé a ordem de $0 \in \mathbb{C}$.

Demonstração Numa demonstração análoga à do 'leorema 4.3.1 vemos que, a menos de mudança cle coordeniadas, qualquer desdobramento de fé induzido por um desdobramento do germe $a_{f}:(\mathbb{C}, 0) \rightarrow\left(\mathbb{C}^{k}{ }^{1}, 0\right)$ o vice-versia.

Suponhamos que exista uma deformação $a_{t}$ a 1-parâmetro da curva $a_{f}$ tal que, para $t \neq 0, a_{\ell}$ encontra $B_{C}$ o $B_{D}$ somente em seus pontos regulares e, nestes pontos, ransversalmente. Então a deformação $f_{t}$ induzida de $a_{t}$ é tal que $f_{t}$ e estável para $t \neq 0$, 0 número de cúspides no discriminante de $f_{l}$ é igual ao número de zeros de $b_{c}$ o $a_{t}$ e cada um destes zeros é simples. Temos que localmente o múmero de zeros de uma função $\mathbb{C} \rightarrow \mathbb{C}$ é preservado por deformaçũo. Logo

$$
v\left(b_{C} \circ a_{t}\right)=v\left(b_{C} \circ a_{f}\right)
$$

para todo t. Portanto o número de cúspides no discriminante de $f_{t}$ e $v\left(b_{c}\right.$ o a $\left.a_{f}\right)$. Analogatmente, o número de dobras cluplas no discriminante de $f_{l}$ é $v\left(b_{b} \circ a_{f}\right)$.

Resta mostrar que existem deformações a 1-parámetro de af com tais propriedades. Seja $b=b_{C} b_{D}$. Vamos primeiramente obter $a_{t}$ de forma que $b$ o $a_{t}$ tenha $v\left(b \circ a_{f}\right)$ zeros simples. Tomemos $\tilde{a}:(\mathbb{C}, 0) \rightarrow\left(\mathbb{C}^{k-1}, 0\right), \widetilde{a}(x)=\left(\widetilde{a}_{1}(x), \ldots, \widetilde{a}_{k} 1(x)\right)$ uma curva transversal a $B$ e definimos

$$
a_{l}(x)=a_{f}(x)+l\left(\tilde{a}(x)-a_{f}(x)\right)
$$

Considerando $b$ o $a_{t}$ como um polinomio em $t$, o conjunto dos valores de t para os quais $b$ o $a_{t}$ tem rázes repetidas á algébrico. Tal conjunto não é todo $\mathbb{C}$, pois $b$ o $a_{1}=b \circ \tilde{a}$ 
tem somente raízes simples. Logo este conjunto algébrico é um número finito de pontos e, portanto, $t=0$ é um ponto isoliado deste conjunto. Assim, para $t \neq 0$ suficientemente peculeno, bo $a_{t}$ tem somente raízes simples.

'Temos gue

$$
\left.b \circ a_{t}=\left(b_{C} \circ a_{t}\right)\left(b_{1}\right) \circ a_{t}\right)
$$

Assim, $r$ é uma raiz de $b o a_{t}$ se, e somente se, $r$ é una raiz de $b_{t}$ o $a_{t}$ ou mma raiz de $b_{1} \circ a_{t}$. Como $b \circ a_{t}$ só tem raízes simples, entäo $b_{c} \circ a_{t}$ e $b_{l}$ o $a_{t}$ só tèm rázes simples. Isto significa que $a_{1}$ encontra $B_{C}$ e $B_{D}$ apenas em pontos regulares, o lá transversalmente.

Este resultado mostra que $c(f)$ e $d(f)$ são determinados pela interseçäo de $a_{f}$ com $B_{C}$ c $B_{1}$.

Apresentamos a seguir alguns exemplos para ilustrar a utilização do Teorema 4.3.3 no cálculo de $c$ e $d$.

Exemplos 4.3.4 1. Consideremos $\int_{1, k}:\left(\mathbb{C}^{2}, 0\right) \rightarrow\left(\mathbb{C}^{2}, 0\right)$ dada por $f_{1 k k}(x, y)=\left(x, y^{4}:\right.$ $\left.x^{k} y\right)$. Temos que $m\left(f_{1, k}\right)=4$ e $a_{f, k}(x)=\left(0, x^{k}\right)$. Então

$$
\begin{gathered}
b_{C}=8 u_{1}^{3}+27 u_{2}^{2} \text { ○ } b_{C} \circ a_{f \ldots k}(x)=27 x^{2 k} \\
b_{D)}=u_{2} \circ b_{1)} \circ a_{f_{1, k}}(x)=x^{k}
\end{gathered}
$$

Portanto

$$
\begin{aligned}
& c\left(f_{1, k}\right)=v\left(b_{C} \circ a_{f_{1, k}}\right)=2 k \\
& d\left(f_{1, k}\right)=v\left(b_{\nu} \circ a_{f_{1, k}}\right)=k
\end{aligned}
$$

2. Seja $f_{2, k_{1}, k_{2}}:\left(\mathbb{C}^{2}, 0\right) \rightarrow\left(\mathbb{C}^{2}, 0\right)$ dada por $f_{2, k_{2}, k_{2}}(x, y)=\left(x, y^{4}+x^{k_{1}} y+x^{k_{2}} y^{2}\right)$, onde $3 k_{2}<2 k_{1}$. Ternos que $m\left(f_{2, k_{1}, k_{2}}\right)=1 c a_{f_{2, k_{1}, k_{2}}}(x)=\left(x^{k_{2}}, x^{k_{1}}\right)$. Lintàu

$$
\begin{gathered}
b_{C}=8 u_{1}^{3}+27 u_{2}^{2} \text { e } b_{C} \circ a_{f_{2, k_{1}, k_{2}}}(x)=8 x^{3 k_{2}}+27 x^{2 k_{1}}=x^{3 k_{2}}\left(8+27 x^{2 k_{1}-3 k k_{2}}\right) \\
b_{D}=u_{2} \circ b_{D} \circ a_{f_{2, k_{1}, k_{2}}}(x)=x^{k_{1}}
\end{gathered}
$$

Segue que

$$
\begin{aligned}
& c\left(f_{2, k_{1}, k_{2}}\right)=v\left(b_{C} \circ a_{f_{2, k_{1}, k_{2}}}\right)=3 k_{2} \\
& d\left(f_{2, k_{1}, k_{2}}\right)=v\left(b_{D} \circ a_{f_{2, k_{1}, k_{2}}}\right)=k_{1}
\end{aligned}
$$

3. Lomemos $f_{3, k_{1}, k_{2}}:\left(\mathbb{C}^{2}, 0\right) \rightarrow\left(\mathbb{C}^{2}, 0\right)$ dada por $f_{3, k_{1}, k_{2}}(x, y)=\left(x, y^{4}+8 x^{3 k_{1}} y-\right.$ $\left.6 x^{2 k_{1}, y^{2}}+x^{2 k_{1}-k_{2}} y^{2}\right)$ com $k_{2}>0$. Temos que $m\left(f_{3, k_{1}, k_{2}}\right)-4$ e $a_{f_{3, k_{1}, k_{2}}}(x)=\left(6 x^{2 k_{1}}-\right.$ 
$\left.x^{2 k_{1}+k_{2}}: 8 x^{3 k_{1}}\right)$. Entäo

$$
\begin{gathered}
b_{C} \circ a_{j_{3, k_{1}, k_{2}}}(x) \quad 8 x^{6 k_{1}+3 k_{2}}-144 x^{6 k_{1}+2 k_{2}}+864 x^{6 k_{1}+k_{2}} \\
b_{D} \circ a_{j_{3, k_{1}, k_{2}}}(x)=8 x^{3 k_{1}}
\end{gathered}
$$

Segue que

$$
\begin{gathered}
r\left(f_{3, k_{1}, k_{2}}\right)=v\left(b_{c} \circ \circ a_{f_{3, k_{1}, k_{2}}}\right)=6 k_{1}+k_{2} \\
d\left(f_{3, k_{1}, k_{2}}\right)=v\left(b_{1} \circ a_{f_{3, k_{1}, k_{2}}}\right)=3 k_{1}
\end{gathered}
$$

Pelo Corolário 4.2.11 temos os germes acinla são topologicamente distintos, já que os números ce el sio dois a clois distintos. 


\section{Referências Bibliográficas}

11 M. F. Atiyah e I. G. Macdonald, Introduction to comutative algebra, Addison-Wesley Publishing Company, 1969.

|2| W. Bruns and J. Her\%og, Cohen-Macaulay rings, Cambridge Lniversity Press, London, 1993.

|3| D. Eisenbud, Commutative algebra with a view toward algebraic geometry, Springer, New York, 1995.

44 T'. Fukuda and G. Ishikawa, On the number of cusps of stable perturbations of a plane-to-plane singularity, Tokio J. Math. 10 (1987 375-384).

[5] T. Gaffney, Propierties of finitely delemined germs, Thesis, Brandeis University, 1975.

16| T. Gaffney, Polar multiplicities and equisingularity of map germs, Topology, 32, 1993, $185-22: 3$.

(7) T. Gaffney and A. Du Plessis and L. Wilson, Map-germs determined by their discriminants, stratifications, singularitios and differential equations, I, Travaux en Cours, 54 (1997), 1-40, Hermann.

18| T. Gaffney and D. M. Q. Mond. Cusp and double folds of germs of analitic maps $\mathbb{C}^{2} \rightarrow \mathbb{C}^{2}$ : London Math. Soc. (2) 43 (1991) 185-192.

|9| T. Gaffney and D. Mond, Weighted homogeneous maps from the plane to the plane, Math. Proc. Camb. Phil. Soc. 109 (3), 1991, 451-470.

[10] C.G. Gibson, Singular points of smooth mappings, Research Notes in Maths., 25, Pitman, 1979.

111| M. Golubitsky and V. Guillonin, Stable mappings and their singularities, GlM 14, Springer-Verlag; 1973. 
|12| R. C. Gunning, Lectures on complex analytic varieties: the local parametrization theorem, Lniversity Press, Princeton, 1970.

[13] R. C. Gunning, Lecturss on complex analytic varieties: finite analytic mappings, Liniversity Press, Princeton, 1974.

|14| R. C. Gumning and H. Rossi, Analytic functions of several complex vorables, PrenticeHall, EUA, 1965.

|1j| R. Hartshome, Algebraic geometry, Fourth Printing, Springer-Verlag, New York, 1987.

|16| E. X. Looijenga, Isolated singular points on complete intersections, LMS Lecture Notes 77, Tondon, Cambridge University Press, 1984.

|17| S. Max Lane, Homology, Springer, New York, 1995.

|18| J. Martinet, Singularities of smooth functions and mappings, L.MS Lecture Notes 58 , Cambridge Lniversity Press, 1982.

|19| II. Matsumura, Commutative algebra, W. A. Benjarnin, Inc, Sew York, 1970.

|20| H. Matsumura, Commutative ring theory. Cambridge University Press, 1986.

21| J. Milnor, Singular P'oints of Complex Hypersurfaces, Annals of Mathemalics Studies 61, University Press, Princeton, 1968.

|22| D. Mond, Singularities of mappings from surfaces to 3-space, Singularity Theory, D.T., Lê, K. Saito, B. Tessier. eds, World Scientific, Singapore (1995), 509-526.

[23| D. Mond and R. Pellikaan, Fitting ideals and multiple points of analytic mappings, Procedings of the Ptzcuaro Workshop on Algebraic and Analytic Geometry, Iecture Notes in Mathematics 1414 (Springer, Berlin, 1991).

|24| M. Reid, Undergraduate algebraic geometry, Cambridge University Press, London, 1988.

|20| J.H. Rieger, Fanilies of maps from the plane to the plane, J. London Math. Soce (2) 36 (1987), $351-369$.

$|26|$ I. 'lari, Singularidades de aplicaçoes diferenciaveis, Notas Didáticas do ICMC, 34, 1999. 
[27] B. Teissier, The Hunting of invariants in the geometry of the discriminant, Real and Complex Singularities (ed. P. Holn; Sijthoff e Noordhoff, Oslo, 1977)564-648.

|28| L. D. Tráng, Iopologie des singularités des hypersurfaces complexes, Singularités à Cargèse, Asterisque $7 / 8$ (Societé Wathemetique de France, Paris. 1973) 171-182.

[29] C. T. C. Wall, Finite determinancy of smooth map-germs, Bull. London Math. Soc. $13(1981) 481-539$.

|30| H. Whitney, () Singularities of mappings of Fuclidean spaces .I.Mappings of the plane into the plane, Ann. of Math. 62 (1955) 374-410. 


\section{Índice Remissivo}

h-jatos, 3

equivalentes, 4

anéis

de Cohen-Macaulay, 20

Noetherianos, 16, 17

reduzidos, 31

aplicacão tangente, 7

campo de vetores, 8

Cohen-Macaulay

ancéis, 20)

germes de variedades, 26

módulos, 20

curva, 31

reduzida, 32,33

decomposição

primária, $15,16,25$

delta, 33

desdobramento

versal, 10

desdobramentos, 10

induzidos, 10

isomorfos, 10

topologicamente triviais, 11

triviais, 10

dimensão

de germe de variedade, 26

de um anel, 17

de urn módulo, 18

finita, 18 espaco tangente, 7

estácel

germie, 9

fibrado tangente, 7

finitia

«plicação, 26

germes

$\mathcal{A}$-equivalentes, 2

$\mathcal{K}$-equivalentes, 13

de corank 1,47

de subvariedade analítica, 23

de subvariedades, 25

de variedades, 24

de variedades de Cohen-Macaulay, 26

de variedades equidinnensionais, 26

estáveis, 9,30

finitamente determinados, 9

nultigerme, 1

rank, 2

grade

de um módulo, 20

grupos

ação de, 3, 4

de Lie, 3, 4

Hadamard, 11

Ililbert-Burch, 21

idleal

altura de, 17

de Fitting, 29, 37 
primário, 15

primo, 15

primo maximal, 15

primo minimal, 15

radical, 15

interseção completa, 28

interseção completa com singularidade:

isolada (ICIS), 28

módulos

de Cohen-Macaulay, 20

Noetherianos, 17

projetivos, 21

Malgrange, 13

Milnor

fórmula de, 33

número de., 33

nilpotente, 15

Noetheriano

anel, 17

módulo, 17

normalização, 27,34

profundidade

de um módulo, 20

pull-back: 10

secquência

exata, 21,22

regular, 18

subvariedades analíticas, 23

germes de, 23

Weierstrass, 12 